\title{
Remotely actuated polymeric nanocomposites for biomedical applications
}

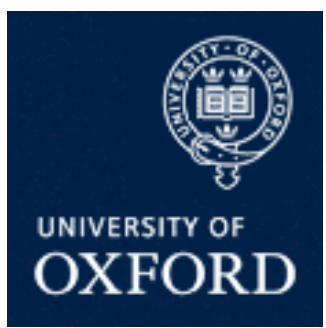

Ileana Andrea Bonilla Brunner

St. Edmund Hall

University of Oxford

Supervisor

Sonia Contera

A thesis submitted for the degree of

Doctor of Philosophy

Hilary 2018 


\begin{abstract}
The present work describes the synthesis, structural and mechanical characterisation of soft polymeric materials used for biomedical purposes.

This thesis is separated in two parts: the first one pays special attention to natural polymeric hydrogels for Tissue Engineering (TE) described in chapter 3 and chapter 4 describes the synthesis of magnetically responsive nanocomposite hydrogels for TE.

In this first part, I mainly focus on the mechanical characterisation of the synthesised materials. I use AFM microindentations to characterise them locally and oscillatory shear rheometry to characterise the bulk responses of the material, obtaining the reduced Young's modulus $\left(E^{*}\right)$, the storage $G^{\prime}$ and the loss $G^{\prime \prime}$ modulus respectively. Furthermore, in chapter 3 , the assessment of the stability of microbeads made of alginate for modular TE is described, followed by a novel magneto-mechanical coupling observed for the first time in chitosan hydrogels. In the 4 th chapter, I characterise the mechanical response of a chitosan- $\mathrm{Fe}_{3} \mathrm{O}_{4}$ nanocomposite with an enhanced magneto-mechanical coupling.

The second part of this work (chapter 5) describes a simple synthesis of polymeric micro-ellipsoidal particles for torque generation assays in biomedicine. I characterise the rotation of these particles in comparison to commercially available spherical microparticles and their enhanced ability to follow low magnetic rotating fields.
\end{abstract}




\section{Acknowledgements}

I would like to specially thank Dr. Sonia Contera for all of the help and guidance provided to me during the development of my DPhil project and the writing of this thesis and the papers. For believing in me and being so supportive in strengthening my weaknesses and enhancing my abilities.

From Contera's lab, I would like to thank Dr. Jacob Seifert for his endless help and support, for sharing with the group his amazing indentation curve fitting code and making our lives so much easier. For spearing time to read my whole thesis and guidance through couple of mock VIVAs as well as for all the patience while explaining AFM theory to a non-physicist like myself. Even more so for the amazing Dominoes nights, super-in-depth late-night confidence-uplifting talks, as well as for his friendship and in general for sharing his DPhil degree with me. Alba Piacenti for arriving to the Chitosan project in perfect timing to hate it with me but push it forward. For all the long AFM experimental days with countless cigarette breaks, cheer ups, emotional support and exceptional friendship. Casey Adams for her cheerfulness and interesting chats as well as to Dr. Zeinab Al-Rekabi for her support and advice and Armando for his company and friendship.

I would also like to acknowledge Thomas for all the work on the Alginate project, his company and hard work during AFM indentations and his friendship.

Furthermore, I would like to thank all the people that made this DPhil degree so memorable. To Alfonso for pushing me to work harder during thesis writing period, for the presentation practice provided and especially his company, and along with Francisco and Ninad for all the Dominoes and stress-free pub time. To Gaby for the company, advice, adventures and unconditional friendship. To Midori and Adán for their chemistry mastery advice, lunch break company and amazing friendship. To the whole Aston crew for an unforgettable first year. 
To Antonio Hurtado for his last minute latex help, interest in my work, getting me into theatre and impro and his unconditional and lovely friendship.

I am especially grateful with my family for all the love and unconditional support.

I am also very grateful with Dr. Julian Dye and Dr. Mónica Luna for examining my thesis and for all the input and great suggestions to improve it.

Finally, I would also like to thank the Mexican Science and Technology National Council (CONACYT), St. Edmund Hall, the E.P.A. Cephalosporin Fund and the Oxford University Vice Chancellor's fund for the funding received throughout my degree as well as to the European Cooperation in Science and Technology (COST) for the STSM Grant that allowed me to do the summer internship in ETH Zürich Switzerland, as well as Dr. Salvador Pané for receiving me in his lab at ETH. 


\section{Contents}

1 Introduction 1

1.1 Tissue Engineering and Regenerative medicine . . . . . . . . . . . . . . . . 2

$1.1 .1 \quad$ Biophysical aspects . . . . . . . . . . . . . . . . . . . . . 4

1.1.1.1 Biocompatibility . . . . . . . . . . . . . . 4

1.1.1.2 Importance of material design: mechanobiology . . . . . . 6

1.1.1.3 Requirements of material design. . . . . . . . . . . . . 10

1.1 .2 Biomaterials in TE . . . . . . . . . . . . . . . . . . . . 11

1.1.2.1 Natural polymeric materials: hydrogels . . . . . . . . . . . 11

$1.1 .2 .2 \quad$ Synthetic polymeric materials . . . . . . . . . . . . . 13

1.1.2.3 Stimuli responsive nano and composite materials . . . . . 13

1.1.3 Smart magnetically responsive nano- and composite materials . . . 15

1.1.3.1 Material magnetic response . . . . . . . . . . . . 16

$1.1 .4 \quad$ Strategies for TE . . . . . . . . . . . . . . . . . . . . . 18

1.1.4.1 3D polymeric scaffolds . . . . . . . . . . . . . 20

1.1.4.2 Modular TE . . . . . . . . . . . . . . . . . . 21

1.1.5 Mechanical properties of soft polymeric materials . . . . . . . . . 21

$1.1 .5 .1 \quad$ Elasticity and viscosity . . . . . . . . . . . . . . . 22

$1.1 .5 .2 \quad$ Viscoelasticity . . . . . . . . . . . . . . . 23

1.1 .5 .3 Poroelasticity . . . . . . . . . . . . . . . . . . 24

1.1.5.4 Bulk vs micrometer mechanical properties . . . . . . . . . 24

1.2 Magnetic actuators: microparticles for torque generation . . . . . . . . . . 26

$1.2 .1 \quad$ Torque in soft polymeric materials . . . . . . . . . . . . . . . . 26

$\begin{array}{lll}1.2 .2 & \text { Magnetic bead composites for torque generation at the micron scale } 27\end{array}$ 


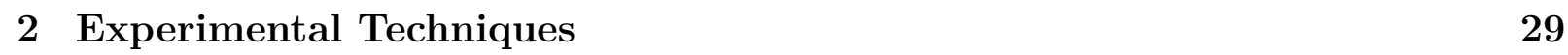

2.1 Abstract . . . . . . . . . . . . . . . . . . . . . . . . . . . . . . . . . . . . . . . . . . . . . . . . . 29

$2.2 \quad$ Atomic Force Microscopy $\ldots \ldots \ldots$. . . . . . . . . . . . . . . . 30

2.2 .1 AFM force measurements $\ldots \ldots \ldots \ldots \ldots$

2.2.1.1 Contact mechanics models: Hertz Model . . . . . . . . . 33

2.3 Oscillatory Shear Rheometry . . . . . . . . . . . . . . . . . . . . . . 35

2.4 Electron microscopy $\ldots \ldots \ldots \ldots$

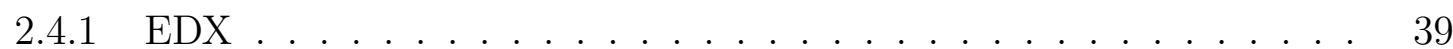

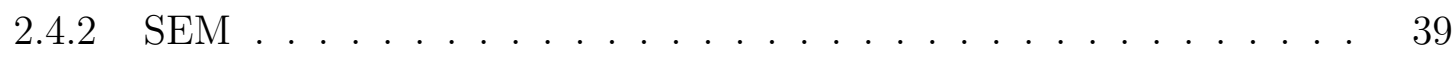

2.4 .3 TEM . . . . . . . . . . . . . . . . . . . . . . . 40

3 Mechanical properties of natural hydrogels for Tissue Engineering: Alginate and Chitosan 42

$3.1 \quad$ Alginate stability in modular Tissue Engineering applications. . . . . . . . 43 $3.1 .1 \quad$ Alginate $\ldots \ldots \ldots \ldots \ldots \ldots \ldots$

3.1 .2 Materials and methods . . . . . . . . . . . . . . . . . . 47

3.1.2.1 Alginate beads Preparation . . . . . . . . . . . . . 47

$3.1 .2 .2 \quad$ Assessment of Bead Swelling _ . . . . . . . . . . 47

$3.1 .2 .3 \quad$ AFM microindentation experiments. . . . . . . . . . . . 48

3.1 .3 Results . . . . . . . . . . . . . . . . . . . . . . . . 51

$3.1 .3 .1 \quad$ Bead Swelling . . . . . . . . . . . . . . . . . 51

$3.1 .3 .2 \quad \tilde{E}$ of alginate beads $\ldots \ldots \ldots \ldots$

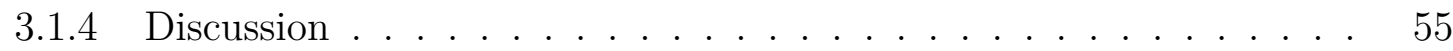

3.1.4.1 Bead Swelling . . . . . . . . . . . . . . . . . . . 55

3.1.4.2 Mechanical Properties measurement by AFM microinden-

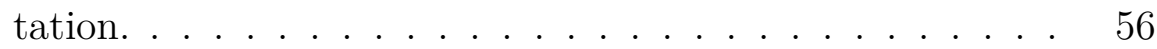

3.1.4.3 Hertz model . . . . . . . . . . . . . . . . . . . 56

3.1.4.4 Indentation experiments $\ldots \ldots \ldots \ldots \ldots$

3.1 .5 Conclusions . . . . . . . . . . . . . . . . . . . 58

3.2 Chitosan hydrogel mechano-magnetic coupling: An unexpected property . 60

$3.2 .1 \quad$ Introduction . . . . . . . . . . . . . . . . . . . . . 60

3.2.1.1 Chitosan . . . . . . . . . . . . . . . . 61

3.2.1.2 Chitosan Crosslinking . . . . . . . . . . . . . . . 62

3.2 .2 Materials and methods . . . . . . . . . . . . . . . . . . 64

3.2.2.1 Chitosan scaffolds fabrication . . . . . . . . . . . . 64 
3.2.2.2 Local mechanical characterisation: AFM-microindentations 65

$3.2 .2 .3 \quad$ Bulk Shear Rheometry mechanical characterisation . . . . 66

3.2 .3 Results . . . . . . . . . . . . . . . . . . . . . . . . . . . . . 67

3.2 .3 .1 AFM indentations . . . . . . . . . . . . . 67

3.2 .3 .2 Bulk shear rheometry . . . . . . . . . . . . . 67

3.2 .4 Discussion $\ldots \ldots \ldots \ldots \ldots$

3.2.4.1 AFM indentations . . . . . . . . . . . . . . 70

3.2 .4 .2 Bulk shear rheometry $\ldots \ldots \ldots \ldots \ldots$. . . . . . . 71

3.2.4.3 Magneto-mechanical coupling of hydrogels . . . . . . . 72

3.2 .5 Conclusions . . . . . . . . . . . . . . . . . . . . . . 73

\begin{tabular}{|lll}
\hline & Smart nanocomposite hydrogel for tissue engineering & 76
\end{tabular}

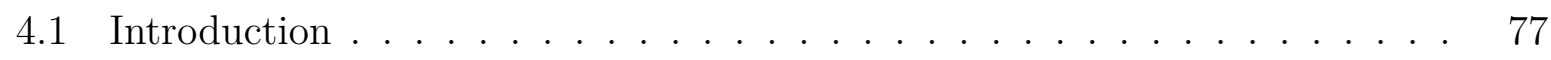

$4.1 .1 \mathrm{Fe}_{3} \mathrm{O}_{4}$ in biomedical research $\ldots \ldots \ldots \ldots \ldots \ldots \ldots$

4.2 Materials and methods . . . . . . . . . . . . . . . . . . . . . . . . 79

4.2 .1 Synthesis of $\mathrm{Fe}_{3} \mathrm{O}_{4}$ nanowires $\ldots \ldots \ldots \ldots \ldots$

4.2 .2 Chitosan scaffolds fabrication $\ldots \ldots \ldots \ldots \ldots$

4.2 .3 Structural characterisation . . . . . . . . . . . . . . . . . . . 81

4.2 .4 Local mechanical characterisation . . . . . . . . . . . . . . . . 82

4.2 .5 Bulk Shear Rheometry mechanical characterisation . . . . . . . . 82

4.3 Results $\ldots \ldots \ldots \ldots$. . . . . . . . . . . . . . . . . . . . . . . . 83

4.3 .1 Morphological characterisation . . . . . . . . . . . . . . . . 83

$4.3 .1 .1 \mathrm{Fe}_{3} \mathrm{O}_{4}$ Nanowires $\ldots \ldots \ldots \ldots \ldots \ldots$

4.3.1.2 Chitosan single component and chitosan nanocomposite morphological characterisation . . . . . . . . . . . . 83

4.3 .2 Mechano-magnetic scaffold characterisation. . . . . . . . . . 86

4.3.2.1 AFM microindentations . . . . . . . . . . . . . 86

$4.3 .2 .2 \quad$ Oscillatory Shear Rheometry $\ldots \ldots \ldots \ldots$

4.4 Discussion and conclusions . . . . . . . . . . . . . . . . . . . . . . . . . . . . 91

\begin{tabular}{|lll}
5 & High-speed rotating microparticles & 94
\end{tabular}

5.1 Introduction . . . . . . . . . . . . . . . . . . . . . . . . . . . . . . 95

$5.1 .1 \quad$ Magnetic shape anisotropy in colloidal composites . . . . . . . . . 97

5.2 Materials and methods . . . . . . . . . . . . . . . . . . . . . 98

$5.2 .1 \quad$ Synthesis of ellipsoidal microparticles $\ldots \ldots \ldots \ldots \ldots$ 
$5.2 .2 \quad$ Microparticle morphology and structural characterisation . . . . . . 99

$5.2 .3 \quad$ Microparticle rotational experiments . . . . . . . . . . . . . . . . . 99

$5.2 .3 .1 \quad$ Analysis of rotational experiments . . . . . . . . . . 101

5.3 Results and discussion $\ldots \ldots \ldots$. . . . . . . . . . . . . . . . . . 101

5.3 .1 Morphology and structural characterisation . . . . . . . . . . . . . 101

5.3 .2 Rotations per second calculation. . . . . . . . . . . . . . . . . 104

5.3 .3 Angular velocity calculation . . . . . . . . . . . . . 105

5.4 Conclusions . . . . . . . . . . . . . . . . . . . . . . . . 110

$\begin{array}{llr}6 & \text { Conclusions and further work } & 112\end{array}$

6.1 Conclusions . . . . . . . . . . . . . . . . . . . . . . . . . . . . . . . . . . . . 112

$6.1 .1 \quad$ Alginate microbead stability . . . . . . . . . . . . . . . . . . . 112

6.1 .2 Chitosan hydrogel and nanocomposite . . . . . . . . . . . . 113

6.1 .3 Microellipsoids . . . . . . . . . . . . . . . . . . 115

6.2 Further work $\ldots \ldots \ldots \ldots \ldots$

$6.2 .1 \mathrm{Fe}_{3} \mathrm{O}_{4}$ nanowires . . . . . . . . . . . . . . . 116

6.2 .2 Chitosan hydrogel scaffolds and nanocomposites . . . . . . . . . . . 118

6.2 .3 Microparticles . . . . . . . . . . . . . . . . . . 120

\begin{tabular}{|ll|}
\hline A Medium charge and Lorentz force & 121
\end{tabular}

\begin{tabular}{|l|l|}
\hline B Torque calculation for rotating microellipsoids & 127
\end{tabular}

B.0.1 Torque produced by weak magnetic fields . . . . . . . . . . . . . . . 129

\begin{tabular}{ll}
\hline Bibliography & 131
\end{tabular} 


\section{List of Figures}

1.1 Schematic representation of skeletal muscle crossbridge cycling contraction 8

1.2 Schematic representation of the orientation of the unpaired electrons spin in different magnetic responsive materials. . . . . . . . . . . . . . . . . . 17

2.1 Schematic representation of AFM contact mode . . . . . . . . . . . . . 31

2.2 AFM contact mechanics . . . . . . . . . . . . . . . . . . . . . . . . . . 33

2.3 Schematic representation of oscillatory strain deformation and shear rheometry set up . . . . . . . . . . . . . . . . 36

$2.4 \quad$ SEM high energetic electrons interacting with the sample . . . . . . . . . . 38

2.5 Schematic representation of SEM and TEM . . . . . . . . . . . . . . 40

3.1 Linear anionic copolymers in alginate . . . . . . . . . . . . . . . . . . 44

3.2 Changes in Alginate bead size and morphology under different conditions observed with optical microscopy. . . . . . . . . . . . . . . . . 52

$3.3 \quad$ Calculated $\tilde{E}$ of 2 different freshly made beads. . . . . . . . . . . . . . . . 53

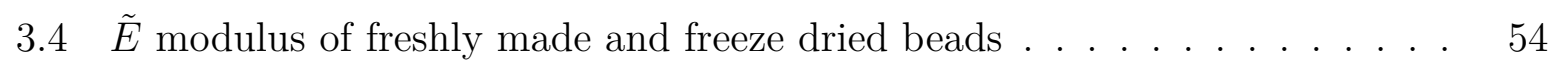

3.5 Chitosan to chitin deacetylation . . . . . . . . . . . . . . . . 61

$3.6 \quad$ Chitosan crosslinking with Genipin . . . . . . . . . . . . . . . . . . . . 63

3.7 Chitosan and PDMS AFM microindentations . . . . . . . . . . . . . . . . 68

3.8 Chitosan and PDMS bulk oscillatory shear rheometry . . . . . . . . . . . . 69

3.9 Magnetohydrodynamic effect in Chitosan . . . . . . . . . . . . . . . . . 74

$4.1 \quad$ Aligned nanowires embedded in chitosan . . . . . . . . . . . . . . . 77

4.2 Chitosan, chitosan $/ \mathrm{Fe}_{3} \mathrm{O}_{4}$ nanocomposite scaffold preparation process . . . 81

$4.3 \quad$ Nanowire AFM, TEM and SEM morphology characterisation . . . . . . . . 84

4.4 EDX analysis of synthesised nanowires . . . . . . . . . . . . . . . . 85

4.5 SEM of chitosan and chitosan composites . . . . . . . . . . . . . . 87

$4.6 \quad$ Chitosan nanocomposite polymers AFM microindentations . . . . . . . . . 89 
4.7 Bulk oscillatory shear rheometry on chitosan nanocomposites . . . . . . . . 90

5.1 Magnetic response of ellipsoidal colloids . . . . . . . . . . . . . . . . . . . . 98

5.2 Oil-in-water emulsion fabrication of the microellipsoids . . . . . . . . . . 100

5.3 SEM, TEM and EDX of microellipsoids . . . . . . . . . . . . . . . . . . 103

5.4 PCL only colloids . . . . . . . . . . . . . . . . . . . . . 104

5.5 Particle rotation angle and frequency $\ldots \ldots \ldots$. . . . . . . . . . 106

5.6 Rotations per second of microparticles . . . . . . . . . . . . . . . 107

108figure.caption.38

5.8 Coupled microparticles rotating counter-clockwise . . . . . . . . . . . . . . 110

110figure.caption.40

$6.1 \quad \mathrm{Fe}_{3} \mathrm{O}_{4}$ nanostructures embedded in PCL $\ldots \ldots \ldots \ldots$. . . . . . . . 117

B.1 Torque normalised by volume of particles and microellipsoids . . . . . . . . 130 


\section{List of abbreviations}

- AFM: Atomic Force Microscopy

- DMEM: Dulbecco's Modified Eagle's Medium

- ECM: extracellular matrix

- EDS: energy dispersive spectroscopy

- MPCs: Magnetic polymer composites

- NPs: nanoparticles

- PCL: Poly( $\varepsilon$-caprolactone)

- PDMS: polydimethylsiloxane

- PE: polystyrene bead

- PVA: poly(vinyl alcohol)

- SEM: Scanning Electron Microscopy

- TE: Tissue Engineering

- TEM: Transmission Electron Microscopy 


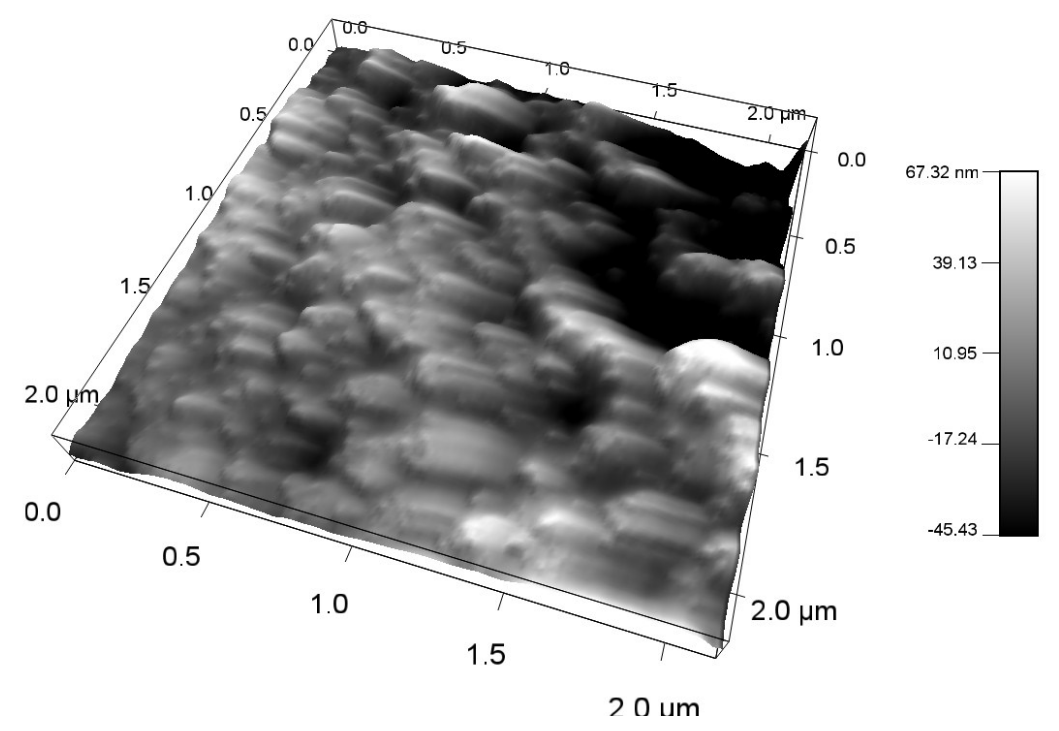




\section{Chapter 1}

\section{Introduction}

Polymers are the most functional class of materials used in biomedical applications due to their versatility of design and fabrication, the wide variety of structures and biomimetic properties, the possibility to tune them physically, chemically, mechanically and to functionalise.

This thesis describes some polymeric materials for biomedical applications, and its content can be divided in two parts: the first one focuses on natural and composite hydrogel scaffolds for Tissue Engineering (TE) and the second, on magnetically responsive nanocomposites as force generators at the micron scale.

In this introduction, I describe TE and the biophysical aspects to be considered in the design of polymeric scaffolds as well as the importance that the mechanical properties of the materials play in their use. I outline different kinds of biopolymeric materials used in TE, focusing on the natural hydrogels chitosan and alginate, as well as stimuli responsive nanocomposites, concentrating in magnetically actuated polymeric nanocomposite constructs used in TE and other biomedical applications.

I then briefly describe important responses to stresses on polymeric materials and experimental techniques to quantify their deformations and measure their mechanical properties. 
Lastly, I describe another use of magnetically responsive nanocomposites, this time in the form of microparticles which present enhanced capabilities to follow small rotating magnetic fields for force and torque generation assays.

\subsection{Tissue Engineering and Regenerative medicine}

Regenerative medicine is the field that pursuits to restore or establish normal function of human cells, tissues or organs by replacing, engineering or regenerating them [1]. Tissue Engineering (TE) is focused on developing tissue and organ substitutes by creating constructs that recapitulate the biological, biophysical and biochemical characteristics of real tissues.

Early experiments in 1970's that intended to generate new cartilage from chondrocytes seeded onto spicules of bone and implanted in nude mice [2], gave birth to the idea of TE that was only recognised until mid-1980's [3]. Since then, there have been great advances such as tissue-engineered autologous bladders for patients in the need of a cystoplasty [4] and the first synthetic trachea transplant made from a scaffold seeded with the patient's own stem cells in 2011 [5]. Furthermore, artificial skin and cartilage are examples of engineered tissues that have been approved by the Food and Drug Administration (FDA) [6].

Currently, the goal of TE is to assemble functional constructs that restore, maintain, or improve damaged tissues or whole organs for human implantation, animal free drug testing and conducting fundamental research to investigate basic biology of cells, tissues and organs including signalling pathways and disease.

In order to achieve this, it is essential to mimic the multi-scalar architecture of native tissues with features such as functional microvasculature, cell binding motifs, tissue specific morphology [7] and mechanical and structural stability that mimics the extracellular matrix 
(ECM).

The ECM is an heterogeneous, yet highly organised three-dimensional connective network of extracellular macromolecules secreted by cells. Different tissues have unique and specialised ECM components and organisation, as well as specific production and assembly, as well as temporal and spatial patterns to carry out tissue-specific roles. Several kinds of macromolecules compose the ECM, namely fibrous proteins (collagens and elastin), glycoprotein (fibronectin, proteoglycans and laminins) and enzymes. These macromolecules provide a physical scaffolding, mechanical stability, and biochemical cues necessary for tissue morphogenesis and cellular proliferation, migration and differentiation 8-11]. Glycoproteins are proteins to which carbohydrates are covalently linked through glycosidic bonds. Proteoglycans are a subclass of glycoproteins that contain glycosaminoglycan (GAG) components which are long, negatively charged, linear chains of disaccharide repeats that bind water to provide hydration and compressive resistance [8, 9]. Laminins are mosaic glycoproteins that mediate interactions between cells via cell surface receptors (such as integrins and dystroglycans). Similarly, many ECM proteins interact with cells through connections with fibronectin, which is a large multidomain glycoprotein that assembles via cell-mediated processes into fibrillar structures around cells [8]. Enzymes in the ECM are mostly metalloproteinases that are responsible for the constant remodelling and the dynamic structure of the ECM which is crucial for regulating morphogenesis [11]. Collagens are the most abundant proteins in the ECM and consist of three polypeptide $\alpha$-chains (homotrimers and heterotrimers), each of which contain amino acids bound together in repeating $(\mathrm{Gly}-\mathrm{X}-\mathrm{Y})_{n}$ sequences (where $\mathrm{X}$ and $\mathrm{Y}$ can be any amino acid) to form triple-helices that assemble into elongated fibrils $[8,9]$. Fibrillar collagen assembly involves multiple complex intracellular and extracellular post-translational steps that result in its unique mechanical properties and capacity to withstanding tensile forces 8 .

Dysregulation of ECM composition, structure and stiffness contributes to pathological 
conditions, such as fibrosis $[11,12$ and invasive cancer because of deregulation of normal behaviours of stromal cells that facilitate tumor-associated angiogenesis and inflammation 11, 13.

In addition to mimicking the mechanical and structural stability of the ECM, TE has other challenges to overcome in order to address the need of donor organs available for transplantation. In the biomaterial scope of the field, vascularisation of constructed tissue [14, 15] and mass transfer limitations in scaffolds [16] are a couple of the biggest challenges.

Vascular networks are necessary for cells to access to oxygen and nutrients as well as removing carbon dioxide and other cellular waste products during metabolism [14]. Angiogenesis, is the formation of new capillaries from pre-existing blood vessels and it is the primary mechanism for vascularisation of biomaterials. Appropriate internal structure of the scaffolds used such as pore size and pore interconnection have a strong impact on vascularisation and new blood vessel ingrowth $[16,17$.

In order to address these issues, TE biomaterial design has evolved from early stages when scaffolds were designed from bio-inert materials with appropriate mechanical and chemical properties to induce minimal biological adverse responses to second-generation resorbable biomaterials. These were designed to cause controlled cellular reactions and give space to the formation of ECM. Currently, third generation smart responsive materials are being developed to support and stimulate the regeneration of functional multicellular tissues $[18$.

\subsubsection{Biophysical aspects}

\subsubsection{Biocompatibility}

Biocompatibility is defined as the ability of a material to perform a function without eliciting any undesirable local or systemic effects [19]. 
There are different components to biocompatibility such as cytotoxicity, immunogenicity, genotoxicity/mutagenicity and carcinogenicity [20]. Cytotoxicity refers to damage of individual cells, in for example cell cultures. Immunogenicity refers to an immune response induced by a substance or material that often leads to inflammation. Genotoxicity describes the alteration of DNA base pair sequences which can lead to carcinogenicity, causing inappropriate cell growth into a tumour.

In the case of nanomaterials used in biomedical applications, biocompatibility is a crucial property that must be assessed. It is important to maximise nanostructure accumulation at target delivery sites which is limited by rapid blood clearance as well as nanomaterial internalisation by macrophages in filtration organs (i.e. liver, kidney spleen, and lung) which initiates inflammatory responses and induces toxicity 21.

Nanostructure internalisation by macrophages is highly dependent on particle size. Studies on internalisation of silica particles by mouse bone marrow-derived macrophages via an actin cytoskeleton-dependent pathway, show higher toxicological response (i.e. lysosomal destabilisation, cell death, and IL-1b secretion) of particles of $30-1000 \mathrm{~nm}$ than others up to $10 \mu \mathrm{m}[22]$.

In the same way, Karlsson et. al described a size dependent toxicology assay of $\mathrm{Fe}_{3} \mathrm{O}_{4}$ particles with diameters of $30 \mathrm{~nm}$ and $0.5 \mu \mathrm{m}$. They observed higher oxidative DNA damage by the $30 \mathrm{~nm}$ sized $\mathrm{Fe}_{3} \mathrm{O}_{4}$ particles at $80 \mu \mathrm{g} / \mathrm{ml}$, but no toxicity of either sizes at $40 \mu \mathrm{g} / \mathrm{ml}$. Similarly, at $80 \mu \mathrm{g} / \mathrm{ml}$, both $30 \mathrm{~nm}$ and $0.5 \mu \mathrm{m}$ sized particles showed mitochondrial depolarisation, suggesting a mitochondrial damage with subsequent cell death induced by apoptosis 23.

On the other hand, in vitro toxicity assays by Song et. al showed that Fe nanowires of $50 \mathrm{~nm}$ in diameter, internalised in HeLa cells, had no significant effect on the cell proliferation and viability even at high concentrations [24]. Similarly, Safi et. al studied the cytotoxicity of magnetic nanowires in NIH/3T3 mouse fibroblasts. The nanowires had 
diameters of $200 \mathrm{~nm}$ and lengths between 1 and $40 \mu \mathrm{m}$ and were fabricated by iron oxide $\left(\gamma-\mathrm{Fe}_{2} \mathrm{O}_{3}\right)$ nanoparticle assembly. Wires were internalised after $24 \mathrm{~h}$ and located either in membrane-bound compartments or dispersed in the cell's cytosol. Toxicity assays showed that the wires did not display acute short-term $(<100 \mathrm{~h})$ toxicity. Furthermore, cells were able to degrade the wires and to transform them into smaller aggregates [25].

Moreover, in vivo toxicity assays by of $\mathrm{Fe}_{3} \mathrm{O}_{4}$ nanoparticles (NPs) injected intravenously in mice show that NPs are mainly cleared from systemic circulation by macrophages residing in the reticuloendothelial organs. These NPs are presumably metabolised by macrophages in the lysosomal compartment via hydrolytical enzymes and proteins present in iron metabolism. The released free iron is gradually found as hemoglobin and partly bound to transferrin 26$]$.

\subsubsection{Importance of material design: mechanobiology}

The process of cellular differentiation is very complex. In addition to biochemicals such as growth factors, cytokines, and chemokines, cell-matrix interactions and mechanical stimuli influence cellular differentiation and tissue morphogenesis [27].

Mechanobiology studies the role of forces and mechanical properties in biological processes. Cells feel their environment mechanically (i.e. they are sensitive to stress or strain, such as compression, tension, or shear forces) as well as chemically, and respond to the mechanical stimuli by sensing physical forces and translating them into biochemical and biological responses in a process called mechanotransduction 28 31]. This process can direct cellular migration [32, cellular proliferation [33], angiogenesis [34], gene expression [35 38], cellular phenotype [39] and differentiation 40, 41].

This mechanosensing capacity of cells, allows them to actively probe the rigidity of their extracellular environment by exerting traction forces. At the molecular scale, matrix sensing requires the cell to anchor and pull against the matrix. Then, cellular mechano- 
transducer(s) generate signals based on the force that the cell applies to deform the matrix. Furthermore, a pathway of force transmission from inside the cell to the elastic matrix and vice versa is needed.

The anchoring and force transmission pathway is mainly formed by integrins which are the principal transmembrane receptors in animal cells, as well as other proteins such as cadherins, which are a type of cell adhesion transmembrane proteins important in the formation of adherens junctions to bind cells with each other, forming a tissue. Most integrins are connected to bundles of intracellular actin filaments which are part of the cell's cytoskeleton. Once ligand binding occurs, integrins activate signal transduction pathways that mediate cellular signals such as regulation of the cell cycle, organisation of the intracellular cytoskeleton, and movement of new receptors to the cell membrane [42]. Furthermore, after an initial integrin-ligand binding, the cytoplasmic tail of the integrin's $\beta$ subunit binds to other intracellular anchor proteins, including talin, $\alpha$-actinin, and filamin. These anchor proteins can bind directly to actin or to other anchor proteins such as vinculin, thereby linking the integrin to actin filaments in the cell cortex. This linkage leads to a clustering of the integrins and the formation of focal adhesions between the cell and the ECM 30 , $42,43]$.

Once these focal adhesions are formed, activated integrins can induce global cell responses such as changes in gene expression and intracellular signalling pathways. The signalling functions of integrins depend on a cytoplasmic protein tyrosine kinase called focal adhesion kinase (FAK) that is recruited to focal adhesions by intracellular anchor proteins such as talin. The clustered FAK molecules cross-phosphorylate each other, creating a phosphotyrosine docking site for a variety of intracellular signalling proteins which can then relay the signal into the cell. [42].

Once the cell has the path to relay information and anchors to pull from the matrix, cellular mechano-transducer(s) are needed to generate signals from the force needed by the 
cell to deform the matrix. Engler et. demonstrated that the nonmuscle myosin II is involved in the matrix-elasticity sensing that drives cell lineage specification. This cytoskeletal motors are implicated in tensioning cortical actin structures [40]. Myosins are a superfamily of motor proteins that convert chemical energy in the form of ATP to mechanical energy, thus generating force and movement.

Myosin motor proteins are involved in the process by which muscles contract which is describes by filament theory. It is a cycle of repetitive events known as 'crossbridge cycling' that cause a thin filament to slide over a thick filament and generate tension in the muscle [45].

A crossbridge is a myosin link as shown in figure 1.1, consisting of two myosin heads, that extends from the thick filaments (sarcomere). Each myosin head has with

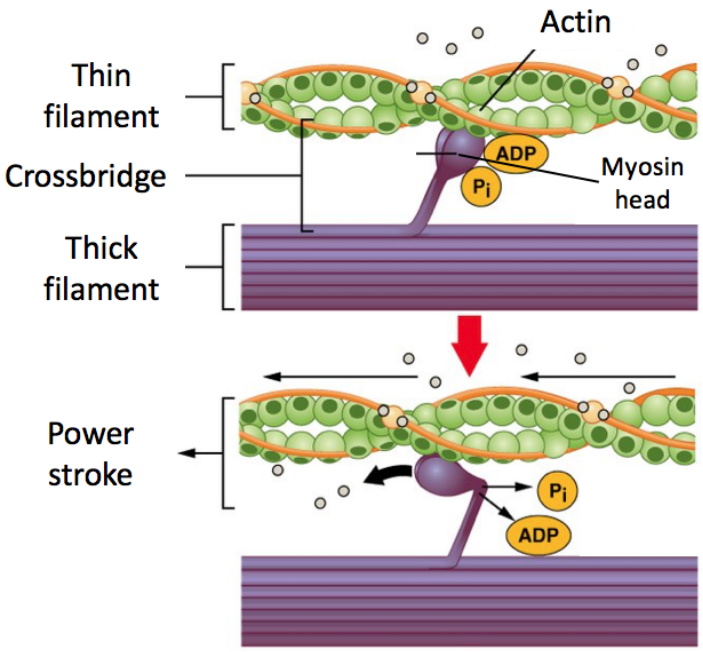

Figure 1.1: Schematic representation of skeletal muscle crossbridge cycling contraction. Modified from 44 . two binding sites, one for ATP and another for actin. The binding of ATP to a myosin head detaches myosin from actin, thereby allowing myosin to bind to another actin molecule. Once attached, the ATP is hydrolyzed by myosin, which releases the inorganic phosphate and initiates a power stroke, which generates a force of $2 \mathrm{pN}$. The power stroke moves the actin filament inwards, thereby shortening the sarcomere and moving along it [44].

Furthermore, optical tweezers studies by Helenius et. al of myosin-actin interactions show that a single myosin molecule is capable of exerting forces on the order of $\approx 3.5 \mathrm{pN}$ 46].

Actomyosin networks in all sorts of tissue, actively contribute to the cell's mechanical 
response to deformation, and their characteristic mechanical properties such as elasticity and viscoelasticity by conferring mechanical resistance to deformation and influencing cell and tissue stiffness. Elasticity occurs over short time scales where stretch or compression of actin networks lead to a restoration force proportional to the strain. When the strains occur over longer time scales, a viscoelastic response is observed due to assembly and disassembly of actin within the network and binding/unbinding of actin crosslinkers [47.

Actin proteins form filaments that are the smallest component with a diameter of $\approx 6 \mathrm{~nm}$ of the cytoskeleton which is a structure that helps cells maintain their shape and internal organisation, it also provides mechanical support that enables cells to carry out essential functions like division and movement. Actin networks that vary in network architecture can generate different types of force, such as protrusive and contractile force. Other components of the cytoskeleton are the microtubules which are the largest type of filament (diameter $\approx 25$ ) and are composed of protein called tubulin. Intermediate filaments are composed of different subunit proteins (diameter $\approx 10$ ).

The mechanosensing ability of cells confers a significant importance to the stiffness of the ECM, not only because of cellular differentiation processes, but also because of its influence on cellular phenotype and homeostasis. For example, Engler et. al 41 proved that myoblast, which are the primordial muscle cells that differentiate into striated or smooth muscle cells and form muscle fibers, present myosin/actin striations (striated tissue phenotype) within a very narrow range of normal muscle stiffness $(8-11 \mathrm{kPa})$. On glass and much softer or stiffer gels, differentiation is delayed, if not stopped. On very rigid substrates like glass, cells produce large isometric forces due to numerous vinculinenriched focal adhesions, limiting actin reorganisation into striations. On soft substrates, cell adhesion and contractile forces are extremely weak leading to poor spread of cells.

Moreover, Timraz et. al [39] showed that the phenotype and function of smooth muscle cells is modulated by stiffness of the substrate. They observed an increased secretion 
of collagen type I by these cells correlated to increasing surface stiffness. Increased levels of fibronectin and collagen type I, in combination with complex interactions between dysfunctional endothelium and inflammatory cells, lead to atherosclerotic lesions, hence correlating stiffness of the substrate with smooth tissue lesions.

Similarly, the stiffness of the ECM has been related to the modulation of cancer and influencing hallmarks of disease such as angiogenesis, migration, and metastasis [48 50] as well as the progression of diseases such as vascular disease, scar tissue formation, wound healing and fibrosis 51 .

This high sensitivity of cells to forces and substrate stiffness has been recognised and taken advantage of as a powerful tool in material design for TE. Tailored biomaterials can be tuned to mimic the physical and chemical properties of the ECM and interact with cells without adverse effects and to further direct tissue generation because of the stiffness influenced cellular phenotype $40,52,54]$.

\subsubsection{Requirements of material design}

Desirable characteristics of biomaterials used for TE are right surface chemistry to promote cell attachment, growth, migration and proliferation. Additionally, they must have high porosity to allow mass transport and diffusion of nutrients, oxygen, waste products and induce vascularisation [10].

Important issues arising from the use of highly porous materials are the resulting macro and microstructures that generate inhomogeneous polymeric lattices. Furthermore, it is essential to consider optimum pore size and porous continuity in the design to ensure cellcell communication and migration which can be inhibited if the pores are not interconnected [10]. More importantly, these properties have a huge impact on the local mechanical properties of the scaffolds, felt at a cellular level. This issue has been constantly neglected in the past given the big difficulty of fabricating homogeneous polymeric networks and will 
be further discussed in this thesis.

Additionally, the materials used in TE must provide a mechanical environment with adequate mechanical properties similar to the native matrix, i.e. toughness, rigidity, and elasticity that vary for different functions. They must also be mechanical and chemically biocompatible, to avoid immunologic responses and have bioactive cues and growth factors to regulate the cellular activities 10 .

Lastly, the role of the scaffold is temporary, thus, materials must have a biodegradability in a rate that closely matches regeneration of functional micro-vascular networks and remodelling of the desired tissue.

\subsubsection{Biomaterials in TE}

Some of the potential materials that have been extensively used in biomedical research include natural and synthetic polymers or a combination of the two, ceramic materials and composite materials. In this part of the thesis we will focus on natural and synthetic polymers for TE.

\subsubsection{Natural polymeric materials: hydrogels}

Natural materials are used to reproduce the chemical and physical environment of healthy tissues due to their biocompatibility, availability and the extensive knowledge on their chemistry and interactions as well as their mechanical properties.

Natural biomaterials such as collagens, glycosaminoglycan, nanocellulose, chitosan, alginate, elastin, etc. have been successfully used in TE [55, 56] and in repairing of nerves, skin, cartilage and bone 57], as well as in other biomedicine applications such as drug delivery [58, 59], antibacterial/antimicrobial agents [60], medical implants, wound healing [61], in diffusion driven biofactor and cell delivery [62], etc.

Hydrogels are hydrophilic polymer networks which may absorb up to thousands of times 
their dry weight in water $63[65]$. They can be chemically stable or degrade, disintegrate and dissolve. These polymeric networks are not homogeneous, since clusters of molecular entanglements or ionically bound domains can create inhomogeneities 65. Similarly, free chain ends are also present, introducing more network defects in these gels.

For the purpose of this thesis, I will focus on chitosan and alginate as materials for TE.

Chitosan is a biodegradable polysaccharide composed of D-glucosamine and N-acetylD-glucosamine subunits linked by a $\beta$ - $(1,4)$ glycosidic bond [66]. It is primarily obtained from the partial deacetylation of chitin present in the shells of shrimps which makes it inexpensive, biodegradable and biocompatible. It is also hypoallergenic, antibacterial, and bioadhesive. It is further described in chapter 3 as a hydrogel covalently crosslinked with genipin. Genipin is a natural crosslinker for proteins obtained from gardenia fruit extracts and commonly used in chitosan hydrogels fabrication.

Alginate is a natural polysaccharide derived from brown seaweed that consists of linear binary copolymers of a-D-mannuronic acid (M block) and b-L-guluronic acid (G block) residues in varying proportions, arranged in MM and GG blocks scattered with MG blocks 67]. Alginate is widely used as a biopolymeric hydrogel in biomedical research due to its capacity to be ionically crosslinked by divalent ions such as $\mathrm{Ca}^{2+}$ to form a gel under mild conditions without external heating or using strong organic chemicals 68 70].

Despite the extensive use of natural polymers in biomedical applications, poor mechanical performance has been a recurrent drawback. A way to counteract this is by reinforcing these materials [71] i.e. by incorporation of nanomaterials, which also opens the possibility of mimicking native ECM of biological soft tissues that consists of a fibre network with a complex multicomponent nano- and microstructure. 


\subsubsection{Synthetic polymeric materials}

Bioabsorbable polymers have been broadly used in biomedical applications, due to their biocompatibility and degradation capabilities by a simple hydrolysis process to products that can be metabolised by the body. Examples of these materials are poly-lactones such as poly-lactic acid (PLA) [72, poly(trimethylene carbonate) (PTMC), poly-glycolic acid (PGA) [73], and poly( $\epsilon$-caprolactone) (PCL) [74] and their copolymers.

In chapter 5 PCL is used in the form of composite microparticles in torque generation assays. PCL is a relatively inexpensive polyester suitable for modification which degradability, chemical and biological properties, physicochemical state and mechanical strength can be easily adjusted by the crosslinking ratio, crystallinity, molecular weight and blends with copolymers, hence useful under demanding mechanical, physical and chemical conditions without loss of its properties 75 .

PCL is obtained by ring-opening polymerisation of $\epsilon$-caprolactone monomers. These monomers polymerise via anionic, cationic, coordination and radical mechanisms.

PCL has been used in applications as micelles for the drug delivery [76], in the form of nanostructures for cancer treatment [77, 78], in bone [79, 80, cartilage [81] TE, as microparticles (described in section 1.2.2), etc.

\subsubsection{Stimuli responsive nano and composite materials}

Most smart biomaterials are commonly composite materials capable of changing their chemical structure, physicochemical properties, provide a mechanical or physical response, etc. as a response to one or more environmental variables (e.g., temperature, $\mathrm{pH}$, ionic concentration, light, electric and magnetic fields) (reviewed in 82).

Composite biomaterials have been extensively used in biomedical applications due to their higher mechanical strength, ability to tune mechanically and ability to be designed mimicking the native ECM microstructure. These composites are natural and synthetic 
polymers that have been reinforced with other polymeric synthetic fibers for connective tissue found in tendons, ligaments, fascia, bone and dermis [83, with natural fibers such as silk for cartilage TE 84,85$]$, bioceramic materials such as hydroxyapatite for bone regeneration [86], carbon nanotubes due to their very high aspect ratio and outstanding mechanical and electrical properties [87, nanostructures etc.

Drawbacks of reinforced materials are anisotropy, inhomogeneities and nonlinear properties with complex mechanical properties which make them hard to characterise mechanically.

Nanocomposite materials have been extensively used in $\mathrm{TE}$ in $\mathrm{pH}$ sensitive scaffolds [88], temperature responsive composites that facilitate patterned cell seeding [89], electric field responsive scaffolds for neural tissue regeneration [90] and magnetic field responsive composites for on-demand drug and cell delivery 91 .

Other biomedical applications of stimuli responsive nano and composite materials include the use of $\mathrm{pH}$, thermo- and magnetically responsive nanocontainers for targeted drug/gene delivery and hyperthermia [92, fabricated by 2-step shell polymerisation. Similarly, magnetic nanoparticles in polymer coated microparticles 93, 94, antibacterial composites [95], hyperthermia as cancer treatment [96], magnetically driven functionalised nanowire and nanoparticle sensors [97]. Furthermore, Yassine et al. [98 have utilised $\mathrm{Fe}_{3} \mathrm{O}_{4}$ nanowires to improve magnetic/thermally responsive $\mathrm{N}$-isopropylacrylamide (Nipaam) drug delivery systems, in comparison to previous NPs systems. They attribute their higher performance to the high remnant magnetization of the nanowires, which produce a larger torque when exposed to a magnetic field [99]. 


\subsubsection{Smart magnetically responsive nano- and composite mate- rials}

Magnetic fields $(\vec{B})$ are ideal external force generators because they are biologically inert and can be spatially and temporally controlled and can be safely used in aqueous environments.

Furthermore, magnetic fields deliver a better performance and higher forces in comparison to other manipulation strategies such as acoustic waves (including ultrasound), optical methods and electric fields 100$]$. Optical fields can only apply modest forces and require optical transparent environments and produce overheating 101 which limits their applicability. Acoustic waves rely on stiffness and density differences between the target matter and the environment but they tend to have relatively low and weak resolution 102 . Electric fields on the other hand, require a dielectric mismatch between the environment and the material, which allows them to deliver significantly more force than optical and acoustic methods. Nevertheless, they require local circuitry and large potentials that can lead to electrochemical reactions in aqueous and soft matter environments [100].

In TE, advantages of using magnetic fields have been reported, such as stimulation of proliferation of osteoblasts, promotion of growth factors expression, increment of osteointegration, enhancement of bone density and calcium content, stimulation of the recovery of bone fractures and accelerate new bone formation [103]. Zhang et. al demonstrated an increase bone density by a daily 30 min exposure for 30 days using an $8 \mathrm{~Hz}$ rotary $0.4 \mathrm{~T} \vec{B}$, suggesting potential applications in osteoporosis treatment 104 . Furthermore, molecular scale assays by Santini et. al used an oscillating $\vec{B}$ of $50 \mathrm{~Hz}, 0.5 \mathrm{mT}$ for one and two weeks to induce variations in the expression of cell adhesion molecules in two human osteosarcoma cell lines. They observed changes in cell growth and fibronectin and CD44 expression in MG-63 cells 105. 


\subsubsection{Material magnetic response}

A material has a magnetic behaviour when, if placed in a magnetic field of strength $\vec{H}$, the individual atomic moments in the material contribute to its overall response, the magnetic induction $(\vec{B}$, measured in Tesla $)$ is given by:

$$
\vec{B}=\mu_{0}(\vec{H}+\vec{M})
$$

where $\mu_{0}$ is the permeability of free space and $\vec{M}$ is the magnetisation $\vec{M}=m / V$ or magnetic moment $(\vec{m})$ per unit of volume $(\mathrm{V})$.

In magnetically actuated biomedical applications, the materials most commonly used are ferro-, ferri-, and paramagnetic or superparamagnetic. Their magnetic responses are shown in figure 1.2

Paramagnets are materials in which the spin of the unpaired electrons align to an applied $\vec{B}$. They do not retain magnetisation in the absence of the field, because the thermal energy randomises electron spin orientations.

Similarly, superparamagnetism is achieved in small enough (single-domain) magnetic particles/grains.For both paramagnets and superparamagnets in the absence of a magnetic field, the net magnetic moment $\vec{m}$ will average zero, whereas in the presence of a magnetic field, the total magnetisation at a particular temperature $(\mathrm{T})$ is given by the Langevin function:

$$
\vec{M}(t)=\operatorname{coth}\left(\frac{m H}{k T}\right)-\frac{k T}{m H}
$$

where $\mathrm{M}$ is the total magnetisation and $k$ is the Boltzmann constant. Despite that the magnetisation of a superparamagnet is similar to a paramagnet, the magnetic susceptibility is much larger than that of paramagnets.

In the case of superparamagnetism, $\vec{m}$ is not the result of a single atom but the ag- 
gregated value of the individual magnetic moments of $>10^{5}$ atoms from a single giant magnetic moment from a single-domain particle. These materials have a fast response to external magnetic fields as well as negligible residual magnetism (remanence) and coercivity $(\vec{B}$ necessary to reduce the magnetisation of a material to zero) 106,107 .

In ferromagnetic materials, the magnetic moment of the electrons interact with each other forming magnetic domains of different orientations as shown in figure 1.2. These different domains orient in one direction given a strong enough $\vec{B}$ and hold magnetism $\vec{B}=0$.

On the other hand, the spins of electrons in ferrimagnetic materials have unequal opposing magnetic moments resulting in a net magnetic moment. These materials do not lose magnetism in the absence of $\vec{B}$.

In biomedicine, magnetically responsive polymeric materials are attractive in a wide range of applications including biosensing, drug delivery and regenerative medicine (reviewed by 108), artificial muscles [109, sensors [110, controllable membranes [111], micro-robots (reviewed by [112]) and micropumps 113, 114. More specifically, Thomas et. al have synthesised magnetically responsive alginate Janus particles for targeted mesenchymal stem cell and drug delivery applications using iron oxide nanoparticles 115. Similarly, Ahmstad et. al synthesised $\mathrm{Fe}_{3} \mathrm{O}_{4}$ nanoparticles/liposome based magnetically controlled membranes by locally heating the membrane to change permeability without other envi-

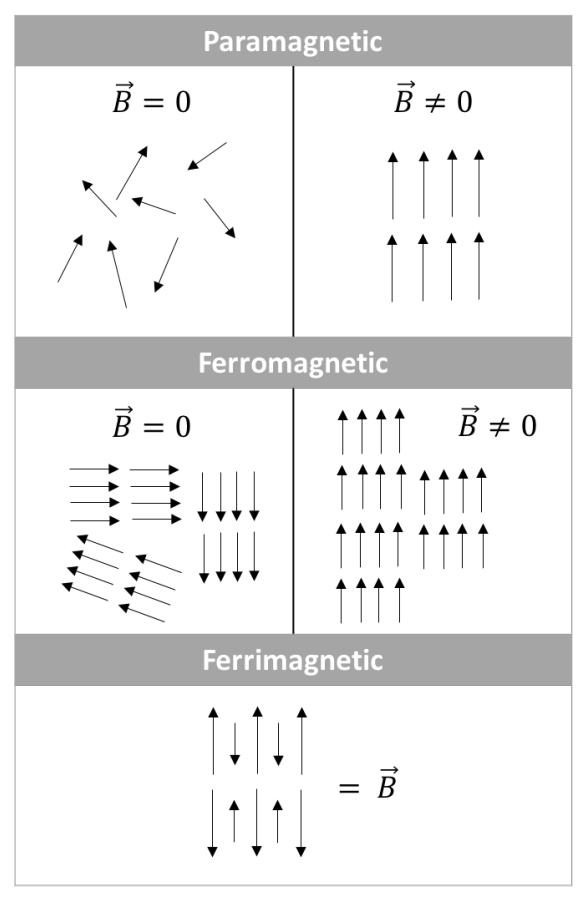

Figure 1.2: Schematic representation of the orientation of the unpaired electrons spin in different magnetic responsive materials. 
ronmental effects using alternating magnetic fields of $230 \mathrm{kHz}[116$.

Magnetically responsive biomaterials are the main subject in this thesis. In chapter 4 , I describe the fabrication and characterisation of a nanocomposite material composed of $\mathrm{Fe}_{3} \mathrm{O}_{4}$ nanowires embedded in a chitosan polymeric network. This composite is able to change its mechanical properties due to the presence of a $\vec{B}$.

In TE, in particular, magnetically controlling the mechanical properties of biomaterials used to seed cells in 3D cultures could direct cellular differentiation on demand by matching the stiffness of the scaffold due to a $\vec{B}$ to the desired native cell stiffness.

Furthermore, by controlling cellular responses, magnetically actuating the porous network could improve the diffusion of nutrients, drugs and growth factors and facilitate the growth of healthy tissue.

\subsubsection{Strategies for TE}

One of the main limitations in the fabrication of materials for TE is the controlled formation of pores, as mentioned previously. The size of the pore is essential and should be designed for matching cell size, since, if they are closed, too small or the relative density is too large, cells are not able to migrate through the scaffold [3, 47]. Macropororosity (pore size above $100 \mu \mathrm{m}$ ) is important for angiogenesis (process of creation of new blood vessels from pre-existing ones) and cell internalisation while microporosity can significantly enhance the specific surface area and improve the permeability of nutrients, oxygen, waste in scaffolds, thereby providing more protein adsorption sites and enhancing the degradation of scaffolds 117, 118. In the case of angiogenesis, studies have shown that the minimum pore size necessary for regeneration of a blood vessel is approximately $30 \mu \mathrm{m}$ to enable the exchange of metabolic components and to facilitate endothelial cell entrance. Yet, in order

to facilitate vascularisation of constructs, pores greater than $300 \mu \mathrm{m}$ are needed [118, 119]. In vitro, liquid applications must also consider pore size affecting intrinsic nutrient 
transport parameters like the diffusivity of solutes, the electrical mobility, and hindrance given hydrodynamic and steric interactions between solute molecules and the matrix molecules forming the pore walls $[3]$.

Furthermore, especial attention must be drawn on the effect of porosity on the mechanical properties. As porosity increases, density, strength, E and hardness decrease substantially, while surface area increases due to the large amount of void volume 3,47 , 120 .

Among the many techniques used to control porosity are electrospinning, solvent casting and particulate leaching, melt molding, membrane lamination, extrusion, freeze-drying, high-internal-phase emulsion, gas foaming, etc [3, 121].

Briefly, the solvent casting and particulate leaching technique involves casting a dissolved polymer around a porogen (such as sodium chloride) that will be leached out after drying and solidifying the polymer, yielding an interconnected porous network. A similar technique is melt molding where polymer and porogen particles are combined in a mold and heated above the glass transition temperature of an amorphous polymer or the melting temperature of a semicrystalline polymer. Then, the polymer is soaked to leach out the porogen. In the same way, the extrusion technique involves a mixture of a polymer and porogen forced through a nozzle by applying heat and pressure, followed by porogen leaching. An alternative method of fabrication is high-internal-phase emulsion, where the polymerisation of the a polymer dissolved in a continuous phase around aqueous droplets in a water-in-oil emulsion to obtain a solid network with interconnected pores. Or the gas foaming technique that involves compressing polymer disks and treating them with high-pressure $\mathrm{CO}_{2}$. When the pressure decreases, nucleation and pore formation occur obtaining an average pore size ranges from $100-500 \mu \mathrm{m}[3]$.

In this thesis I will focus on electrospraying and freeze-drying techniques. Electrospraying is used in chapter 3.1 to fabricate alginate beads for modular TE. This process involves 
3 main elements: a polymer source, a high voltage supply, and a collector. Briefly, this technique uses an electric potential applied between the positive electrode or the polymer source (normally a metal needle) and a collector (negative electrode). The needle accumulates a charge and forces (when the electric field overcomes the cohesive force and surface tension of the solution) a polymeric droplet to form and extrude into the collector plate. The polymer can then be deposited in the form of droplets or be elongated by electrostatic interactions between charges and form elongated fibres (process called electrospinning). In the mean time, the solvent evaporates and the polymer solidifies into a droplet. This technique permits control of the dimensions of the droplets or fibres for both, longitudinal and along the diameter in the case of electrospinning [122].

Freeze drying also known as lyophilisation or cryodessication; it is the process where low freezing point solvents are frozen into crystals which by a low vacuum level (between 50 mTorr and 100 mTorr) sublimate into a vapour phase due to the vacuum level set point being deeper than the vapour pressure of ice at the set temperature. This creates a cast from the solid solvent imprinted into the polymer. When the solvent sublimes, it leaves pores in the material. This process is widely employed in the food and pharmaceutical industry for long term preservation and storage and can extend shelf life and provide a pathway towards commercialisation [123]. This process is used in chapters 3 section 3.2 and 4 to fabricate materials for TE.

\subsubsection{3D polymeric scaffolds}

Traditional strategies for TE typically employ a 'top-down' approach, in which cells are seeded on a biodegradable polymeric scaffold [7]. The 3D scaffolds, depending on molecular weight of the material and crosslinking ratio, provide a mechanical strength that can be tuned to match a wide variety of native tissues [124].

In chapter 3, section 3.2 and 4 3D polymeric scaffolds composed of a chitosan poly- 
meric matrix and a composite with $\mathrm{Fe}_{3} \mathrm{O}_{4}$ nanowires were fabricated by freeze drying and mechanically characterised.

\subsubsection{Modular TE}

Modular TE is a bottom-up approach that comprised of repeating functional units designing structural features on the microscale, building from modules seeded with cells used as building blocks to recreate larger biomimetic tissues.

These modules aggregate into larger tissues through self-assembly, random packing, microfabrication of cell-laden hydrogels, stacking of layers, by creation of cell sheets or by directed assembly 7$]$.

This technique has been used in encapsulating pancreatic islet cells in alginate gels for transplantation in diabetic rats [125, embedded umbilical vein for smooth muscle tissue [126], etc.

In chapter 3 , section 3.1, I describe the fabrication, swelling and mechanical characterisation of alginate beads for stability assays in modular TE applications.

\subsubsection{Mechanical properties of soft polymeric materials}

The mechanical properties of polymers are dependent on many intrinsic characteristics such as the molecular weight, the density, the level of crystallinity (the structural order among polymeric chains, which results in less energy absorption during a deformation and higher strength than amorphous phases) and the crosslinking ratio. Increasing these parameters results in higher $\mathrm{E}$ [127, 128].

An appropriate design of polymeric materials for biomedical applications depends on mechanical reliability for implants as well as mechanical mimicking of native tissues and structures as the ECM in order to increase biocompatibility. 
Due to the viscoelastic nature of polymeric biomaterials, their mechanical characterisation is complicated since the polymer response to forces depends on both time and temperature. This dependency is correlated to macromolecular rearrangements and conformational changes as a result of a force driven deformation.

\subsubsection{Elasticity and viscosity}

Elasticity is the immediate return of solid materials to their original shape after forces are applied on them.

The elasticity of a material can be characterised by the stress $\sigma$ to strain $\varepsilon$ (relative deformation) relationship. Stress is a force acting on an unit area.

When the deformation is reversible and the stress-strain are linearly related, the material is behaving elastically. In a stress-strain curve, this elastic behaviour obeys Hooke's law:

$$
\sigma=E \varepsilon
$$

For a linear material, the stiffness is the slope of the stress-strain curve coincides with the Young's modulus $(E)$ which is an intrinsic property of the material.

Viscosity is a measure of a fluid's resistance to flow. Viscous materials deform under stress in a time dependent manner.

On the other hand, when there is a linear relationship between shear stress and shear rate, it obeys Newton's law of viscosity

$$
\sigma=\eta \dot{\gamma}
$$

where $\sigma$ is the shear stress and $\eta$ is the proportionality constant or the viscosity of the fluid. 


\subsubsection{Viscoelasticity}

Viscoelasticity is the property of materials that exhibit both viscous and elastic characteristics when undergoing deformation. The material recovers after the deformation but in a time-dependent manner. This results in a hysteresis due to energy dissipation.

As discussed in the previous section, the simplest models to describe deformation behaviour of ideal materials are for the solid state, Hookean linear elasticity and in the liquid state, Newtonian linear viscosity.

Viscoelasticity is the general case for polymers because their behaviour is highly timedependent and mostly nonlinear with a combination of elastic and viscous responses. The deformation and recovery are time-dependent, therefore frequency and deformation rate are relevant. The viscoelastic behaviour is dependent on temperature, deformation rate and structural variables such as the molecular mass, the crosslinking density (due to concentrations of polymer and crosslinker), crosslinking strength (given different kinds of bonds) and degree of crystallinity.

When a deformation results in a permanent change of the material, then it is a plastic deformation. In the case of polymers, this can be due to rupture of crosslinks between polymer chains.

In TE, the viscous response of the ECM significantly affects the behaviour and function of cells. [129]. The environmental viscoelasticity of the material has been shown to trigger molecular changes within migrating clusters of cells [130]. Furthermore, varying the viscoelastic properties of a polydimethylsiloxane (PDMS) substrate has modulated mesenchymal stem cells (MSC) expression of biomolecules such as osteopontin, a secreted phosphoprotein implicated in tissue repair and regeneration 131. 


\subsubsection{Poroelasticity}

Porous materials as the scaffolds described in this thesis used for TE behave poroelastically. As described previously, they are designed with high porosity to induce cell internalisation, mass transport, etc. This gives another degree of complexity to their mechanical characterisation, because an applied stress in an interconnected pore network pressurises the liquid medium.

Poroelasticity is also a time dependent behaviour introduced by the viscosity of the fluid that delays the flow through the pores $[132$ and hydrodynamic effects. However, a study by Wang et al. shows that at high compression speeds the time dependent behaviour is small enough to be neglected [133 and experimentally assessed by increasing the indentation speed until reaching a plateau in a measured $\tilde{E}$ modulus.

\subsubsection{Bulk vs micrometer mechanical properties}

As mentioned before, physiological processes are mostly guided by interactions that occur between cells and their local tissue environment. Measuring mechanical properties of materials at the micron scale is important since human cells dimensions are from $2-120 \mu \mathrm{m}$. Because of this, it is important to characterise materials used in TE from a local (at the nano- and micron scale) to a macroscopic range or in bulk. This is imperative, since the local measurements of inhomogeneous materials are not representative to the bulk behaviour even when the characteristic length of the inhomogeneity/mean porosity is an order of magnitude smaller than the indentation contact area 134.

$\mathrm{AFM}$ is a technique widely used to characterise the mechanical properties of materials at the nano- and micron scale by applying forces and quantifying the deformation responses of the materials 135, 136]. AFM has been successfully used to characterise scaffolds for TE such as soft scaffolds [137], 3D scaffolds for tissue-engineered cartilage constructs [138], of electrospun fibre scaffolds 139, 140, of porous scaffolds for bone TE 141, etc. 
In this thesis, this technique is used to mechanically characterise the local properties of the fabricated materials and in chapter 2 , theory behind microindentation experiments and Hertzian contact model to quantify the measured responses are described.

Additionally, oscillatory rheometry is a standard tool used to study the bulk structural and dynamic properties of materials by quantifying their bulk behaviour of viscous and elastic responses at different time scales and oscillation frequencies. Oscillatory shear rheometry has been used to characterise hydrogels [142] and nanocomposite materials for TE [143] as well as tissues such as auricular cartilage 144. 


\subsection{Magnetic actuators: microparticles for torque generation}

As described in section 1.1.3, magnetic field responsive nanocomposite materials have a wide range of applications due to the remarkable characteristics of magnetic fields as force generators in biomedical applications.

In this section and in chapter 5, another use of magnetic fields and nanocomposite materials are described, in the form of microparticle torque generators to measure forces at the micron scale.

\subsubsection{Torque in soft polymeric materials}

Generating and measuring forces at the micron scale in liquid and using biocompatible soft matter systems has become of interest in a large number of biological and medical applications such as studying cellular mechanotransduction [28, 145] microrheology of complex fluids, magnetic tweezers [146, 147], microrobotics [148, 149] and in the miniaturisation of clinical diagnostics systems.

Magnetically generated torque is used in soft matter for actuating, orienting or assembling systems and to develop new technologies and rheological experiments for the study of biological systems and life sciences [100]. There is a great interest in fabricating systems to produce torque since it can be used to generate rotation, in applications such as microfluidics 150, to assess liquid viscosity 151], magnetic tweezers [152, microrobotics 153 .

In order to perform complex tasks and movements such as controlled rotation it is necessary to exert torque on the particle. There are two ways of generating magnetic torque on materials possessing magnetic moment: (i) by using the magnetic anisotropy conferred by spin-orbit coupling, and (ii) by controlling shape anisotropy, which gives way to easy-axes 
of magnetisation such as the long axis in disks and rods. The demagnetisation processes of a material are highly dependent on the shape anisotropy 154 . In an applied $\vec{B}$, the most energetically favourable configuration is achieved when the easy axis of magnetisation (normally the long axis of a particle with shape anisotropy) aligns parallel with the direction of the applied field 154. The latter means that an internal magnetic torque that works to rotate anisotropic particles possessing magnetic moment towards the most energetically favourable configuration, can be generated [100, 154].

\subsubsection{Magnetic bead composites for torque generation at the mi- cron scale}

Magnetic beads have been preferred as a favourite design for small-scale mechanical technologies using soft materials due to the ease of fabrication, versatility and suitability for theoretical modelling.

Commercially available microparticles are fabricated generally as synthetic (mainly Polystyrene but also porous or non-porous silica) nanocomposites with superparamagnetic or ferromagnetic nanoparticles embedded inside the particle. This beads can be further superficially functionalised (with biotin, streptavidin, fluorescent dyes, hydroxyapatite, amino and carboxy groups, etc.) for many applications such as in the study of the Escherichia coli flagellar motor using magnetic tweezers 152,155 by attaching a bead to the hook of the flagellar motors to probe the motor's average speed, as well as reducing the average speed

of the motors until they stall, by increasing $\vec{B}$ strength from 1 to $20 \mathrm{mT} 155$. Similarly, biomagnetic separation is a technique frequently used for purification, sorting, etc. of cells, bacteria, DNA/RNA, proteins by specifically binding them to magnetic beads coated with antibodies or proteins with high affinity. The beads are then retained by a magnetic field while the supernatant is washed out 156 . 
Other applications such as microrheology applications 34, 157, lab-on-a-chip systems [158], drug delivery [98, 159, hyperthermia 160, 161] and microfluidics/mixing by applying a sinusoidal $\vec{B}$ of $30 \mathrm{mT}$ and a frequency of $20 \mathrm{~Hz}$, obtaining a mixing efficiency of $\approx 80 \%$ and an increment in efficiency to $95 \%$ by reducing the frequency to $5 \mathrm{~Hz} 150$.

In chapter 5, the fabrication of biocompatible nanocomposite microellipsoids with superior capabilities to follow magnetic fields than the commercially available counterparts are described. 


\section{Chapter 2}

\section{Experimental Techniques}

\subsection{Abstract}

In this thesis we describe the synthesis and mechanical characterisation of biopolymers used in tissue engineering in chapter 3, as well as the synthesis of magnetically responsive materials for tissue engineering in chapter 4 and nanocomposite microbeads for biomedical research in chapter 5 .

In this chapter we describe the theory behind the experimental techniques used throughout this thesis to characterise the local mechanical behaviour of the synthesised materials by AFM microinentations and the bulk material behaviour by means of oscillatory shear rheometry. We also characterise the morphology and structure of synthesised $\mathrm{Fe}_{3} \mathrm{O}_{4}$ nanowires and polymeric nanocomposites by AFM imaging, scanning electron micoscopy (SEM), transmission electron microscopy (TEM) and energy dispersive spectroscopy $(\mathrm{EDX})$. 


\subsection{Atomic Force Microscopy}

AFM is a microscopy technique from the Scanning Probe Microscopy family. In early 1980's, Gerd Binnig and Heinrich Rohrer, working in IBM developed the first Scanning Tunnelling Microscope (STM) for studying surface structure with atomic resolution. This microscope applies a voltage to the tip which creates a 'tunnel' of electrons jumping between the tip and sample. This flow of electrons generates a current that can be detected. They developed a feedback able to tune the distance between the tip and the sample by using piezoelectric ceramics to control the scanning movement and position. They received the Nobel prize in 1986 for their invention.

AFM works with a similar principle but relies on the interaction forces of the tip with the sample which lead to the deflection of the cantilever according to Hooke's law $F=\kappa d$ that linearly relates an applied force $(F)$ with the stiffness $(\kappa)$ of the cantilever.

Figure 2.1 shows the working principle of the simplest mode of this technique called 'contact mode', when the feedback is set on the deflection. A sample is scanned by a tip, mounted to a cantilever while, the motion of the deflection of the cantilever as a result of the interaction between the tip and the sample is monitored by the position of a laser reflecting on the back of the cantilever into a split photodiode. This signal is transformed into an electrical current sensitive to the deflection of the cantilever given the position of the reflected laser beam in each of the quadrants of the photodiode.

Then, the $\mathrm{z}$ or height position is monitored and controlled by a feedback loop. This feedback works by adjusting a voltage applied to a lead zirconium titanate (PZT) piezoelectric material (piezo) that expands and contracts given an electric field voltage. The voltage is applied in order to keep the deflection as a constant set value via a PID controller. The change of voltage relative to the real distance is calibrated beforehand. Finally, the $\mathrm{x}, \mathrm{y}$ and $\mathrm{z}$ positions of the piezo materials are correlated to form an image. 


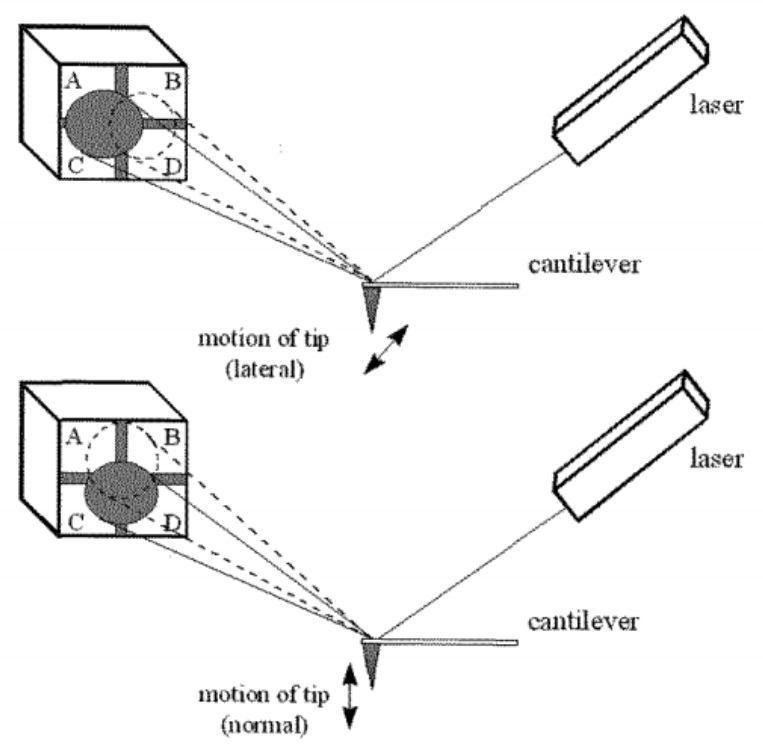

Figure 2.1: Schematic representation of the simplest mode of operation of AFM. Taken from 162 .

AFM has several operation modes that can have feedback in different parameters: (1) contact mode, feedback in deflection or lateral deflection, (2) tapping mode, with feedback on the amplitude (amplitude modulation or AC), the frequency (FM or frequency modulation) or the phase and (3) multifrequency AFM that uses two or more harmonics or eigenmodes of the cantilever at the same time.

\subsubsection{AFM force measurements}

In an AFM force measurement the tip attached to a cantilever is moved towards the sample while the deflection of the tip $\left(Z_{c}\right)$ and the position of the piezo $\left(Z_{p}\right)$ normal to the surface are recorded and converted into force and distance. The force $\mathrm{F}$ is obtained by multiplying the deflection of the cantilever with the spring constant $\left(k_{c}\right): F=k_{c} Z c$. The distance between the tip and the sample $(\delta)$ is calculated by adding the deflection to the position of the piezo: $\delta=Z p-Z c$. The obtained graph is called a force-versus-indentation curve or 'force curve'. 
Figure 2.2 (b) shows a force curve on an alginate bead for modular tissue engineering as further described in chapter 3 . The contact was made by a $20 \mu \mathrm{m}$ in diameter polystyrene bead (PE) attached to the cantilever in a liquid environment. Fast indentations were performed at $45 \mathrm{\mu m} \mathrm{s}^{-1}$. We can observe characteristic traces in this force curve as the loading (red) and unloading (blue) curves indicating the cantilever approach and retraction from the sample respectively.

In the non contact regime, the tip is far away from the sample until deflection of the cantilever is measured, indicating the first contact of it with the sample (known as 'contact point' and indicated with a black arrow).

Once this first contact happens, the curves are in the contact regime. The maximum indentation depth $\left(\delta_{\max }\right)$ marked with number 1 , is given by a trigger point which indicates the maximum force that is going to be applied by the system or a maximum deflection of the cantilever.

After this maximum indentation is reached, the tip starts retracting from the sample. Number 2 illustrates the hysteresis in the approach/retract curves because of viscoelastic responses of the sample 163,164 and energy dissipation that can be measured from this cycle. Although this effect is also partly due to the tip sliding on the sample when it gets in contact with the sample and the piezoelectric translator keeps approaching [165, 166].

Number 3 shows adhesion forces between the cantilever PE bead and the sample resulting from attractive forces such as capillary forces, chemical bonding forces and electrostatic forces. Once these adhesion forces are overcame, the tip is away from the sample and the curve is in the non contact regime again. Negative forces are attractive forces that are usually adhesion forces in the unloading curve and van der Waals forces in the loading curve. 

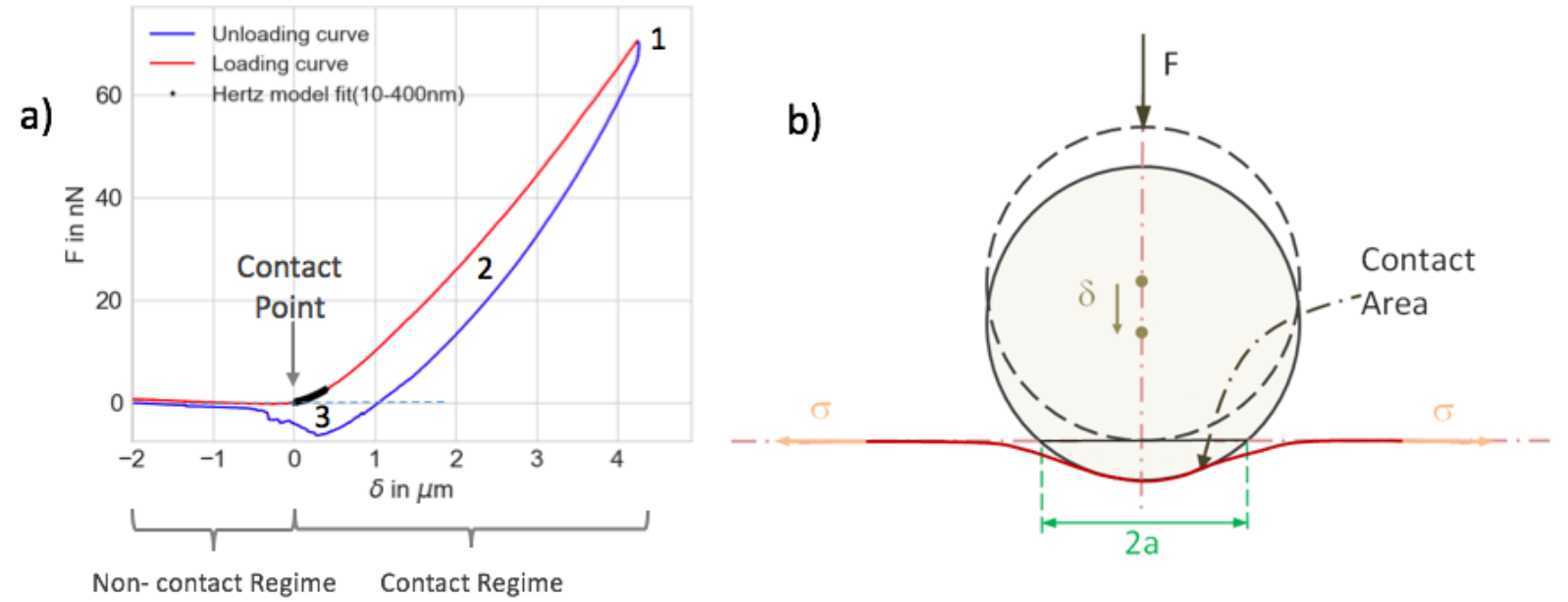

Figure 2.2: (a) Resultant force curve (force vs indentation $\delta$ ) measured in an alginate bead (chapter 3 ) showing the loading (red) and unloading (blue) curves with the contact point pointed by an arrow. Number 1) shows the maximum deflection of the cantilever given by a set trigger point; 2) the viscoelastic response hysteresis and 3) shows the adhesion region of the cantilever bead with the sample. (b) Schematic representation of a sphere indenting a plane. Taken from [167].

\subsubsection{Contact mechanics models: Hertz Model}

Contact mechanics studies the deformation of solids that touch each other at one or more points, considering different geometries and different contact angles.

Heinrich Hertz, in 1882 solved the contact mechanics problem of two elastic bodies with curved surfaces [168].

The classical solution for non-adhesive elastic contact between a sphere of radius $\mathrm{R}$ and a half-space is:

$$
F=g \tilde{E} \delta^{\alpha}
$$

where $\mathrm{F}$ is the force, $g$ and $\alpha$ are geometric-dependent factors and in the case of a sphere, $g=\frac{4}{3} \sqrt{R}$ and $\alpha=\frac{3}{2} ; \delta$ is the indentation and $\tilde{E}$ is the effective Young's Modulus, defined as: 


$$
\tilde{E}=\frac{E}{1-v^{2}}
$$

where $\nu$ is Poisson's ratio of the sample and $E$ is the Young's modulus.

Hertz model is simplest contact-mechanics model that assumes a hemispherical tip shape and that the applied strains are within the elastic limit since there is no consideration of energy dissipation during the interaction. It neglects surface interactions between the tip and the sample such as van der Waals, electrostatic and adhesion forces [169, 170], but can be applied if the adhesion forces are much smaller than the maximum load [166, 170].

Despite these limitations and given the lack of practicable nonlinear elastic contact models leads to relying on the Hertz model as an standard to analyse indentation data. Hertz model has been used in systems with very low surface energy (small adhesion forces) and small applied static loads (less than several nanonewtons) because wear and plastic deformation are prevented 170, 171. It has also been used to quantify the mechanical properties of tissues as cartilage [172] and stem cells [173].

Throughout this thesis, I restricted curve fitting to small indentations depths in the linear elastic regime, as well as small strains up much smaller that the $30 \%$ limit deformation [133, 174] to fall within Herzian limits. 


\subsection{Oscillatory Shear Rheometry}

Oscillatory rheometry is a technique that studies the structural and dynamic properties of materials by quantifying the bulk[1] or macroscopic mechanical behaviour of both, the viscous and elastic responses at different time scales.

This technique measures the resultant stress response when a sinusoidal shear deformation is induced in the sample at certain frequency of oscillation $\omega$.

We used a rheometer with a parallel plate geometry (Anton Paar Rheometer Physica MCR301) and a $7.966 \mathrm{~mm}$ plate diameter. In this setup, the sample is placed between two plates, the bottom plate remains stationary and the top plate rotates at a certain frequency $\omega$, generating a time dependent shear strain $\gamma(t)=\gamma \sin (\omega t)$ on the sample, while the time dependent shear stress $\sigma(t)$ is quantified by measuring the torque that the sample generates on the bottom plate [175].

Figure 2.3 175] (b) shows the schematic representation of the time dependent stress response at a single frequency of an ideal elastic solid material, where the stress is proportional and always in phase with the applied sinusoidal strain deformation, hence the proportionality constant is equal to the shear modulus of the material.

On the other hand, in purely viscous fluids (Fig. $2.3 \mathrm{~b}$ ), the stress in the sample is proportional to the rate of strain deformation and the proportionality constant is the viscosity of the fluid. The applied strain and the measured stress are out of phase, with a phase angle $\varphi=\pi / 2$.

In viscoelastic materials (Fig. $2.3 \mathrm{~b}$ ), the response contains both, in-phase due to solidlike behaviours (red line) and out-of-phase liquid-like (blue dotted line) contributions. The purple line shows the total stress response and a phase shift $\varphi$ between that of solids and liquids, $0<\varphi<\pi / 2$. These contributions can quantify the viscoelastic behaviour by the storage modulus $G^{\prime}(\omega)$ (solid-like contributions of the stress response) and the loss

\footnotetext{
${ }^{1}$ Not to be mistaken with the bulk modulus.
} 
a)

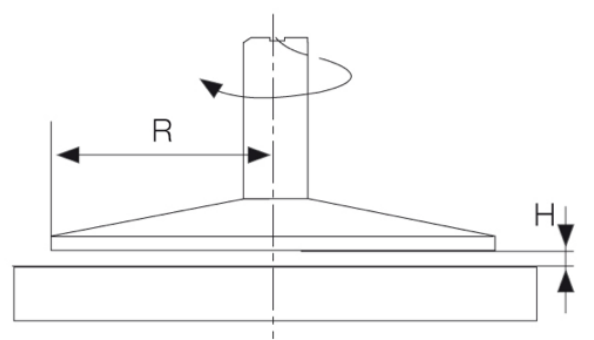

Elastic solid

b)

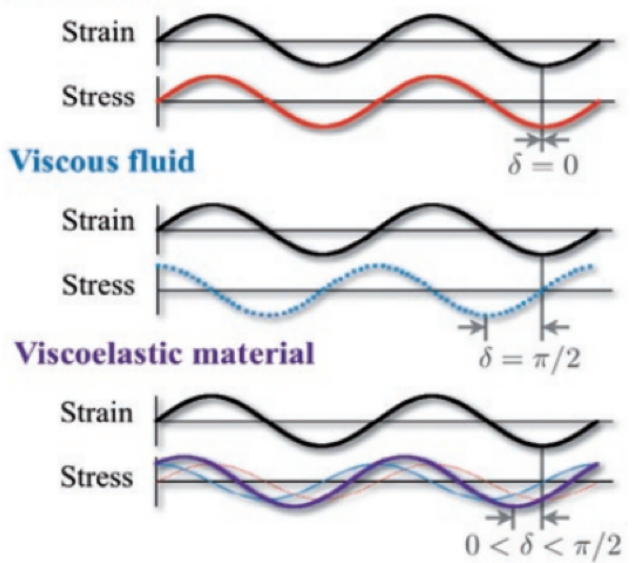

Figure 2.3: (a) Schematic representation of the shear rheometry set up. (b) Schematic representation of the stress response to oscillatory strain deformation for an elastic solid, a viscous fluid and a viscoelastic material. Taken from [175].

modulus, $G^{\prime \prime}(\omega)$ (fluid-like response).

When energy is introduced to the material because of a deformation, the viscous response absorbs this energy and by internal friction, it is dissipated in the form of heat. This is represented by the loss modulus $G^{\prime \prime}$. On the contrary, the elastic response stores this energy in the deformed material and gives the material the driving force for reforming the structure into its original shape. This is the storage modulus $G^{\prime}$.

For a sinusoidal strain deformation $\gamma(t)=\gamma_{0} \sin (\omega t)$, the stress response of a viscoelastic material is given by $\sigma(t)=G^{\prime}(\omega) \gamma_{0} \sin (\omega t)+G^{\prime \prime}(\omega) \gamma_{0} \cos (\omega t)$.

The complex shear modulus $G^{*}(\mathrm{~Pa})$ describes the viscoelastic behaviour of a material as $G^{*}=G^{\prime}+i G^{\prime \prime}$, where the real part is in phase with the strain and whose imaginary part is $90^{\circ}$ out of phase with it.

The storage and loss moduli can be related to the measured phase angle and the modulus amplitude $\sigma_{0} / \gamma_{0}$ at each frequency $\omega$.

$$
G^{\prime}=\frac{\sigma_{0}}{\gamma_{0}} \cos \varphi
$$




$$
G^{\prime \prime}=\frac{\sigma_{0}}{\gamma_{0}} \sin \varphi
$$

Rheological experiments measure $G^{\prime}$ and $G^{\prime \prime}$ as a function of omega since if the soft material is solid-like or liquid-like, the behaviour will depend on the time scale at which it is deformed.

We performed frequency sweeps from $0.1-1 \mathrm{~Hz}$ to describe time-dependent behaviours of the sample. The oscillation frequency was increased step-wise from one measuring point to the next while keeping the strain amplitude $\gamma_{A}$ constant at $1 \%$. 


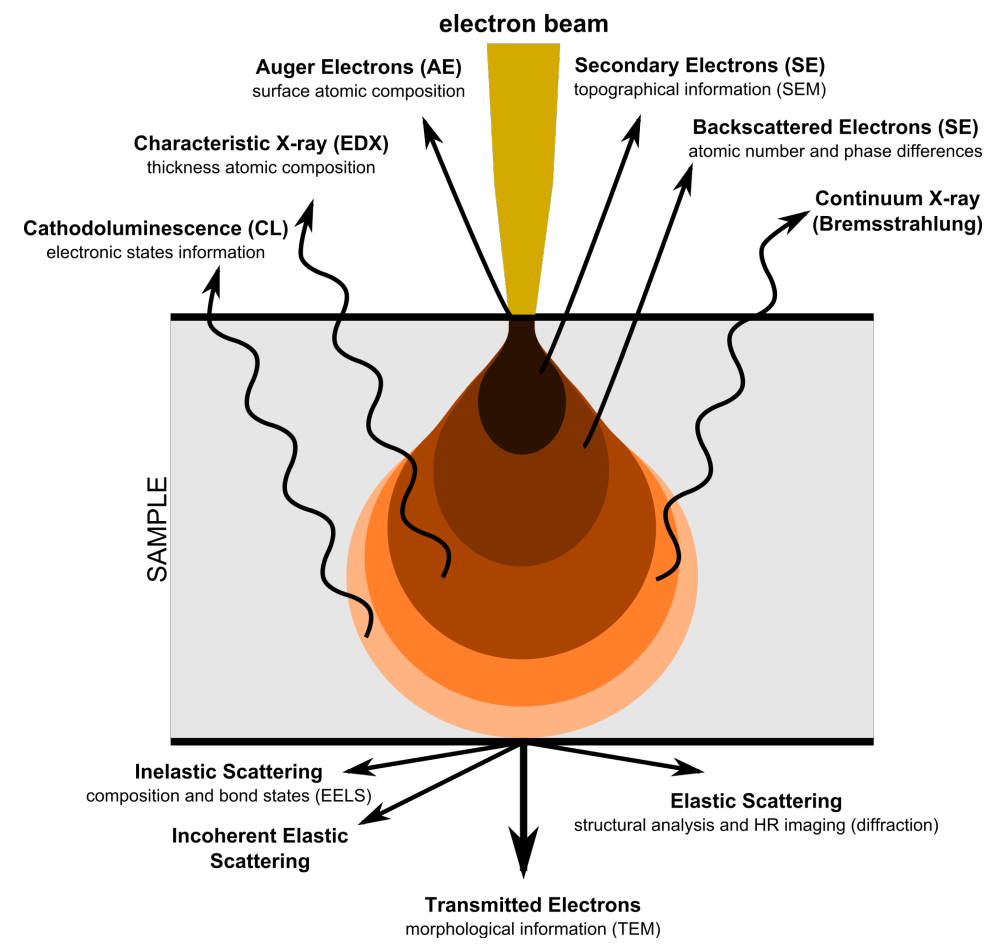

Figure 2.4: Schematic representation high energetic electrons interacting with the sample. Taken from 176.

\subsection{Electron microscopy}

Electron microscopy operates with the same basic principles of light microscopes, using electrons instead of light which enhances the resolution.

The accelerated electrons used in this microscopy carry significant amounts of kinetic energy that are decelerated into the solid sample, dissipating their energy in a variety of signals due to the electron-sample interactions as shown in figure 2.4 .

The signals that are used to image samples are secondary electrons and backscattered electrons (BSE). Secondary electrons show morphology and topography of the samples and BSE illustrate contrasts in composition in multiphase samples based on atomic number and related to relative density.

Other signals are diffracted backscattered electrons (EBSD used to determine crystallographic structures and orientations, visible light (cathodoluminescence, CL), heat and 
X-rays used for elemental analysis.

\subsubsection{EDX}

X-rays are produced by incident electrons that collide with electrons in the sample and return to lower energetic states called electron relaxation. Electrons in the s or p orbital are excited and become free electrons creating a relaxation cascade of electrons form higher orbitals into the free state of the low orbital. These transitions have a characteristic energy for different the elements. Because of this, SEM can show spatial variations in chemical compositions and acquire elemental maps. Elements from Be (atomic number 4, only in vacuum) to $\mathrm{U}$ (atomic number 92) can be detected.

\subsubsection{SEM}

SEM is a microscopy technique that uses a probe of accelerated electrons with energies up to $40 \mathrm{keV}$ focused on a specimen and scanned along a sample.

In 1926, the development of electron optics by studies of Busch on the trajectories of charged particles in axially symmetric electric and magnetic fields, lead to the fabrication of the first 'scanning microscope' in 1935 by Knoll. This microscope did not have a fine focused beam probe, which limited the resolution to $100 \mathrm{~mm}$ [177]. Nowadays, conventional SEM techniques have a magnification ranging from 20X to approximately $30,000 \mathrm{X}$ and a spatial resolution of 0.50 to $100 \mathrm{~nm}$.

Figure 2.5 (a) shows the structure of a SEM. Here, an electron beam is emitted thermionically from an electron gun that has a tungsten filament cathode. The electron beam is focused by condenser lenses to a spot of $0.4 \mathrm{~nm}$ to $5 \mathrm{~nm}$ in diameter. The beam passes through scanning coils or deflector plates to deflect the beam in the $\mathrm{x}$ and $\mathrm{y}$ axes and scan the sample. The beam current that is absorbed by the sample is detected to create 
a)

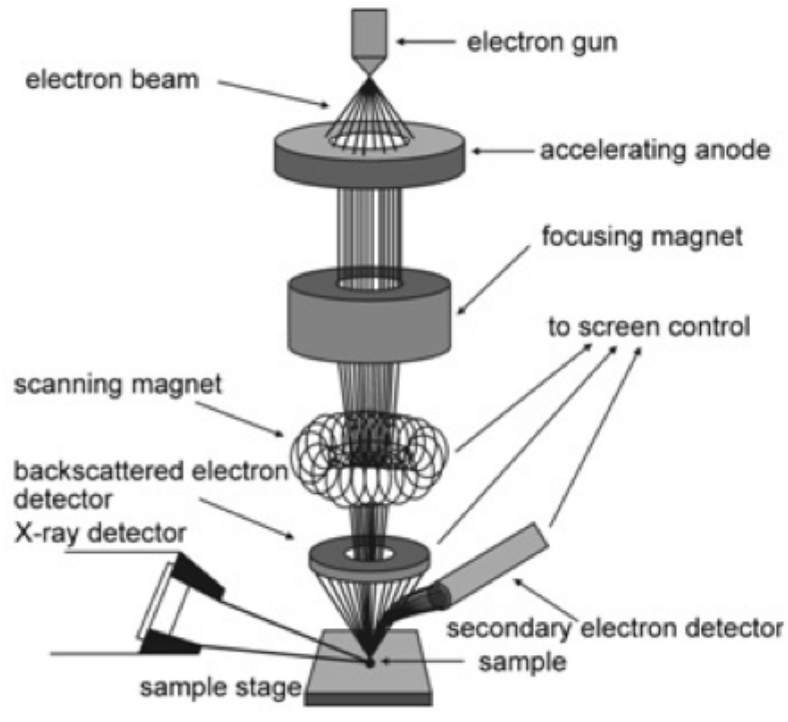

b)

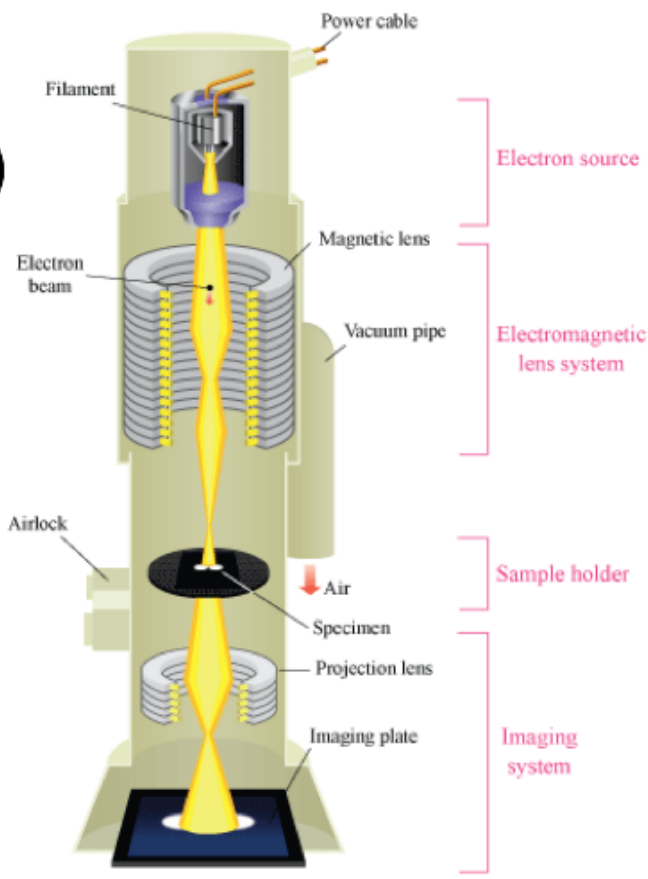

Figure 2.5: Schematic representation of (a) scanning electron microscope (taken from $[178$ ) and (b) transmission electron microscope (taken from 179 ).

images of current distribution. Amplifiers are used to enhance the signal and displayed as variations in brightness. These pixels are synchronized with the position of the beam, resulting in an image of the distribution of intensity emitted from the scanned area of the sample.

\subsubsection{TEM}

The first TEM microscope was developed by Ernst Ruska and Max Knolls in 1931.

In this microscope, the electron beam is emitted from an electron gun in the top of the microscope and travels through vacuum into electromagnetic lenses to focus the electrons into a coherent and thin beam. This beam then hits the sample, scattering the electrons in a representative way that depends on the sample thickness, density and its electron transparency, as shown in figure 2.4. The part of the beam that is transmitted is focused by an objective lens into an image on phosphor fluorescent screen or charge coupled device 
(CCD) camera as shown in figure 2.5 (b).

The main differences between these two microscopy techniques are that SEM uses coils to scan the sample using a beam of electrons and collecting the scattered electrons, providing information on the sample's surface and composition. On the other hand, TEM collects the transmitted electrons after passing through the sample, offering information on the inner structure (crystal structure and morphology). Moreover, the reached spatial resolution of SEM is $\approx 0.5 \mathrm{~nm}$, while with the recent TEMs with aberration-corrections, the spatial resolution reaches $50 \mathrm{pm}$. 


\section{Chapter 3}

\section{Mechanical properties of natural}

\section{hydrogels for Tissue Engineering:}

\section{Alginate and Chitosan}

The work presented in this chapter has been submitted to the 'Journal of the Mechanical Behavior of Biomedical Materials' for publication in co-joint first authorship with Chih-Yao Chui for the work on alginate microbeads.

Similarly, the work on chitosan hydrogel is being prepared for publication in co-joint first authorship with Alba Piacenti.

Natural polymeric materials are widely used in tissue engineering and regenerative medicine due to their similarities with the ECM, availability, chemical versatility, inherent cellular interactions and cellular or enzymatic-controlled degradability 180. These materials are used to reproduce the chemical and physical environment of healthy tissues for creating artificial organs (skin, bone, cartilage) or 3D cell cultures, for research including drug 
testing, and models of tissues and disease. As presented previously, a key characteristic in the design of these materials is its mechanical properties, which is the focus of this chapter. I will describe the assessment of the mechanical properties of two natural hydrogels used in tissue engineering: alginate and chitosan.

In the first part, I describe a combination of swelling studies and the calculation of the

local reduced elastic modulus $(\tilde{E})$ using AFM based microindentations on alginate beads in order to assess their stability in Dulbecco's Modified Eagle's Medium (DMEM) cell culture medium for a period of 2 weeks. Furthermore, we also describe an unexpected and hitherto unreported property of chitosan hydrogels: its mechanical properties can be modulated by weak magnetic fields. Using AFM-based microindentations and oscillatory shear rheometry we demonstrate that both macroscopic and micron scale mechanical responses of the material are altered in the presence of a magnetic field.

\subsection{Alginate stability in modular Tissue Engineering applications}

\subsubsection{Alginate}

Alginate is a hydrophilic and anionic natural polysaccharide derived from bacteria and brown seaweed. It consists of linear anionic binary copolymers of $\alpha$-D-mannuronic acid (M block) and $\beta$-L-guluronic acid ( $\mathrm{G}$ block) residues in varying proportions, arranged in MM and GG blocks scattered with MG blocks [67] as shown in figure 3.1[181].

Alginate is widely used as a hydrophilic biopolymeric material in biomedical research due to its capacity to be ionically crosslinked by divalent ions as $\mathrm{Ca}^{2+}$ to form a gel under mild conditions without external heating or using strong organic chemicals 68 70]. Being a hydrogel, alginate can absorb water up to thousand times its dry weight, which 

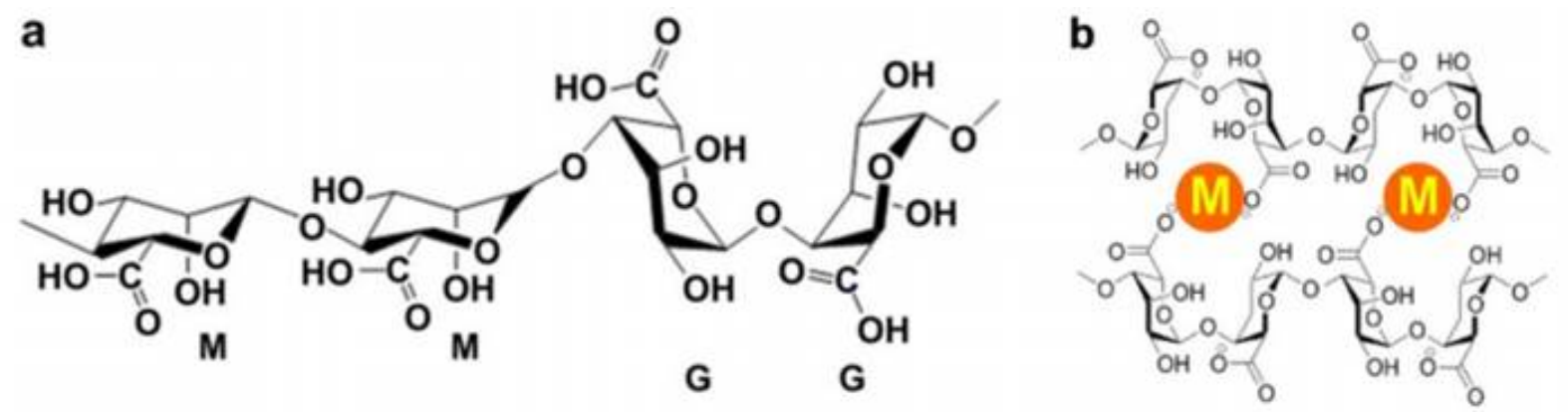

Figure 3.1: Linear anionic copolymers in alginate. (a) M blocks ( $\alpha$-D-mannuronic acid and G blocks ( $\beta$-L-guluronic acid) are arranged in MM and GG blocks. (b) MM and GG blocks are scattered with MG blocks. Image taken from 181.

reduces mechanical friction in vivo on surrounding tissue [65]. Alginate also possesses similar physiochemical properties to the native extracellular matrix [182 and acts as a semi permeable membrane allowing diffusion of nutrients and oxygen to cells while protecting them from mechanical stresses as well as from the host's immune system 183.

Because of this, and due to its thermal stability, alginate is widely used to make hydrogel beads in the presence of calcium cations via ionotropic gelation.

These beads have been designed as a bottom-up approach in modular TE for encapsulation of cells for allogenic cellular therapies [184, producing enzymes 185 and drugs [186] as well as in tissue engineering [187].

There are several concerns about the stability of the beads in in vitro studies due to non-gelling monovalent ions such as $\mathrm{Na}^{+}$present in cell culture environments [188, 189]. These ions exchange with $\mathrm{Ca}^{2+}$ within the bead, altering the mechanical integrity of the bead and leading to swelling, rupture and eventually, the dissolution of the beads and premature release of the cells. Furthermore, changes in bead properties could alter the final condition of cells as it has been shown that the material's mechanical properties have a strong influence on cell phenotype $52,54,190,191$.

To ensure long term culture, it is essential to understand stability, swelling behaviour and the mechanical properties of alginate beads under cell culture conditions. Previous 
reports have detailed these characteristics of alginate beads in buffer saline 189,192 194] or water [133, 195]. However, very few studies have measured these parameters in cell culture media 196. Despite similarities between these buffers, there are significant differences in the salt content between the buffer solutions and cell culture media, which could lead to different swelling behaviour or surface mechanical properties.

Bead mechanical stiffness has been previously investigated by measuring the bulk properties typically using a texture analyser [133, 195, 197 or micropipette aspiration [190]. Nevertheless, bulk properties do not reflect the properties of the material felt by cells at the micron scale. However, application of local nanoNewton $(\mathrm{nN})$ forces at the cellular level, can be achieved via indentation experiments using AFM. In addition, the measurements could be performed in a liquid environment mimicking a cell culture system.

AFM indentations have been used to characterise the mechanical properties of hydrogel scaffolds for TE [198]; alginate-based 3D scaffolds for tissue-engineered cartilage constructs [138], as well as investigating the correlation of matrix stiffness with breast cancer cellular activity 199]. AFM indentations have also been performed on alginate beads however, the indentations were not performed in cell culture conditions [200]. Moreover, most of these studies focus on the surface topography [201] or adhesion of the beads 200].

Relatively few studies investigated the mechanical properties of the microbeads [202]. Lekka et. al measured the Young's Modulus E (assuming a Poisson's ratio $\nu$ of 0.5 ) in $\mathrm{NaCl}$ buffer of crosslinked alginate beads of different formulations and coatings, for bead diameters up to $870 \mu \mathrm{m}$. They report $\mathrm{E}$ at the nanometer scale (tip radii of $50 \mathrm{~nm}$ ) with a high standard deviation resulting from both surface and batch heterogeneity. Yet this report consist of a single time point not assessing stability for a longer time period.

Furthermore, none of the studies mentioned above measured beads of a diameter suitable for cell encapsulation or microcarrier use. Alginate microbeads are typically around $200-400 \mu \mathrm{m}$ in diameter for cell encapsulation, in order to provide adequate diffusion of 
oxygen to the cells within the beads [187, 203, 204]. Similarly, microcarriers are typically between 90 - $300 \mu \mathrm{m}$ in diameter 205, 206 to maximise surface area to volume ratio while providing a sufficient surface area per bead to support cell growth [207, 208]. It has been shown that the dimensions of the sample would affect the stiffness values measured via AFM [209]. Guo et. al reported an increased E correlated with the decrease of the sphere diameter 209]. In the case of alginate beads, we observed a smaller diameter in the first experimental days, that correlates with a larger reduced Young's modulus $(\tilde{E})$. On following days, the stretching of the polymeric lattice due to hydration lead to a smaller $\tilde{E}$.

Hence, in order to quantify bead stability during cell encapsulation and microcarrier applications, it is vital to perform indentations in a cell culture environment with suitably sized beads.

In this chapter, AFM-indentations were used to quantify the variations in $\tilde{E}$ and how this relates to bead diameter of approx. $300 \mu \mathrm{m}$, suitable for both cell encapsulation as well as microcarrier applications. The $\tilde{E}$ measurement and swelling assessment in cell culture media were preformed over a period of 2 weeks which is the typical amount of time required in stem-cell culture 210212 for cell therapy.

Freeze drying is employed in the food and pharmaceutical industry for long term preservation and storage. The process involves removing water from a material through sublimation [213]. Successful freeze drying could extend shelf life and provide a pathway towards commercialisation for the alginate microbeads 123. It was previously shown that the porous gel networks of alginate beads could be preserved following freeze drying [214]. However, freeze dried beads are more fragile and susceptible to shear on their dry state 215]. Because of this, we used AFM indentation to identify changes in $\tilde{E}$ of rehydrated freeze dried alginate beads compared to freshly made counterparts, along with the effects of freeze drying in the bead re-swelling processes. 


\subsubsection{Materials and methods}

Alginate beads preparation by electrospraying, freeze drying and bead swelling studies were done by Chih-Yao Chui in a collaboration with the Biomedical Engineering department at the University of Oxford.

\subsubsection{Alginate beads Preparation}

Briefly, medium viscosity alginate (A2033 Sigma Aldrich) was dissolved to 1\% (w/v) in a $0.9 \%(\mathrm{w} / \mathrm{v}) \mathrm{NaCl}$ solution. Alginate beads were electrosprayed by passing the solution through a $30 \mathrm{G}$ blunt needle at $3 \mathrm{ml} / \mathrm{min}$ with an applied voltage of $7.5 \mathrm{kV}$ and electrode distance $2.5 \mathrm{~cm}$. Alginate microdroplets were gelled into beads in a $0.1 \mathrm{M} \mathrm{CaCl}_{2}$ bath for 1 hour.

Alginate beads were suspended in $2 \mathrm{ml}$ of low salt concentration (deionized) water at $13000 \mathrm{beads} / \mathrm{ml}$ in a 6 well plate. The beads were frozen down to $-40^{\circ} \mathrm{C}$ at $100 \mathrm{mtorr}$ before being freeze dried for $400 \mathrm{~min}$ at 200 mtorr. The temperature was ramped to $-20^{\circ} \mathrm{C}$ at 200 mtorr over 100 minutes. Finally, the temperature of the sample was ramped to $20^{\circ} \mathrm{C}$ over the next 100 minutes. Freeze drying was conducted using a BPS Genisis II freeze dryer (SP Scientific, USA).

\subsubsection{Assessment of Bead Swelling}

Alginate beads were added to low glucose (1g/l) DMEM with 10\% Foetal Bovine Serum and $0.1 \%$ Penicillin-Streptomycin. The beads were left in DMEM of 24 hours a period referred to as the conditioning period. Following this, the images of beads were captured using a Nikon TiE 2000 (Japan) fluorescence microscope. The bead diameters of 30 beads were measured each day using ImageJ (National Institutes of Health, USA), with the first measurement known as day 1 for a total of 14 days. Culture media was changed every 3rd day to mimic cell culture protocol. 


\subsubsection{AFM microindentation experiments}

\section{Bead gluing}

In order to consider an average $\tilde{E}$ of the alginate beads in DMEM, a $(20.0 \pm 0.3) \mu \mathrm{m}$ polystyrene bead (Sigma-Aldrich, 74491) (Young's modulus $E=3 G P a$ ) was glued to a Scanasyst-fluid+ cantilever (nominal spring constant $\kappa=0.7-1.4 \mathrm{~N} \mathrm{~m}^{-1}$, resonance frequency $f=150 \mathrm{kHz}$, Bruker) using sealant (Weicon) by indenting the glue and immediately sitting on top of a bead and lowering the stage to press the cantilever down until an apparent distortion of the cantilever was observed. The system was left in this position for 5 minutes and beads were allowed to dry.

Force vs. distance curves were obtained with a commercial MFP-3D AFM (Asylum Research, Santa Barbara, CA). For all the microindentation experiments, the cantilever was calibrated by indenting a glass surface at $1 \mu \mathrm{ms}^{-1}$ to obtain the amplitude inverse optical lever sensitivity (Invols). If we press against an infinitely stiff surface $\left(E_{\text {glass }}>>\right.$ $\left.E_{\text {cantilever }}, E_{\text {PEbead }}\right)$, the change in z piezo $(\Delta z)$ as a result of the cantilever bending $(\mathrm{d})$ depends linearly on how sensitive the cantilever is (C) by Hooks law $\Delta z=C d$, since there is no contribution from a deformation of an indented surface. This way, we can calculate the number of nm that the cantilever bent per number of volts of signal obtained $C=\frac{\Delta z}{d}=\frac{n m}{V}$. Once we have this value, we back calculated the spring constant of the cantilever using the thermal fluctuations method 216].

In subsequent experimental days, the laser sum was set in the closest value possible by modifying the alignment of the laser in order to have a similar position and amplitude Invols for similar experimental environments. The used cantilever was thoroughly rinsed with ultrapure water and submerged in ethanol for 10 minutes after each experiment, yet it is possible to encounter small discrepancies in cantilever stiffness in subsequent experiments, i.e. due to aggregation of residues at the cantilever surface. 
The cantilever was positioned on the highest part of an alginate bead by observing the piezo extension/retraction whilst moving the stage in both $\mathrm{x}$ and $\mathrm{y}$ directions. Fast indentations of the beads were performed $\left(45 \mathrm{\mu ms}^{-1}\right)$ to minimise the time-dependent behaviour of the beads due to viscoelasticity or poroelasticity. A force trigger point of $70 \mathrm{nN}$ was used to indent 30 beads, 50 times each. Indentations were conducted on day 1 , $5,8,12$ and 14 .

Preliminary experiments were conducted to optimise the procedure. Several challenges were encountered especially due to the propensity of beads to slide away from the cantilever during the 50 indentations. Several approaches were attempted to solve this problem. An initial trial was performed with the beads placed in a glass slide without a liquid cell but with a plastic 'pool' to contain the buffer. On a second trial, clear resin holders with holes of different diameters were designed using Autodesk inventor and 3D printed (Formlabs Form 2 ) in order to immobilise the beads during indentation. This procedure was complicated since the printing resolution rendered an excessive layer of polymer at the bottom that blocked light from the optical microscope coupled to the AFM, making it hard to identify the beads.

The solution to this problem was actually much simpler; submerging a glass slide in concentrated ethanol for 10 minutes and gently polishing the surface with tissue was enough to stop the beads from sliding uncontrollably, but did not fix the problem completely. Future experiments and roughness assessment via AFM imaging could potentially be done using of polylysine coated glass slides.

Another challenge encountered was to ensure minimal bead movement during the indentations in order to avoid significant changes in the contact angle and indentation area between the 2 spheres. The initial position of the piezo was observed prior indentation. In the case where bead movement was observed, the 50 indentations were reinitiated to ensure reproducibility. Furthermore, beads that presented a significant change of $\tilde{E}$ (as 
described later in 3.1.3.2, 3.3, were not considered. Because of this, the total amount of beads indented per experimental day varied from 35 to 45 to ensure a mean number of 30 beads taken into account in the statistics.

For all of the AFM indentation experiments described in this thesis, the fitting procedure to calculate the reduced elastic modulus $\tilde{E}$ was performed using the Hertz model for a sphere indenting a plane (eq. 2.1. depicted in figure 2.2. All experimental values are given in terms of $\tilde{E}$ since the Poisson's ratio of the polymeric materials used throughout this thesis are not unknown.

All the data analysis performed in this thesis was made using a custom-build analysis code implemented in Python. The contact point determination method was implemented by Dr. Jacob Seifert in Dr. Contera's lab at the University of Oxford. The fitting region varied depending on the sample. In this case, it was chosen from 10 to $400 \mathrm{~nm}$ which corresponds to the low forces region of the force curve and to small indentations in order to avoid non-linear elasticity effects [217].

For alginate beads indentations, a two way ANOVA $(p<0.05$, GraphPad Prism software) was used to determine significance of $\tilde{E}$ and bead diameter by comparing the mean differences between freshly made beads and freeze dried beads as independent variables during the two week measurement period. A post-hoc Tukey test was used to identify the significance. Bootstrapping was performed on the stiffness values in order to estimate the standard error of the mean. Each bootstrap contained 30 samples from the data with replacement, a total of 100 bootstraps were performed.

Bootstrapping is a resampling technique used to estimate statistics on a population by sampling a data set with replacement. It consists on making a new sample from 100 random picks from the data set. The standard error of the mean (how far the sample mean is from the population mean) is obtained from this data set. This approach was chosen since there are many potential errors in the calculated $\tilde{E}$ such as bead indentation area 
and fabrication variation of beads and it is important to assess variation within the whole population instead of each data given by the standard deviation.

\subsubsection{Results}

\subsubsection{Bead Swelling}

The spherical alginate beads created via electrospraying are shown in figure 3.2 (a) in an optical microscopy image, with a diameter of $(217 \pm 20) \mu \mathrm{m}$ on average. The beads swelled to an average of $(310 \pm 20) \mu \mathrm{m}$ after the addition of DMEM (figure 3.2 (b). After freeze drying, the beads shrunk into small spheres which appeared to clump together with an average diameter of $(90 \pm 10) \mu \mathrm{m}$ although the beads re-swelled into distinct spherical beads of diameter of $(267 \pm 10) \mu \mathrm{m}$ following addition of DMEM (figure 3.2 (c). Freshly made beads displayed significant swelling of the bead from day 1 to day 2 (figure $3.2 \mathrm{~d}$ ). However, the bead size remained stable over the rest of the measurement period. This initial swelling was also observed in the freeze dried beads, however the diameter on day 2 was still significantly smaller compared to the diameter of day 8, 10, 11 and 14 (figure 3.2 d). After day 2, the bead size remained stable. Finally, bead diameter of the re-swelled freeze dried beads was significantly smaller compared to the freshly made beads on all of the days.

\subsubsection{2 $\tilde{E}$ of alginate beads}

$\tilde{E}$ is related to the Young's modulus by equation 2.2 and describes tensile elasticity of a material. All AFM indentation results in this thesis are reported in terms of $\tilde{E}$ since the $\nu$ is Poisson's ratio of the materials are not known.

The $\tilde{E}$ of the beads was calculated from indentation experiments as described previously (section 3.1.2.3 . Figure 3.3 shows the results of the 50 indentations on two freshly made 

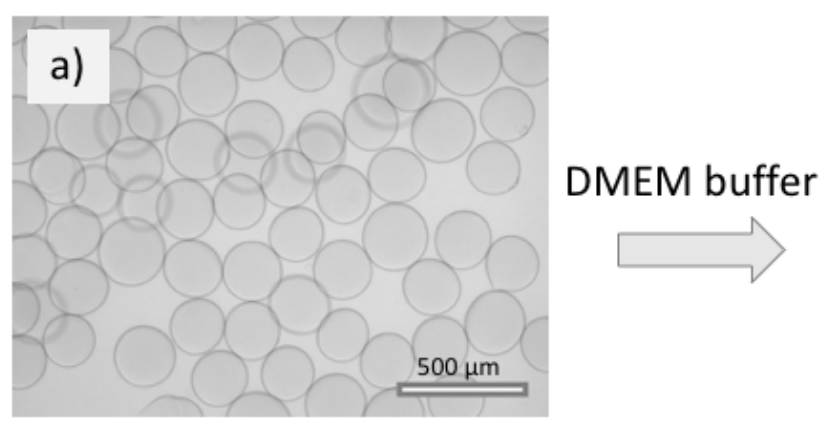

d)

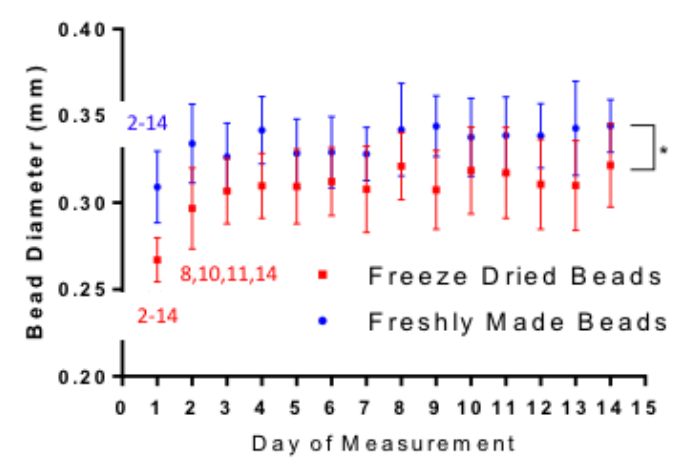

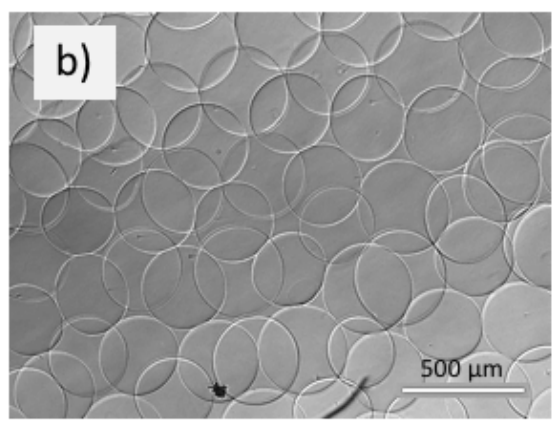

Freeze drying
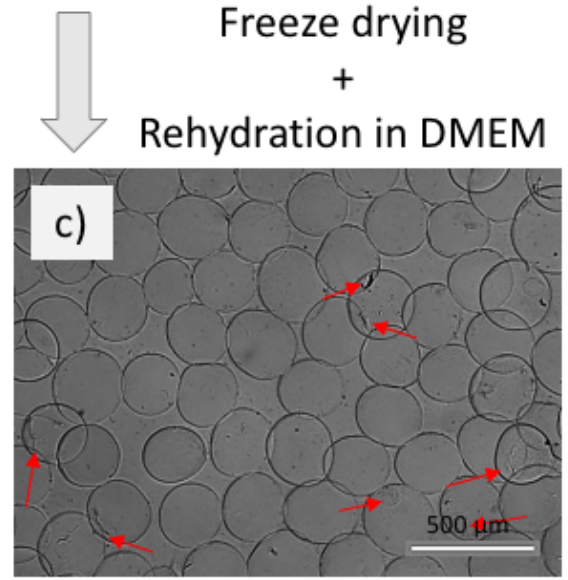

Figure 3.2: Changes in bead size and morphology under different conditions observed with optical microscopy. (a) Alginate beads in $\mathrm{CaCl}$ solution created by electrospraying, (b) Swelling of alginate beads upon immersing in DMEM media for 24 hours, (c) Re-swelling of the freeze dried beads after the 24 hour conditioning period. Rough surfaces were observed on the beads (indicated by red arrows). (d) Bead diameter in DMEM culture media over a 14 day period for freshly made beads (blue) and freeze dried beads (red). The days in which the bead diameter is significant are shown by numbers above the measurement. ${ }^{*}$ denotes the freshly made beads were significantly larger compared to the freeze dried beads. Error bars show standard deviation. Images and graph were made by Chih-Yao Chui. 

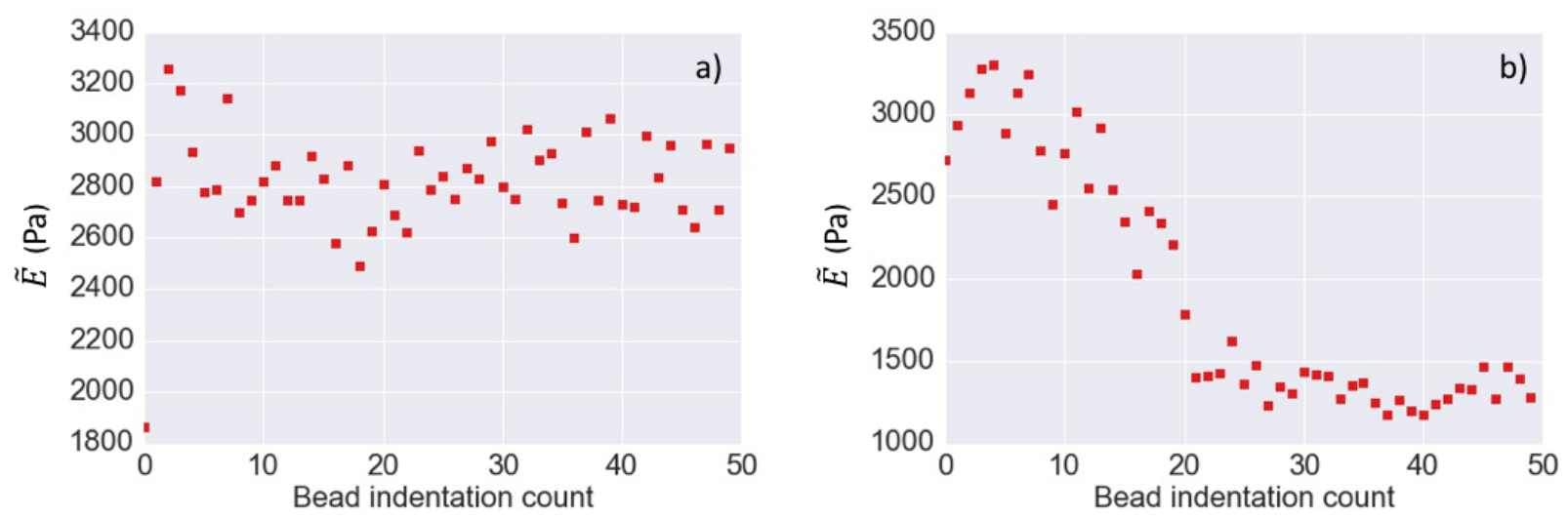

Figure 3.3: Obtained $\tilde{E}$ value of 2 different freshly made beads indented 50 times each. (a) Non significantly different dispersion of $\tilde{E}$ values, ensuring minimal bead movement. (b) Significantly different dispersion of $\tilde{E}$ due to bead sliding.

beads. (a) the data show a non significantly different dispersion of $\tilde{E}$ values obtained throughout 50 indentations, ensuring minimal bead movement. This kind of behaviour is representative of all the results shown in this chapter (summarised below in figure 3.4). Figure 3.3 (b) shows a significantly different dispersion of $\tilde{E}$ after the indentation number 20, due to a change of position of the alginate bead because of sliding and consequent change in contact angle and contact area. This kind of behaviour was discarded from the analysis.

Figure 3.4 (a) shows $\tilde{E}$ values of the freshly made beads was significantly higher on day 1 compared to the rest of the time points. However, there was no significant change in $\tilde{E}$ over the rest of the 2 -week period. The freeze drying process resulted in a significantly higher $\tilde{E}$ compared to freshly made beads, until the last couple of experimental days. Furthermore, the $\tilde{E}$ of the freeze dried beads decreased significantly from day 8 to 12 while remaining non-significant from day 1 to 8 (figure 3.4 (b)). 

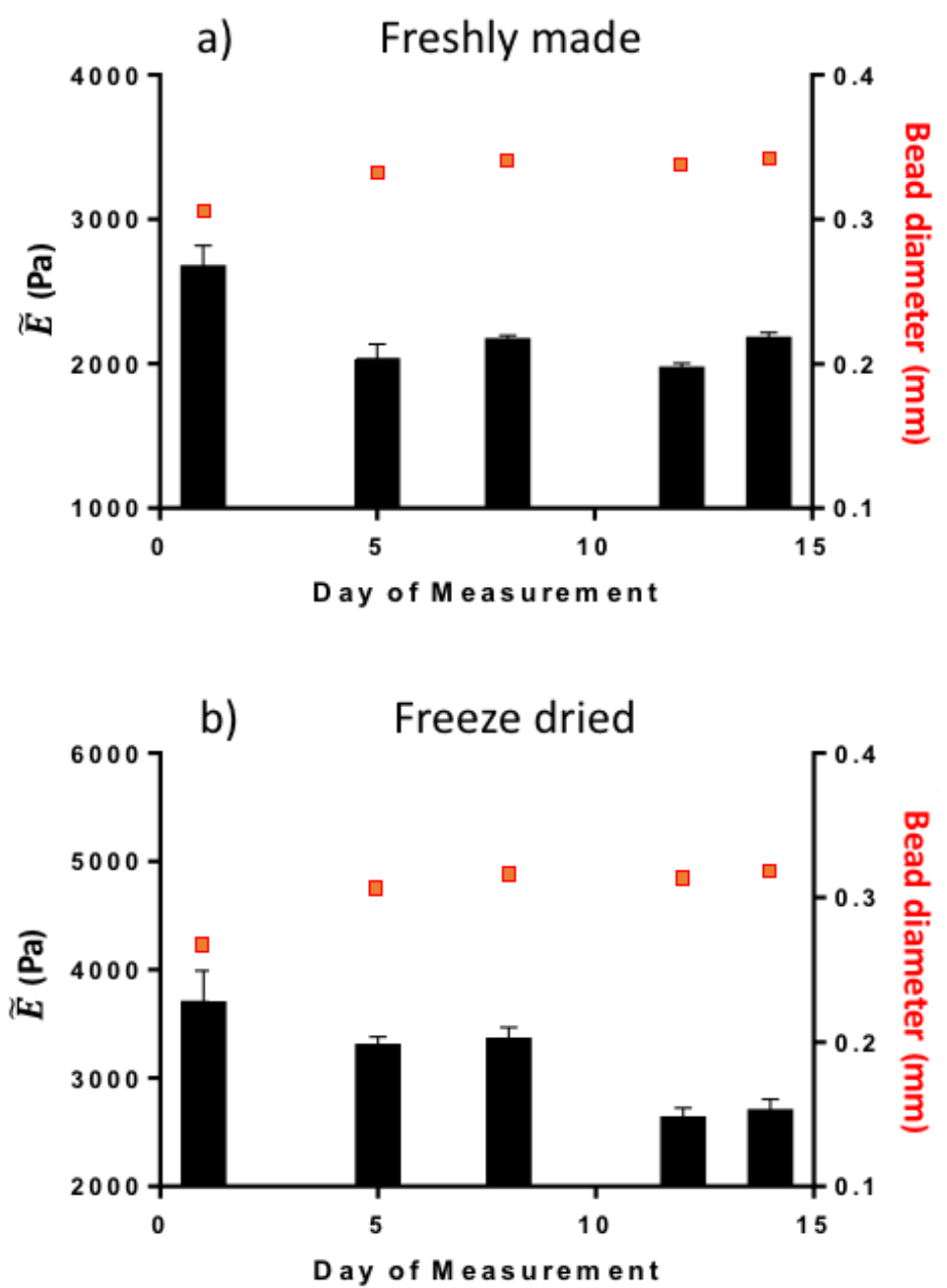

Figure 3.4: Reduced modulus measured on day 1, 5, 8, 10 and 14 post conditioning, of freshly made beads (a) and freeze dried beads (b). Error bars show standard error to the mean. Bead diameter from bead swelling measurements were plotted on the secondary axis. 


\subsubsection{Discussion}

We measured the $\tilde{E}$ of alginate beads using AFM-indentation experiments in order to assess the mechanical stability of these during a period of two weeks. The 24 hour conditioning period allowed the beads to equilibrate in the medium. Moreover, this equilibration period also allowed proteins in the medium to form a coat on the surface of the carriers to enable cell attachment 218. Furthermore, in the case of the freeze dried alginate beads, this time allows beads to re-swell in the media.

\subsubsection{Bead Swelling}

Divalent cations such as $\mathrm{Ca}^{2+}$ interact with both $\mathrm{M}$ and $\mathrm{G}$ blocks of alginate. With $\mathrm{G}$ blocks, $\mathrm{Ca}^{2+}$ packs in the interstices between coordinated polymer chains creating a $3 \mathrm{D}$ network. This model is known as the 'Egg-box' model and its formation causes the alginate to gel 219]. However, alginate beads are susceptible to non-gelling monovalent ions such as $\mathrm{Na}^{+} 192$ present in cell culture media. These ions make alginate beads swell and eventually dissolve 220, 221]. $\mathrm{Na}^{+}$initially exchanges with $\mathrm{Ca}^{2+}$, binding to $\mathrm{M}$ groups and causing an electrostatic repulsion between $\mathrm{COO}^{-}$groups which relaxes the chain. This allows the surrounding medium to penetrate into the bead, causing swelling and eventually $\mathrm{Ca}^{2+}$ in G groups are exchanged and the Egg box structure disintegrates, breaking the bead [192].

In this study, $\mathrm{Ca}^{2+}$ exchange with $\mathrm{Na}^{+}$from the DMEM buffer reached equilibrium on day 2 for the freshly made beads since no further significant swelling was observed after that. A similar swelling behaviour in saline incubation has been reported previously 189 with rapid swelling for the first two days of incubation, followed by a constant diameter for over the 14 day period. This was not the case in PBS, where the $\%$ weight change increases rapidly due to water uptake up to 60 minutes of incubation. The weight of the beads remain stable for several hours, unlike the diameter, the bead weight began to decline at around 200 minutes post incubation indicating degradation of the bead [194]. 
This is due to the fact that unlike saline, PBS also contains phosphate ions which destabilises $\mathrm{Ca}^{2+}$ linkages 222. Although DMEM also contains phosphates, in this study the beads remained intact by the end of the two weeks; unlike PBS, DMEM contains $\mathrm{Ca}^{2+}$ ions (Sigma-Aldrich, n.d.) which can be expected to counterbalance the ion exchange to a certain degree.

Freeze dried beads, in contrast, appeared to equilibrate later since the diameter on day 2 was still significantly smaller compared to the diameter on days 8, 10, 11 and 14, possibly due to the incomplete re-swelling following the freeze-drying process.

In this study, the freeze dried-beads failed to reach the original diameter during the entire 14 day culture period. This could be due to longer equilibration of ion exchange because of a higher polymeric density and crosslinking ratio as a consequence of the higher porosity introduced in the beads.

\subsubsection{Mechanical Properties measurement by AFM microindentation.}

Bead swelling has been related to bead stability, yet, changes in $\tilde{E}$ could also be an indicator of bead stability since the mechanical properties of hydrogels normally alter prior to any physical change [190]. Because of this, the performance of both tests was done in parallel.

\subsubsection{Hertz model}

The compressed materials are required to be linearly elastic and follow Hooke's law for Hertz's theory to be valid 223. Because of this, the chosen fitting region of the force vs distance curves was chosen to be 10 to $400 \mathrm{~nm}$, since it corresponds to low forces and small indentations in order to avoid non-linear elasticity effects 217] and fall within the Hertzian limit.

Alginate beads behave viscoelastic and poroelastically as the water in the beads is pushed out during deformation in a time-dependent manner. However, it has been shown 
that at high compression speeds, the time-dependent behaviour could be neglected [133].

Furthermore, the standard deviation of the $\tilde{E}$ values of the 50 indentations of each bead was low, with a relative standard deviation of approx. 10\%, suggesting that the results were time-independent, hence any viscoelastic responses could be neglected as they will be

minimal compared to $\tilde{E}$. Additionally, small a dispersion in $\tilde{E}$ values can be an indicative of the lack of bead movement in between each indentation, indicating experimental reproducibility (see figure 3.3).

\subsubsection{Indentation experiments}

In the case of the freshly made beads, $\tilde{E}$ was significantly higher on day 1 , while remaining the same for the rest of the two weeks. This is likely due to the bead size being significantly smaller on day 1. The swelling indicates weakening of the $\mathrm{Ca}^{2+}$ gel network, hence the $\tilde{E}$ of the beads would decrease. However, as the ionic exchange reaches equilibrium, the $\tilde{E}$ would stabilise similarly to the diameter.

On the other hand, the $\tilde{E}$ of the freeze dried beads was significantly higher compared to freshly made beads until the last two experimental days, where it decreased to a value similar to the freshly made beads. Freeze drying will create porosity by sublimation of ice crystals embedded within the polymeric lattice, hence the hydrogel polymer will have a greater density, a more crosslinked lattice and rougher surface. It is likely that the higher crosslinking took longer to reach equilibrium, hence softening (lower $\tilde{E}$ ) similar to the initial $\tilde{E}$ value of freshly madre beads was observed in the last experimental days.

it is important to note that due to the creation of porosity and surface roughness as a result of freeze drying, the standard deviation of the measured $\tilde{E}$ increased in comparison to freshly made beads. This is as expected since porosity introduces free space inhomogeneities.

These results are comparable to previously reported E measurements of alginate beads 
by Lekka et. al 202 discussed in the introduction. The reported assessed beads closest to our formulation (1.7\% sodium alginate Keltone HV, $425 \pm 15 \mu \mathrm{m}$ bead diameter, calcium cation) has an $\mathrm{E}$ of $0.2-1.4 \mathrm{kPa}$, within the same order of magnitude to our report (considering $\nu=0.5$ ) despite several differences in the experimental technique such as the indentation area.

\subsubsection{Conclusions}

In this chapter, we described the fabrication, swelling behaviour and mechanical characterisation of calcium alginate beads suitable for cell encapsulation and microcarrier purposes. In order to ensure long term culture in cell culture conditions, the stability of the beads has to be assessed.

This was achieved through successful measurement of the local mechanical properties of freeze dried and freshly made alginate beads via AFM microindentations in parallel to measuring the bead diameter by optical microscopy over the course of two weeks. There is a correlation between bead size (volume) and measured $\tilde{E}$ modulus over time that is also observed on the freeze dried beads, where initial smaller diameter accounts for higher $\tilde{E}$.

Furhtermore, freshly made beads had a significantly higher $\tilde{E}$ on the first day, along with the bead size being significantly smaller. This can be explained because of ion exchange of $\mathrm{Ca}^{2+}$ ions that crosslink the alginate network with monovalent ions such as $\mathrm{Na}^{+}$from DMEM. This weakens the alginate $\mathrm{Ca}^{2+}$ gel network allowing more water into the bead, causing swelling and lowering $\tilde{E}$. Equilibrium of this ion exchange was reached when swelling did not change significantly from day 2 and was confirmed and consistent with $\tilde{E}$ varying non-significantly over the remaining time points.

We have also measured the diameter and $\tilde{E}$ of electrosprayed beads that underwent a freeze-drying cycle. The results of the study showed that re-swelled freeze dried beads have a higher $\tilde{E}$ modulus and smaller diameter, in comparison to freshly made beads in 
the first 10 days of the experiment.

This difference could be due to the formation of porosity during freeze drying, which also explains the rougher surface of the beads, smaller diameter and higher average $\tilde{E}$ moduli. Higher porosity would increase the density and crosslinking of the material, taking longer for the ion exchange to equilibrate, this would lead to a significant decrease of $\tilde{E}$ on the last couple of experimental days (from day 10) to a value much closer to the initial freshly made beads moduli. Future experiments, should involve the quantification of porosity change due to the freeze drying process.

The elasticity coupled with diameter changes were conducted in a mimicked cell culture environment and time frame. Despite the need of a coating layer for cell attachment in cell culture applications, our results provide information on the overall stability of alginatebased beads for biomedical applications such as microcarrier cell culture and cell encapsulation. Our results pointing towards the capacity of using freeze drying without affecting the mechanical stability of the beads. 


\subsection{Chitosan hydrogel mechano-magnetic coupling: An unexpected property}

\subsubsection{Introduction}

Polymeric materials whose mechanical properties can be controlled by external magnetic fields are attractive in a wide range of applications in e.g. artificial muscles [109], sensors [110], micro-robots 112, 224 and micropumps [113, 114], controllable membranes [111, 116], adaptive tuned vibration absorbers, stiffness tunable mounts and automobile suspensions 225]. In biomedicine magnetically tuneable mechanical properties of polymeric materials find applications in several areas including biosensing, drug delivery and regenerative medicine [108]. In tissue engineering, magnetic control of properties of the biomaterials used to seed the cells in 3D cultures, might be used to improve the diffusion of nutrients, drugs and growth factors and facilitate the growth of healthy tissue by controlling cellular responses 226].

The use of magnetic fields in biomedical applications is particularly advantageous due to its not invasive nature, it can be spatially and temporally controlled, and it is easy to implement in aqueous environments and does not affect biological activity 227].

The technological route to add magneto-mechanical functionality to polymeric materials has been the incorporation of micron and nano-sized magnetisable particles embedded in the polymer to form composites such as magneto-sensitive elastomers 225] and magnetic composites for tissue engineering 226].

To the best of our knowledge, there have not been reports of non-composite polymeric materials whose mechanical properties can be directly modulated by magnetic fields. In this section of the chapter, we describe that the mechanical properties of the extensively used chitosan hydrogel can be modulated by weak magnetic fields without further modification 


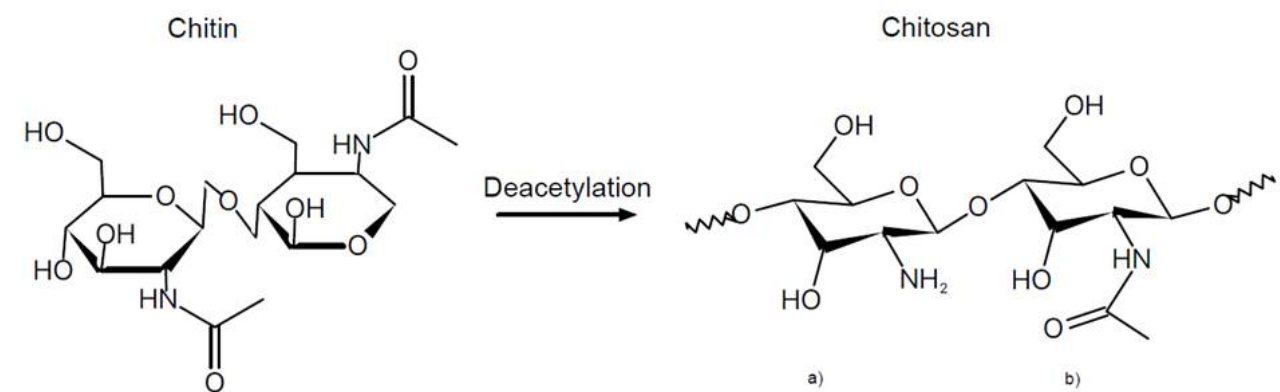

Figure 3.5: Deacetylation reaction to obtain chitosan from chitin. a) 2-amino-2deoxy- $\beta$-D-glucopyranose, b) 2 -acetamide-2-deoxy- $\beta$-D-glucopyranose

of the material.

\subsubsection{Chitosan}

Chitosan is a one of the most abundantly available biopolymers and is primarily obtained from the deacetylation of chitin present in the shells of shrimps as shown in figure 3.5. The main drawback of chitin is the presence of hydrophobic acetyl groups that make it hardly soluble. When these groups are removed, the free amine groups in the chitosan molecules make them soluble in acidic $\mathrm{pH}$ solutions $(\mathrm{pH}<6.5)$ and give chitosan its cationic behaviour, compared to many other neutral or negatively charged polymers [228]. The cationic amino groups react with multi-valent anions to form hydrogels.

Chitosan is inexpensive, biodegradable, biocompatible, hypoallergenic, antibacterial, and bioadhesive. It is widely used in the treatment of wastewater [229], in cosmetics and cosmeceuticals 230], food preservation and packaging [231] to promote plant productivity and prevent the attack of pathogens in crops 232. It is used in wound treatment 233] since it is known to promote the healing process of soft and hard connective tissues [234], in drug delivery 235, 236] and it is one of the most successful materials in tissue engineering 237, 238. In physiological environments various enzymes, such as chitosanase and lysozyme, degrade chitosan and form harmless products [239]. The global chitosan market is expected 
to reach USD 17.84 billion by 2025 .

\subsubsection{Chitosan Crosslinking}

Due to the polycationic nature of chitosan in acidic conditions, hydrogels can easily be fabricated because of the presence of electrostatic interactions. There are three main methods to crosslink chitosan hydrogels: physical gelation, coordination with metal ions, and chemical crosslinking [240].

Physical gelation is based on non-covalent reversible interactions that occur between polymer chains, such as electrostatic (with negatively charged molecules, such as phosphates, sulfates and citrates ions) and hydrophobic interactions or hydrogen bonds. Coordinate-covalent bonds can also be achieved via metal ions as Pt (II), Pd (II), and Mo (VI). Lastly, covalent bonding between polymer chains can also be achieved, rendering much more stable hydrogels.

In this thesis I describe the fabrication of chitosan hydrogels using Genepin as a crosslinking agent. Genipin is a natural chemical extracted from the fruit of Gardenia jasminoides used in traditional Chinese medicine to treat diabetes miellitus type 2 [241]. It is commonly used as a crosslinker of chitosan due to its low toxicity, hence preferred over other toxic commonly used agents such as glutaraldehyde [242] and many other synthetic crosslinking reagents.

Figure 3.6 shows the reaction of the amine groups of chitosan with the genipin structure interconnected with the highly stable intermolecular cross-covalent bonds linking the chitosan chains 243. 


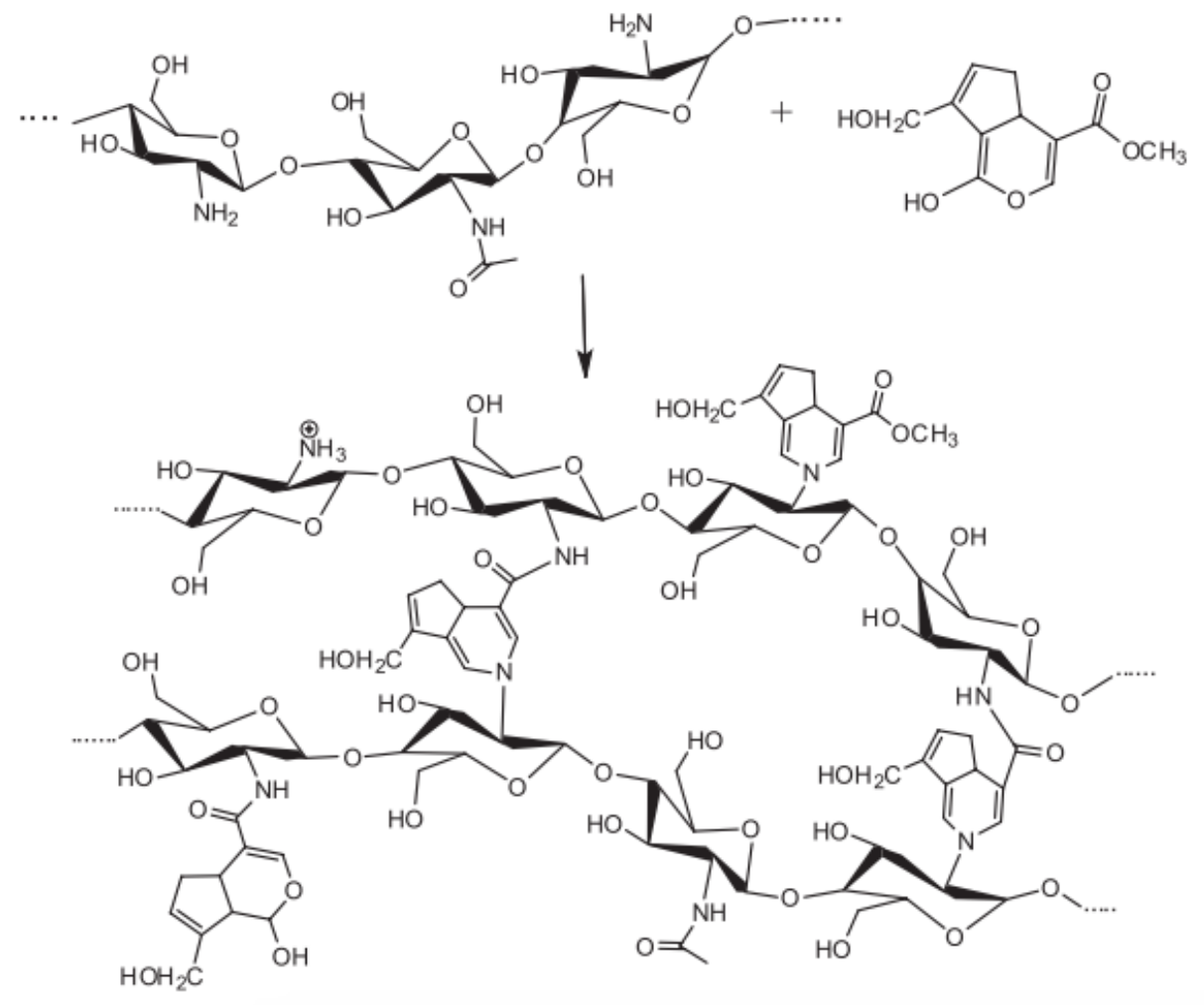

Figure 3.6: Crosslinking reaction of amine groups in chitosan with genipin. Taken from 243 


\subsubsection{Materials and methods}

\subsubsection{Chitosan scaffolds fabrication}

Chitosan hydrogels were prepared by freeze drying. $0.5 \% \mathrm{w} / \mathrm{v}$ of poly(D-glucosamine) deacetylated chitin of medium molecular weight (157.5 kDa) (Sigma Aldrich) was dissolved in $0.05 \mathrm{M}$ acetic acid by magnetic stirring for 6 hours. The $\mathrm{pH}$ was adjusted to 4 by adding $1 \mathrm{M} \mathrm{NaOH}$ and undissolved chitosan was removed by centrifugation for $5 \mathrm{~min}$ at 13,000 rpm.

Genipin (Challenge Bioproducts) was used as crosslinker and added to the chitosan dilution at $0.23 \mathrm{M}$. The solution was magnetically stirred for 1 hour to ensure homogeneity.

All dispersions were frozen in $1.3 \mathrm{~cm}$ diameter molds at $-80^{\circ} \mathrm{C}$ for 30 minutes and dried using a lyophiliser (VirTis Bench Top $2 \mathrm{~K}$, SP Industriesat $-75^{\circ} \mathrm{C}$ and an interval of 10 to 0.1 mtorr for 30 hours.

The resulting $1.3 \mathrm{~cm}$ in diameter by $0.5 \mathrm{~cm}$ in thickness sponges were then crosslinked at $37^{\circ} \mathrm{C}$ for $24 \mathrm{hrs}$. After this, they were fixed to a glass slide by adding a two part double bubble epoxy (Loctite) to a double sided tape stuck to the glass surface. The sponge was flipped upside down, placed in the epoxy layer and dried overnight. The sponges were submerged in ultrapure water and allowed to equilibrate for a week before performing any experiments. Indenting the material before this time resulted more experimentally challenging. It was harder to obtain full force curves besides the cantilever getting stuck in the polymeric mesh more often.

Polydimethylsiloxane (PDMS, Sylgard 184 Dow-Corning) controls were fabricated using a mixing ratio of 10:1 for elastomer and curing agent. After homogenising, the solution was poured in aluminium foil molds and desiccated for 30 minutes; then they were cured at $110^{\circ} \mathrm{C}$ for 6 hours.

All solutions were stored in a fridge at $5^{\circ} \mathrm{C}$ and hydrogels at RT. 


\subsubsection{Local mechanical characterisation: AFM-microindentations}

AFM- based microindentations were performed to measure the mechanical properties of the hydrogels. A $49.72 \mu \mathrm{m}$ glass bead (Whitehouse Scientific) was glued to a AC240TS-R3 cantilever (Olympus, nominal $\kappa=2 \mathrm{~N} \mathrm{~m}^{-1}$ ) as described previously (section 3.1.2.3). The cantilever was calibrated using the thermal oscillation method [216, 244].

Force vs. distance curves were obtained in 1 x Posphate buffered saline (PBS) (Sigma Aldrich; $0.138 \mathrm{M} \mathrm{NaCl}, 2.7 \mathrm{mM} \mathrm{KCl}, \mathrm{pH} 7.4$ ). Initial experiments were performed in the presence of a $85 \mathrm{mT}$ static $\vec{B}$ from a permanent NdFeB magnet that was removed and placed manually. This resulted challenging due to the lack of control of the magnet and possibility of slightly moving the indented position. This problem was solved by using an electromagnet (First4magnets) fitted underneath the sample $(30 \mathrm{~V}, 0.38$ amps, B $=45 \mathrm{mT}$ at the sample height) by lifting the AFM head with costume made metallic legs of $10 \mathrm{~cm}$ in height. All data shown in this thesis was obtained using this electromagnet.

Fast indentations were performed $\left(50 \mathrm{\mu m} \mathrm{s}^{-1}\right)$ to minimise the time-dependent behaviour. A force trigger point of $100 \mathrm{nN}$ was used. 10 positions around the polymer were tested; for each position, a total of 100 force curves were recorded with/without magnetic field.

The calculation of the effective Young's Modulus $(\tilde{E})$ of the sample was obtained as described for the alginate beads in section 3.1.2.3. The region to be fitted was chosen to be in the low forces region of the force curve (from $30-45 \mathrm{nN}$ ) to ensure the linear elastic regime. 217.

Several repetitions (data not shown) of indentation experiments were performed on more than 15 chitosan single component hydrogels, showing the same mechano-magnetic coupling in only some cases (due to a higher noise in the measurement before protocol optimisation). Yet technical difficulties on indenting the material, or different experimental environments made these results not robust enough or comparable for statistical analysis 
with the results presented in this thesis; nevertheless confirming them. An example of this is the initial use of a $20 \mu \mathrm{m}$ PE bead instead of the $50 \mu \mathrm{m}$ glass bead attached to the cantilever. The smaller PE bead rendered a higher $\tilde{E}$ dispersion increasing the noise on the observed behaviour due to a smaller indentation area in a very porous and anisotropic material. Similarly, several $\vec{B}$ intensities were used, initially limited to permanent magnets as described above.

Another example is the optimisation of the chitosan concentration. Scaffolds were fabricated in concentrations ranging from $0.5-1.25 \% \mathrm{w} / \mathrm{v}$ and microindented as described above.

In a similar way, initial experiments were not crosslinked with genipin. Instead, in a first attempt, scaffolds were treated (after freeze drying) with a $1 \mathrm{M} \mathrm{NaOH}$ solution for 30 minutes at room temperature in order to avoid scaffold redissolution. This resulted in shrinkage of the foam and higher inhomogeneities. A second approach consisted in suspending a chitosan scaffold (without genipin) after the freeze drying in a $0.23 \mathrm{M}$ genipin solution and placed in the oven at $37^{\circ}$ for 12 hours to crosslink. This resulted in a less homogeneous matrix with higher $\tilde{E}$ dispersion. Lastly, genipin was added to the chitosan solution and homogenised before aliquoting into the molds, freezing and freeze drying as described in the materials and methods.

\subsubsection{Bulk Shear Rheometry mechanical characterisation}

A rheometer with a parallel plate geometry (Anton Paar Rheometer Physica MCR301) with $7.966 \mathrm{~mm}$ plate diameter and a gap of $5 \mathrm{~mm}$ was used. Each sample was placed in a plastic petri dish immersed in 1 x PBS. Data were obtained in presence/absence of a permanent NdFeB magnet (First4magnets UK, B = $180 \mathrm{mT}$ measured at the height of the sample) at room temperature. The magnet was placed below the petri dish and fixed with double-sided tape. For each configuration, the system was calibrated before the 
data acquisition. Frequency sweeps were performed from 0.1 to $1 \mathrm{~Hz}$ at $1 \%$ shear strain amplitude.

5 scaffolds fabricated in different batches (data not shown) were assessed to optimise the experiment and find the optimal gap distance since, if the polymer is compressed too much, no effect was observed. This could show the importance of the electrolyte flow in the magneto-mechanical coupling and give evidence in support of magnetohydrodynamic effects, because when there is no electrolyte flow, the behaviour seems to be stopped.

\subsubsection{Results}

\subsubsection{AFM indentations}

Using the Hertz model to fit $\mathrm{F}$ vs $\delta$ curves (section 4.2.4), values of $\tilde{E}$ were obtained in several positions in each of the samples shown in figure 3.7 (a) and (b). (c) shows typical force vs. indentation curves on chitosan recorded in the same position in the presence (red line) and in the absence (black line) of the magnetic field. The slope of the curves is clearly affected by the magnetic field. To test whether the presence of the magnetic field would induce artefacts, PDMS was used as control. It is apparent that the two curves overlap with no significant change (d). The Hertz Model fits are shown in yellow. Figure 3.7 (e) shows the average obtained for the 100 curves in all the positions in the presence (red) and absence (black) of the external magnetic field. While no change of can be detected for the PDMS, for chitosan the difference between the overall Young's moduli with/without the magnetic field $\Delta E^{*}=E_{B \neq 0}^{*}-E_{B=0}^{*}$ is about $9 \mathrm{kPa}$ (approx. $50 \%$ increase).

\subsubsection{Bulk shear rheometry}

The two components of the bulk dynamic modulus, $G^{\prime}$ and $G^{\prime \prime}$, were obtained for chitosan and the PDMS control using oscillatory shear rheometry in parallel plate geometry as 
a)
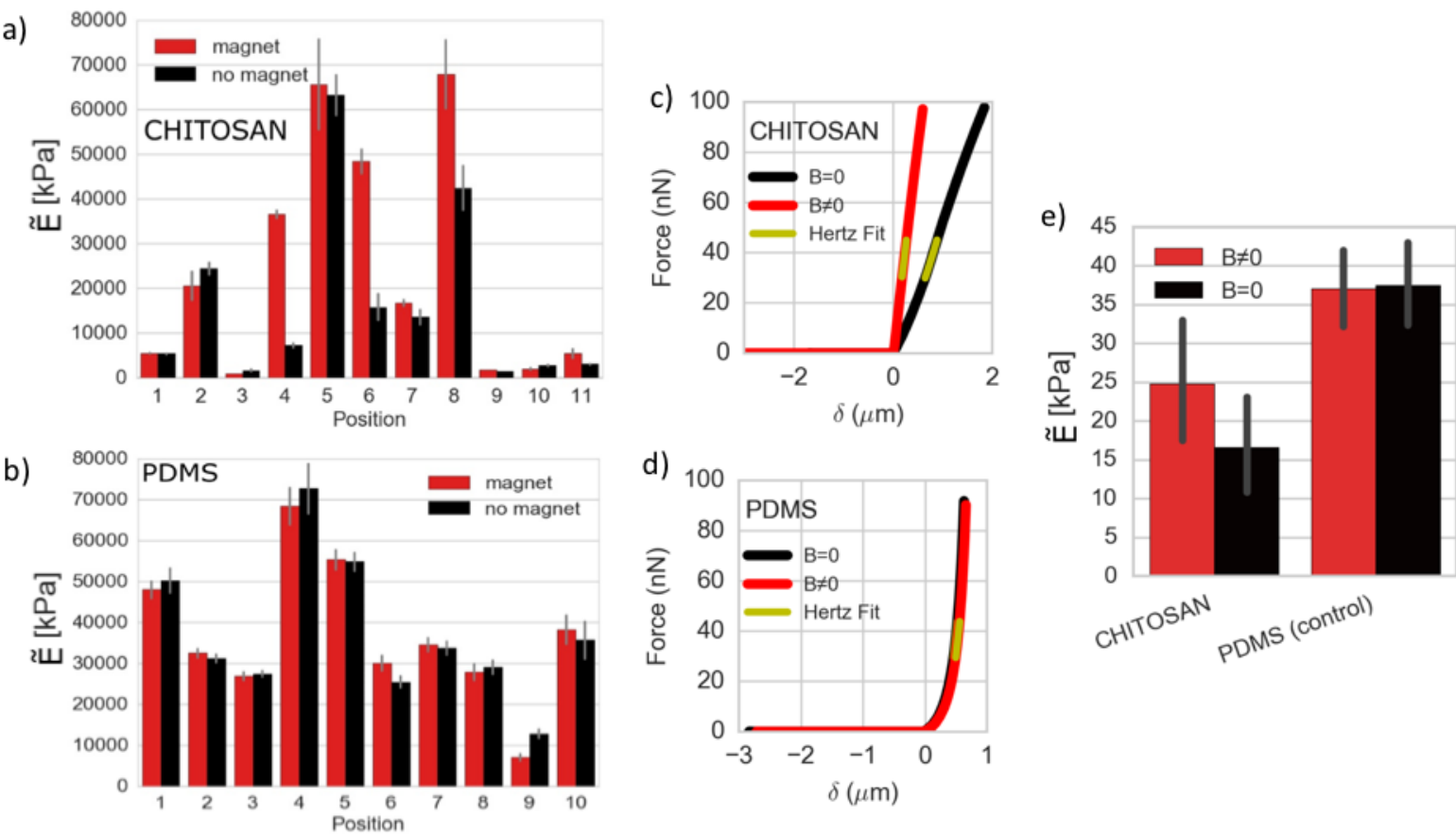

Figure 3.7: Chitosan and PDMS control AFM microindentations. (a) and (b) shows the mean $\tilde{E}$ value of 100 curves recorded at each position in the polymer. (c) $\mathrm{F}$ vs. $\delta$ curves in one position on chitosan, in the presence (red line) and in the absence (black line) of the magnetic field, showing a clear different slope. (d) PDMS F vs. $\delta$ control curves showing no significant change. The Hertz Model fits are shown in yellow. (e) Mean $\tilde{E}$ of chitosan and PDMS comprising all of the indented points. 

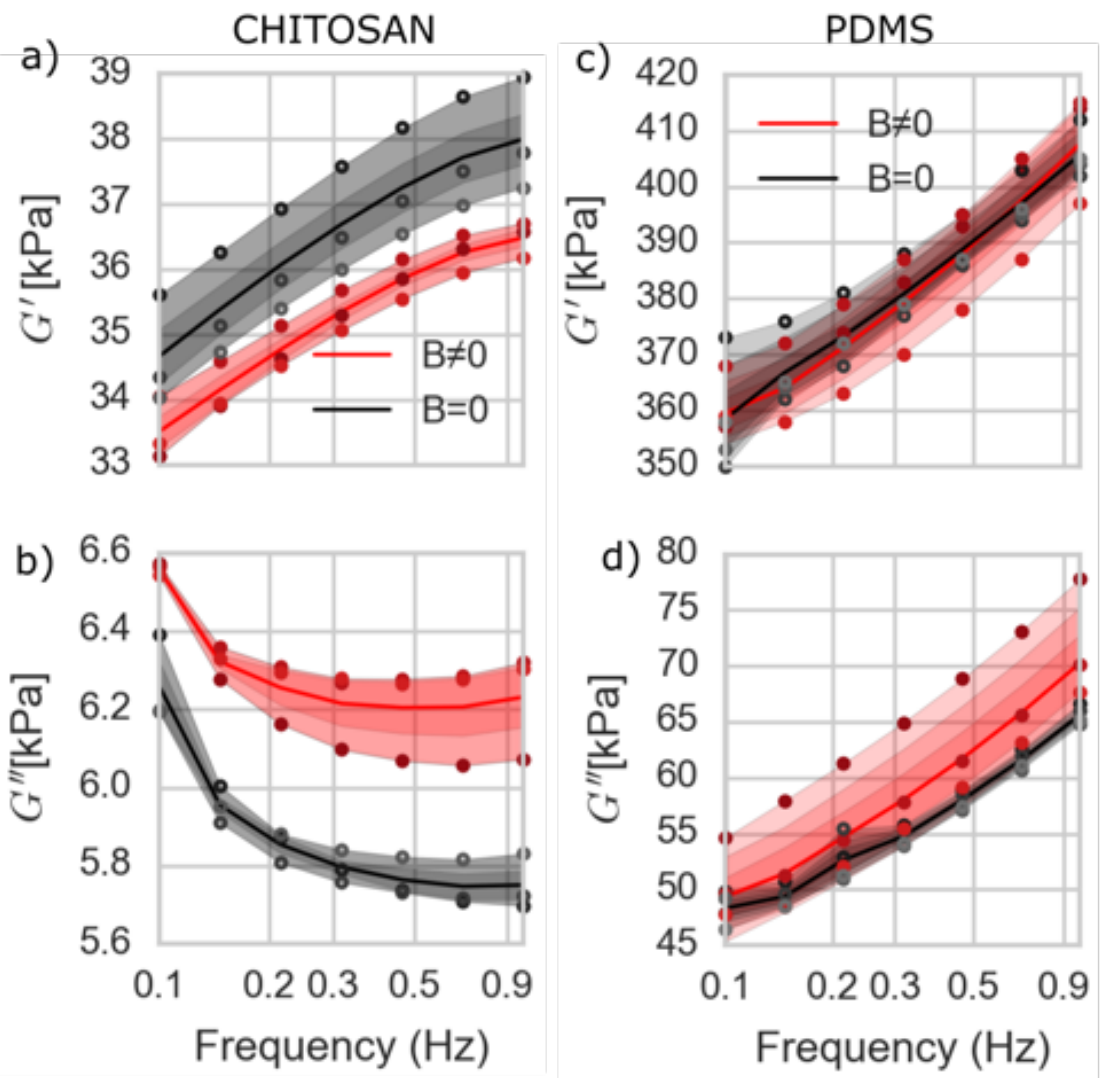

Figure 3.8: Bulk oscillatory shear rheometry experiments on chitosan and PDMS control. Semilog plot of $G^{\prime}$ and $G^{\prime \prime}$ obtained for (a), (b) chitosan and (c), (d) PDMS. The black and red markers correspond to repetitions of frequency sweeps done in the absence and in presence of the magnetic field, respectively. The black and the red lines represent the averages of the different repetitions depicted in the shaded areas. 
explained in experimental techniques, chapter 2. Fig. 3.8 (a-d) shows data obtained from several independent frequency sweeps in the absence (black empty markers) and presence (red filled markers) of an external $\vec{B}$. The respective mean value of $G^{\prime}$ and $G^{\prime \prime}$ is plotted with continuous lines in the range of frequencies studied. Figure 3.8 (a), (b) shows an evident decrease in $G^{\prime}$ and increase in $G^{\prime \prime}$ in the chitosan sample when the $\vec{B}$ is introduced. In contrast, the PDMS control does not present any significant difference (see fig. 3.8 (c), (d)).

\subsubsection{Discussion}

In this second part of the chapter, I have described the fabrication and characterisation of the mechanical properties of chitosan hydrogel locally and in bulk in the presence and absence of small magnetic fields. The most important result is that the presence of $\vec{B}$, affects the mechanical properties although AFM and rheometry results differ, in AFM the average $\tilde{E}$ increases whereas the elastic, shear $G^{\prime}$ decreases in the rheometry measurement.

\subsubsection{AFM indentations}

Reports on mechanical characterisation of chitosan constructs using AFM are scarce. Yet several approaches have been reported; Yao et. al [245] report the fabrication and characterisation via AFM nanoindentations of chitosan/gelatin ( $1 \%$ and $0.66 \% \mathrm{w} / \mathrm{v}$, respectively) hybrid hydrogels. They fabricated low-porosity thin films and then assessed the swelling ratio and increment in porosity, compared to change in $\mathrm{E}$ by indenting at different times and at $\mathrm{pH} 1.2$ and 12. They observed a decrease in E due to an increase in swelling and a maximum $\mathrm{E}$ of $1.2 \mathrm{MPa}$, lowering down to $0.57 \mathrm{MPa}$ (after $29 \mathrm{~min}$ ) at $\mathrm{pH} 12$ and $0.48-0.14 \mathrm{MPa}$ at $\mathrm{pH} 1.25$. These values are one order of magnitude larger than the ones presented in this thesis (considering a $\nu=0.5$ to calculate $\mathrm{E}$ from reported $\tilde{E}$ ). The fabrication protocol by Yao involves double the concentration of chitosan, more importantly, 
the films are not as porous as our hydrogels which is likely to be the main reason for the difference in E.

Similarly, non-porous thin films of chitosan for bone TE are reported 133, 246]. Wang et. al fabricated films of $1 \% \mathrm{w} / \mathrm{v}$ high MW chitosan and chitosan-MWCNTs (Multi-way carbon nanotubes) nanocomposite and characterised them mechanically by nanoindentation with MTS Nano Indenter XP, reporting an E of 1.080.04 GPa for chitosan single component films and 2.150.09 GPa for 2\% MWCNTs composites 133. Furthermore, Verma et. al 246 fabricated and mechanically characterised by AFM nanoindentations chitosannanohydroxyapatite (Ch-HPA) and chitosan-polygalacturonic-nanohydroxyapatite (ChPA-HPA) composite thin films. They report an E of $1.02 \mathrm{GPa}$ (Ch-HPA) and 2.06 GPa (Ch-PA-HPA). In these cases, the reported $\mathrm{E}$ is much larger, again mainly due to lack of porosity and the fabrication of composites which in this case is even more pronounced than at the reported by Yao et. al.

\subsubsection{Bulk shear rheometry}

Moura et. all assessed the effect of genipin crosslinker on the rheological properties of chitosan hydrogels. They report the $G^{\prime}$ and $G^{\prime \prime}$ of hydrogels fabricated with different concentrations of the crosslinker genepin and a $G^{\prime}$ from 0.01 to $100 \mathrm{~Hz}$. For the highest genipin concentration $(0.2 \% \mathrm{w} / \mathrm{v})$, they obtained a $G^{\prime}$ of $1 \mathrm{kPa}$ at $0.1 \mathrm{~Hz}$ 247]. In this thesis, we report a $G^{\prime}$ of $34.660 .67 \mathrm{kPa}$ in the absence of the field. There is a difference of one order of magnitude between the $G^{\prime}$ reported by Moura et. all and our value, that can be expected because of a higher chitosan crosslinking ratio used in this thesis due to a much higher concentration of genepin (5.2\%). 


\subsubsection{Magneto-mechanical coupling of hydrogels}

Our results show that the presence of a $\vec{B}$, in shear rheometry results in an increase of the shear $G^{\prime \prime}$ and decrease of the shear elastic modulus $G^{\prime}$ and an increment of the normal $\tilde{E}$ measured by AFM. The most striking result is the dependence of the mechanical properties on the magnetic field which is an unexpected result. Due to the anisotropic nature of the sample, the obtained values and behaviours of the Chitosan from AFM microindentation and $G^{\prime}-G^{\prime \prime}$ from oscillatory shear rheometry in the presence and absence of the field, cannot be directly compared. First, because of the size difference, since AFM probes the microscale and shear rheometry probes the bulk properties. Furthermore, AFM $\tilde{E}$ describes de normal component of the modulus (AFM applies a normal force) and rheometry yields the shear modules, obtained by a time dependent shear strain applied by a rotating plate in contact with the sample. The differences in the results obtained by both techniques point towards the possible origin of the magneto mechanical coupling as discussed below.

It is feasible that the deformation of the polymer chains results in electrical charges flow or dipole deformations that can be affected by the presence of $\vec{B}$; this is particularly plausible because chitosan has been previously reported to exhibit piezoelectric behaviour [248]. Since a deformation can polarise the material, it is conceivable that electrically conductive water and maybe even electrolytes from the PBS medium can flow through the porous hydrogel creating a current upon mechanical deformation.

Apart from the effect of the magnetic field on the piezoelectric properties of the materials it is also possible that the movement of fluid in the porous material produces and/or enhances this process. It is known since the 1940s that if an electrically conducting fluid moves in a magnetic field, electric fields are induced in it and electrical currents flow [249]. The magnetic field exerts forces on these currents which may considerably modify the flow [250]. This phenomenon is known as magnetohydronynamics and is currently used i.e. in applications in microfluidics 251] and even has been shown to enhance the survival of tis- 
sues ex vivo 252$]$. In our set up, a $\vec{B}$ would be able to produce a Lorentz force on the fluid [253], which, in turn, would be able to affect the mechanical properties of the hydrogel. This hypothesis is depicted in Figure 3.9 and calculations on ionic concentrations in the buffer that produce a Lorentz force are shown in appendix A. The results in app A were compared to studies by West et. al [254] who fabricated continuous flow microreactors using an electrolyte (500 mM KCL) with an ionic concentration in the same order of magnitude $\left(10^{17}\right.$ if calculated as described in app A considering a volume of $\left.1 \mathrm{~mm}^{3}\right)$ to the 1xPBS electrolyte used in this thesis.

Fig. 3.9 (a) shows the resultant electrolyte solution flow because of the application of a normal force with the AFM cantilever and a bead attached to the tip. (b) The presence of a $\vec{B}$ on a moving conductive fluid should induce electrical currents that would further polarise the material, changing the polymeric chains, the flow of electrolite and the mechanical properties of the hydrogel.

\subsubsection{Conclusions}

Chitosan hydrogels have been extensively used in biomedical applications as well as for TE as 3D scaffolds to seed cells because of its biodegradablility, biocompatibility and its hypoallergenic, antibacterial and bioadhesive characteristics.

AFM indentation experiments show a normal stiffening of the hydrogel by a mean reduced modulus $\tilde{E}$ increase of approx. $50 \%$ in the presence of a $45 \mathrm{mT}$ field. Similarly, we have found significant bulk decreases in the storage modulus $G^{\prime}$ (solid-like contributions of the stress response) and increase in loss modulus $G^{\prime \prime}$ (fluid-like response) measured by shear rheometry in the presence of a $180 \mathrm{mT}$ field. This behaviour could be due to a combination of material responses, including magnetohydrodynamic effect as proposed in Fig. 3.9 .

PDMS controls were used to assess a systematic errors due to the experimental set up, yet other experiments using porous neutral polymers would be useful to assess the influence 
a)

$$
\overrightarrow{\mathrm{B}}=0
$$

Force $(F) \rightarrow$ Polymer deformation $\rightarrow$ Electrolite flow

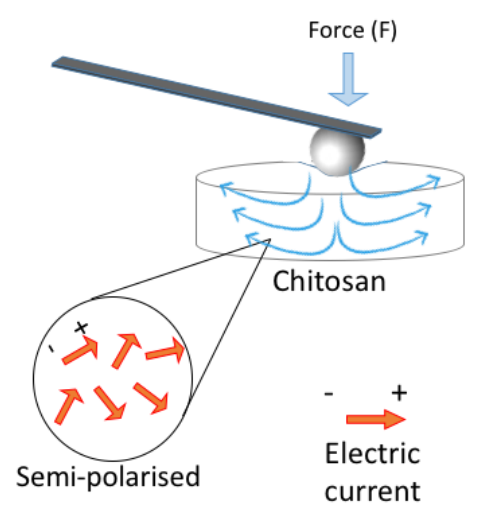

(I) b) $\quad \overrightarrow{\mathrm{B}} \neq 0$

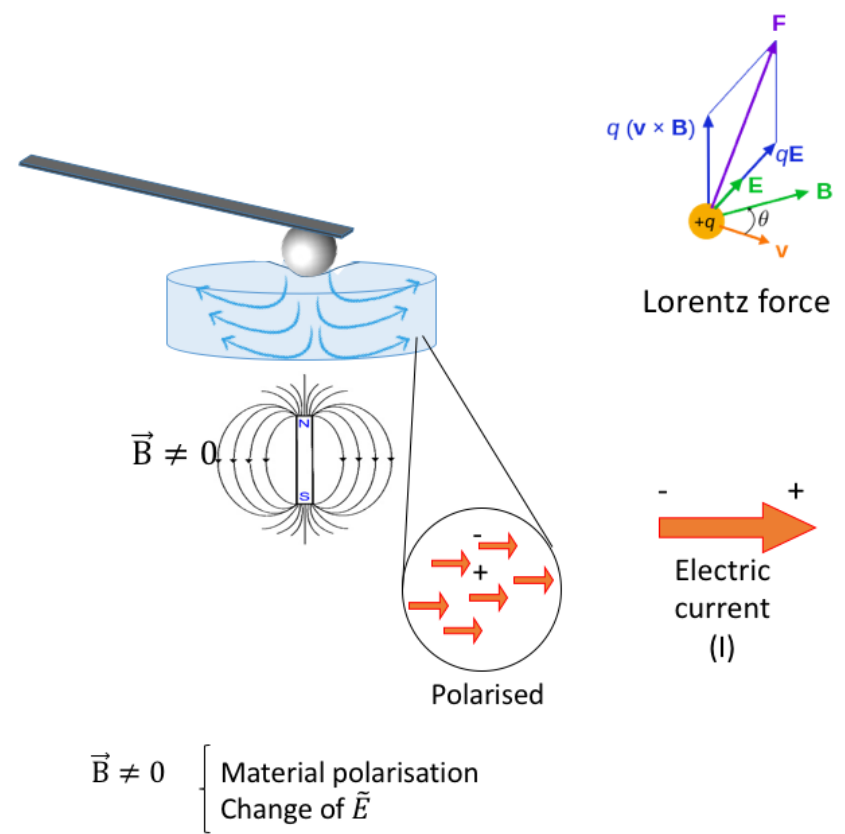

Figure 3.9: Schematic representation of the magnetohydrodynamic effect resultant from an applied normal force to a chitosan hydrogel. (a) In the absence of the $\vec{B}$, the applied normal force deforms the polymeric mesh and creates an electrolite flow which might polarise the hydrogel to a certain degree. (b) Once the $\vec{B} \neq 0$ and given medium flow, a Lorentz force is produced that further polarises the material, changing the $\tilde{E}$ of the hydrogel. 
on the behaviour due to the flow of the electrolyte within a non-polarisable mesh.

Similarly, a comparison of the different electrolytes could be assessed to see a possible change in behaviour as well as a quantification and change of concentration and crosslinking ratio of chitosan in order to study the polarisation of the hydrogel that could be due to the presence of charged amino groups in the chain, which would make the charge density of chitosan to be dependent on $\mathrm{pH}\left(\mathrm{NH}^{+3}\right.$ protonation in acidic conditions).

Our results demonstrate, for the first time, a mechano-magnetic coupling of a natural polymeric hydrogel and a change in its mechanical properties due to the presence of weak magnetic fields. This could potentially have an impact on future applications of chitosan hydrogels. 


\section{Chapter 4}

\section{Smart nanocomposite hydrogel for tissue engineering}

The work presented in this chapter is being prepared for publication in joint co-authorship with Alba Piacenti.

As described in the introduction in section 1.1.2.3, nanocomposite materials have extensively been used in biomedical applications because of their higher mechanical strength and capacity to respond to external stimuli.

In this chapter, first we describe the synthesis and structural/morphological characterisation of $\mathrm{Fe}_{3} \mathrm{O}_{4}$ nanowires. Then we describe the use of these nanowires in magnetically responsive chitosan nanocomposite hydrogel.

Furthermore, by a combination of local AFM microinentation and bulk microrhoreology, we demonstrate that the mechanical properties of this material can be modified in the presence of small magnetic fields. 


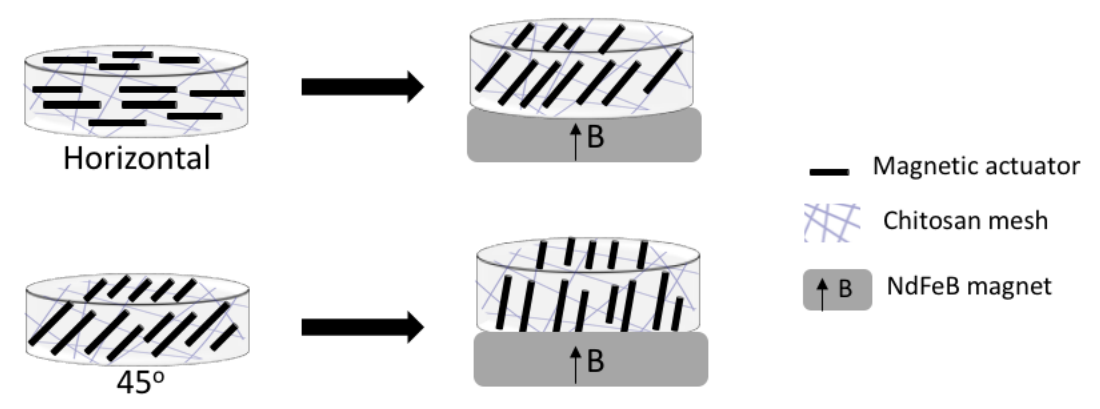

Figure 4.1: Schematic representation of alignment of nanowires embedded in a chitosan polymeric mesh and the realignment in the presence of a NdFeB magnet.

\subsection{Introduction}

Polymeric composites fabricated with nanostructures have attracted great interest given their synergy-derived features, offering the possibilities of unique mechanical, optical, electrical, thermal or magnetic properties, due to the interaction of the components and cooperative individual properties. These are easily tunable by the adjustment of concentration ratios and individual characteristics.

Furthermore, these materials are designed to respond to environmental variables. A preferred method of actuation is the use of magnetic fields which deliver a better performance and higher forces that other manipulation strategies such as electric fields, optical methods or acoustic waves (including ultrasound) [100]. Additionally, magnetic fields are not invasive, biologically inert, and can be safely used in aqueous environments.

As described previously in chapter (3), the current technological route to add magnetic responsiveness to a natural polymer is by incorporating micron and nano-sized magnetisable particles.

In this chapter, we describe the fabrication of a magnetically responsive nanocomposite material comprising nanowires embedded in a chitosan polymeric lattice. The $\mathrm{Fe}_{3} \mathrm{O}_{4}$ nanowires are aligned in different configurations from fabrication as shown in figure 4.1 .

The hypothesis is that if the nanowires are subject to a magnetic field $(\vec{B})$ in a certain 
direction during fabrication (horizontal or $45^{\circ}$ ), their orientation will not be random. The further realignment in a different direction using an external $\vec{B}$ will create tension and modify the mechanical properties of the material. Furthermore, the ability to actuate the scaffold could modify pore size to i.e. induce cellular internalisation, or modulate nutrient and oxygen transport.

Additionally, being able to change the mechanical properties of the scaffold by using magnetic fields might be useful to induce cellular differentiation. Mesenchymal stem cells (MSCs) are multipotent stromal cells that can differentiate into a variety of cell types, such as osteoblasts, chondrocytes, myocytes and adipocytes. MSCs have been shown to specify lineage and commit to cellular phenotypes with extreme sensitivity to the mechanical properties and Young's modulus of elasticity of the substrate at the tissue-level [40].

Because of this, in this chapter we describe the study of the mechanical characterisation of bulk and local properties by shear rheometry and atomic force microscopy (AFM)-based indentations, respectively in order to characterise the mechano-magnetic response of these nanocomposite materials by weak magnetic fields below $0.2 \mathrm{~T}$.

\subsection{1 $\quad \mathrm{Fe}_{3} \mathrm{O}_{4}$ in biomedical research}

$\mathrm{Fe}_{3} \mathrm{O}_{4}$ or magnetite nanoparticles have been extensively used as a magnetisable actuators due to their unique properties, such as low toxicity, superparamagnetism, high surface area, large surface-to-volume ratio and easy separation under external magnetic fields [255]. Their magnetic responsiveness, allows them to orient to an external $\vec{B}$ on their easy axis.

More specifically, $\mathrm{Fe}_{3} \mathrm{O}_{4}$ nanoparticles have been used as sensors 256 258, as contrast agents in MRI studies 259], in magnetic hyperthermia 260, 261] and in drug delivery applications (and potential TE applications) where chitosan/ $\mathrm{Fe}_{3} \mathrm{O}_{4}$ nanoparticles were subject to cell proliferation assays, successfully showing composite biocompatibility [262].

Furthermore, $\mathrm{Fe}_{3} \mathrm{O}_{4}$ nanowires have also been preferred over nanoparticles because of 
their shape anisotropy, and their ability to generate higher torque due to magnetisation in an elongated or long easy axis in applications such as drug delivery [98, flow 263 and tactile 264 sensors, hyperthermia 265 and other applications as magnetically driven mechanical cancerous cell death 266.

\subsection{Materials and methods}

\subsubsection{Synthesis of $\mathrm{Fe}_{3} \mathrm{O}_{4}$ nanowires}

$\mathrm{Fe}_{3} \mathrm{O}_{4}$ nanowires were prepared by hydrolysis of $\mathrm{Fe}^{2+}$ and $\mathrm{Fe}^{3+}$ according to [267].

An initial protocol was implemented via a reflux reaction of a solution of $420 \mathrm{mM}$ $\mathrm{FeCl}_{3} \cdot 4 \mathrm{H}_{2} \mathrm{O}, 210 \mathrm{mM} \mathrm{FeSO} 4 \cdot 7 \mathrm{H}_{2} \mathrm{O}$ and $1 \mathrm{M}\left(\mathrm{NH}_{2}\right)_{2} \mathrm{CO}$ prepared with deoxygenated ultrapure (MillliQ) water. The reaction was performed in an oil bath at $90-95^{\circ} \mathrm{C}$ for 12 hours followed by ageing for $12 \mathrm{~h}$. The solution changed from yellow to black during the reaction indicating the formation of magnetite. The product was washed 4 times with pure, deoxygenated water and decanted with a magnet to keep the magnetic product, followed by freeze drying at $-80^{\circ} \mathrm{C}$ for 24 hours.

The synthesis of $\mathrm{Fe}_{3} \mathrm{O}_{4}$ nanowires followed the next set of reactions:

$$
\begin{gathered}
\left(\mathrm{NH}_{2}\right)_{2} \mathrm{CO}+\mathrm{H}_{2} \mathrm{O} \longrightarrow 2 \mathrm{NH}_{3}+\mathrm{CO}_{2} \\
\mathrm{NH}_{3}+\mathrm{H}_{2} \mathrm{O} \longrightarrow \mathrm{NH}_{4}^{+}+\mathrm{OH}^{-} \\
\mathrm{Fe}^{3+}+3 \mathrm{OH}^{-} \longrightarrow \mathrm{Fe}(\mathrm{OH})_{3} \longrightarrow \mathrm{FeOOH}+\mathrm{H}_{2} \mathrm{O} \\
\mathrm{Fe}^{2+}+2 \mathrm{OH}^{-} \longrightarrow \mathrm{Fe}(\mathrm{OH})_{2} \\
2 \mathrm{FeOOH}+\mathrm{Fe}(\mathrm{OH})_{2} \longrightarrow \mathrm{Fe}_{3} \mathrm{O}_{4}+2 \mathrm{H}_{2} \mathrm{O}
\end{gathered}
$$


Further morphological characterisation (described later in section 4.3.1.1, figure 4.3) showed a bifurcation on the resulting nanowires. In order to obtain single crystal nanowires, a second protocol was implemented following the previously described reaction but using a Teflon flask, placed in a autoclave reactor with a $0.45 \mathrm{~T}$ temperature resistant $\mathrm{NdFeB}$ magnet (first4magnets) in the bottom to induce nanowire growth on the easy axis [140]. The reactor was placed in an oven at $130^{\circ} \mathrm{C}$ for 6 hours. After this time, the reactor was allowed to cool down at room temperature overnight. The resulting black magnetic dispersion was washed and dried as described above. Several batches of nanowires were prepared, resulting in a slight demagnetisation of the magnet used, which caused the formation of not only nanowires, but also spherical magnetic aggregates of $\mathrm{Fe}_{3} \mathrm{O}_{4}$ as expected and described by Wang et. all 140 (this is further discussed in section 6.2.1).

\subsubsection{Chitosan scaffolds fabrication}

Scaffolds of chitosan single component and chitosan containing $\mathrm{Fe}_{3} \mathrm{O}_{4}$ nanowires were prepared by freeze drying as shown in figure 4.2 and as described previously in chapter 3 , section 3.2.2.1. Chitosan $/ \mathrm{Fe}_{3} \mathrm{O}_{4}$ nanocomposites, were prepared by adding $0.1 \% \mathrm{w} / \mathrm{v} \mathrm{Fe}_{3} \mathrm{O}_{4}$ nanowires synthesised before (described in 4.2.1) to the chitosan single component solution and dispersed in a water sonication bath for 10 seconds plus vortexing.

All dispersions were frozen in $1.3 \mathrm{~cm}$ diameter molds at $-80^{\circ} \mathrm{C}$ for 30 minutes. Nanocomposites were frozen in the presence of a $180 \mathrm{mT} \mathrm{NdFeB} \mathrm{magnet} \mathrm{at} \mathrm{different} \mathrm{ori-}$ entations (horizontal, $45^{\circ}$ and not-oriented) as shown in figure 4.2 in the lower left corner. The matrices were freeze dried and then crosslinked as described previously 3.2.2.1 and depicted in figure 4.2 . 


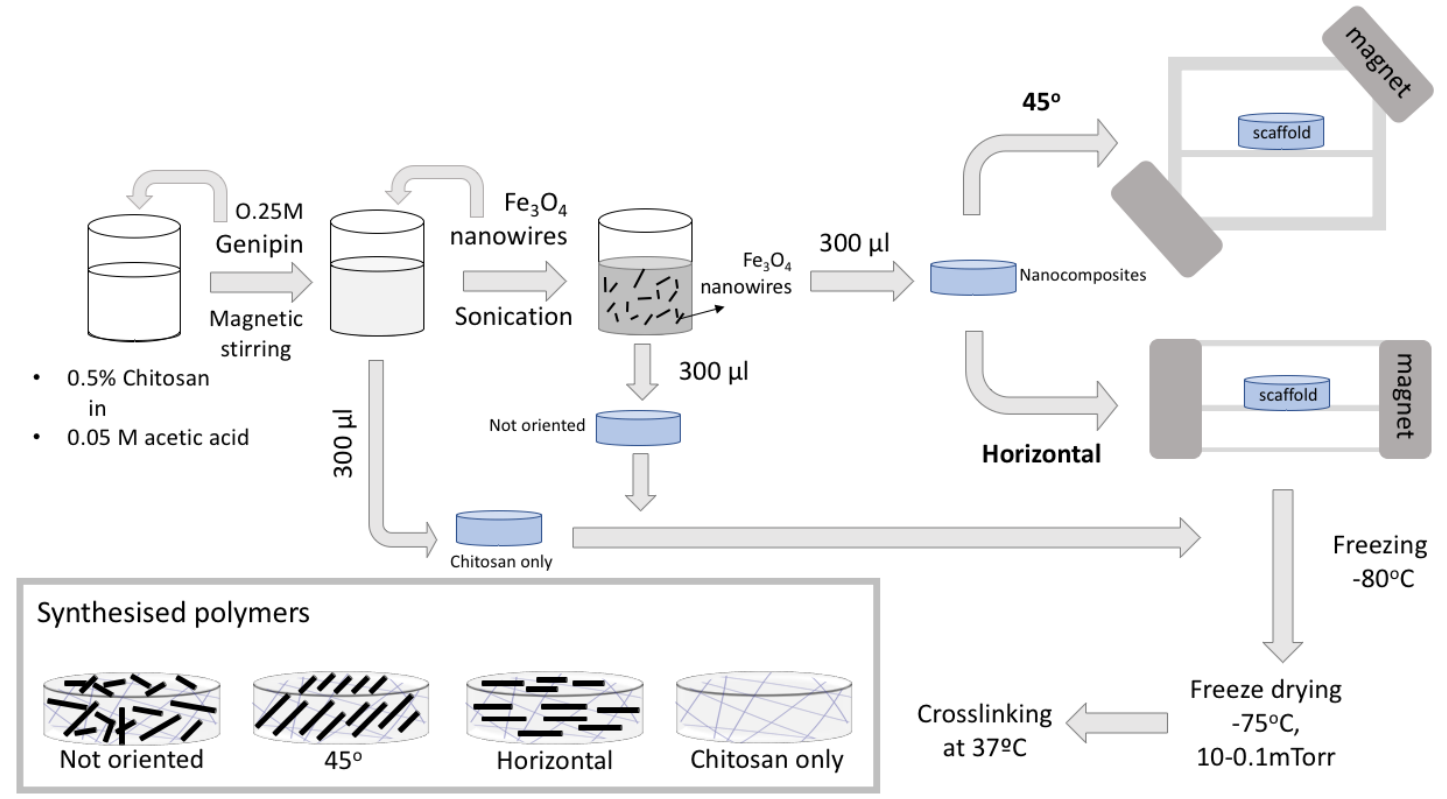

Figure 4.2: Chitosan single component and chitosan $/ \mathrm{Fe}_{3} \mathrm{O}_{4}$ nanocomposite scaffold preparation process. Nanowires were subjected to a $\vec{B}$. Scaffolds were frozen at $-80^{\circ} \mathrm{C}$ in the presence of a $180 \mathrm{mT} \vec{B}$ at different orientations ( $45^{\circ}$ and Horizontal). Scaffolds were then freeze dried for 30 hours and then crosslinked at $37^{\circ} \mathrm{C}$ for 24 hours. Bottom left corner shows the schematic representation of the fabricated polymers

\subsubsection{Structural characterisation}

AFM images of the nanowires and scaffolds were obtained using a commercial MFP-3D from Asylum Research (Santa. Barbara, California) using a OMCL-AC240TS cantilever (Olympus). Nanowires were dispersed on a mica surface.

The microstructure of the nanowires were analysed by TEM on a FEI Talos F200X instrument operated at $200 \mathrm{keV}$ acceleration voltage. Both, TEM and scanning TEM (STEM) operation modes of the instrument were used for the study. STEM studies were performed in the high angle annular dark field (HAADF STEM) imaging mode and accompanied by elemental content analysis using energy dispersive spectroscopy EDX STEM. STEM and EDX STEM studies were carried out using a $0.8 \mathrm{~nm}$ probe size.

SEM images of nanowires in figure 4.3 were acquired by a secondary electron detector in Zeiss ULTRA 55. The sample was dispersed on a Si wafer and dried. 
SEM images of dry chitosan scaffolds were obtained using a FEI QUANTA 600 F. The foam like scaffolds were stuck to the SEM holder using carbon tape by flicking them upside down after crosslinking in the oven.

\subsubsection{Local mechanical characterisation}

AFM- based microindentations and the calculation of the effective Young's Modulus were performed for horizontal, not-oriented and $45^{\circ}$ configurations as described previously in chapter 3, subsection 3.2 .2 .2 . The region to be fitted was chosen to be in the low forces region of the force curve (from $30-45 \mathrm{nN}$ ) to ensure the linear elastic regime. [217].

These experiments were performed in more than 30 different scaffolds as described in chapter 3, section 3.2.2.2. Furthermore and as discussed before, chitosan concentrations were varied from $0.5-2 \% \mathrm{w} / \mathrm{v}$ and $\mathrm{Fe}_{3} \mathrm{O}_{4}$ nanowires from $0.05-0.5 \% \mathrm{w} / \mathrm{v}$, observing an enhanced behaviour at the concentrations described in the materials and methods section.

\subsubsection{Bulk Shear Rheometry mechanical characterisation}

Rheometry experiments were performed as described in chapter 3 , section 3.2.2.3 for all nanocomposite configurations (horizontal, not-oriented and $45^{\circ}$ ).

More than 10 hydrogels were assessed (data not shown) as described in chapter 3 . section 3.2 .2 .3 .

We performed frequency sweeps from 0.1 to $1 \mathrm{~Hz}$ to describe time-dependent behaviours of the samples with a strain amplitude $\gamma_{A}$ constant at $1 \%$. 


\subsection{Results}

\subsubsection{Morphological characterisation}

\subsubsection{1 $\quad \mathrm{Fe}_{3} \mathrm{O}_{4}$ Nanowires}

Figure 4.3 (a) shows an AFM image of the synthesised nanowires in the initial reflux reaction. The bifurcation in one nanowire suggests a polycrystalline material that induced the growth of the crystal in more than one direction. This could result in a not energetically favourable direction of spontaneous magnetisation, given the lack of an easy axis of magnetisation to align the nanowires, making it problematic to align these nanowires to

an external $\vec{B}$. Because of this, the second protocol was implemented using a permanent magnet during the synthesis to direct crystal growth as described in the materials and methods. Figure 4.3 (b) and (d) shows AFM images of these nanowires, (c) shows a TEM image and (e) a SEM image.

Figure 4.4 shows the elemental analysis of the synthesised $\mathrm{Fe}_{3} \mathrm{O}_{4}$ nanowires when embedded on a PCL microparticle(described in next chapter 5); (a) and (b) show the nanowire cluster that was analysed and the Fe and $\mathrm{O}$ elements distribution. (c) confirms the presence of $\mathrm{Fe}$ with peaks at 0.7174 and $6.3967 \mathrm{keV}$ and $\mathrm{O}$ at $0.5274 \mathrm{keV}$. The presence of $\mathrm{C}$ from the PCL polymeric lattice is also present at $0.2674 \mathrm{keV}$. The Si substrate at 1.7373 $\mathrm{keV}$.

\subsubsection{Chitosan single component and chitosan nanocomposite morphological characterisation}

Figure 4.5 shows SEM images of the fabricated scaffolds in the presence of a $\vec{B}$ in different configurations as schematised in figure 4.2. Chitosan single component images (figure 4.5 (a) shows the typical morphology of freeze dried chitosan scaffolds. Figure 4.5 (b) shows the not oriented nanocomposite (fabricated without a $\vec{B}$ ), (c) shows the $45^{\circ}$ configuration, 

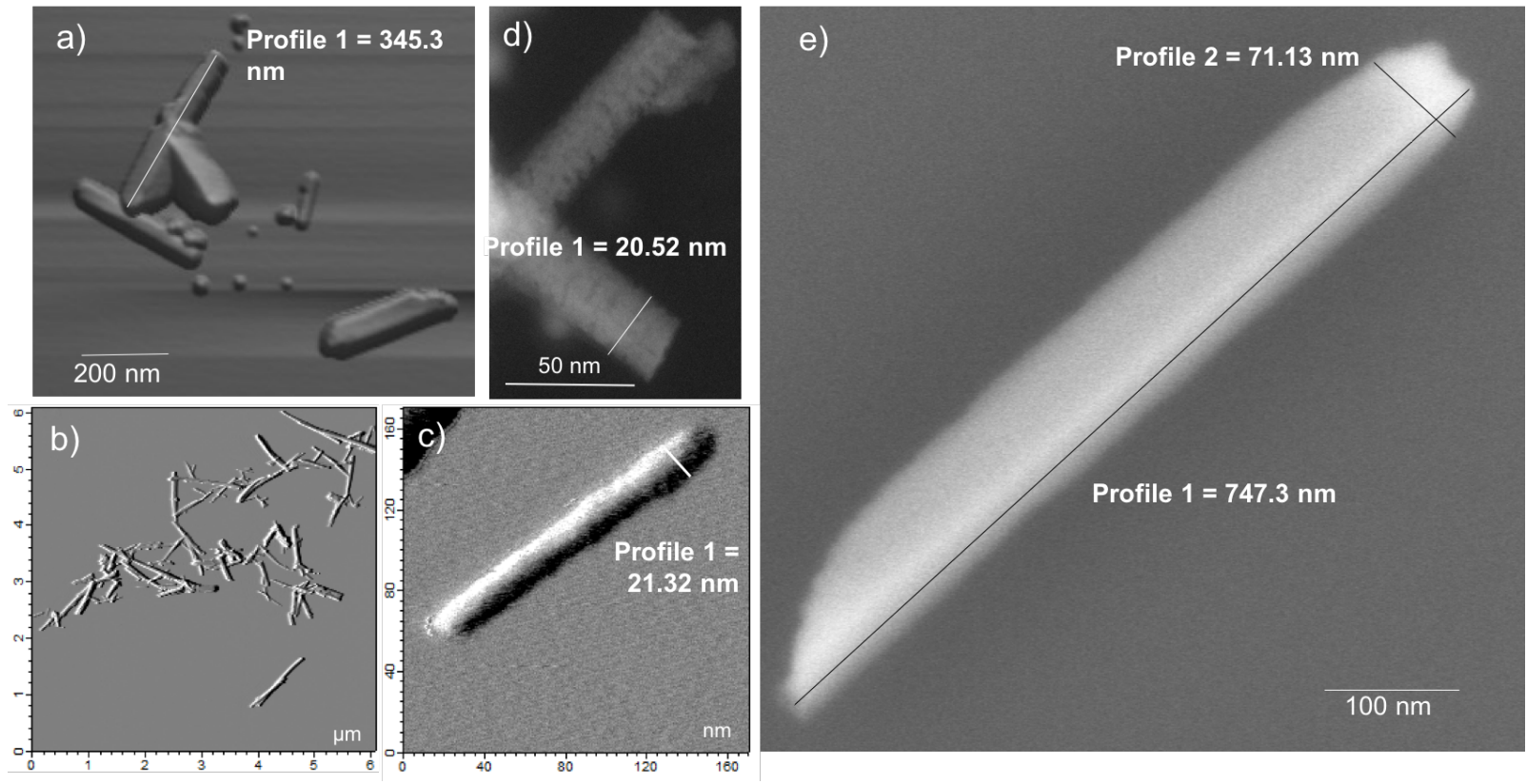

Figure 4.3: Nanowire AFM, TEM and SEM morphology characterisation. (a) AFM image of a branched nanowire synthesised in $\vec{B}=0$. (b, c) AFM images of nanowires synthesised as described in section 4.2 .1 , with a $\vec{B} \neq 0$. (d) TEM image of the nanowires and (e) SEM image of a single nanowire showing the length and width measurements. 

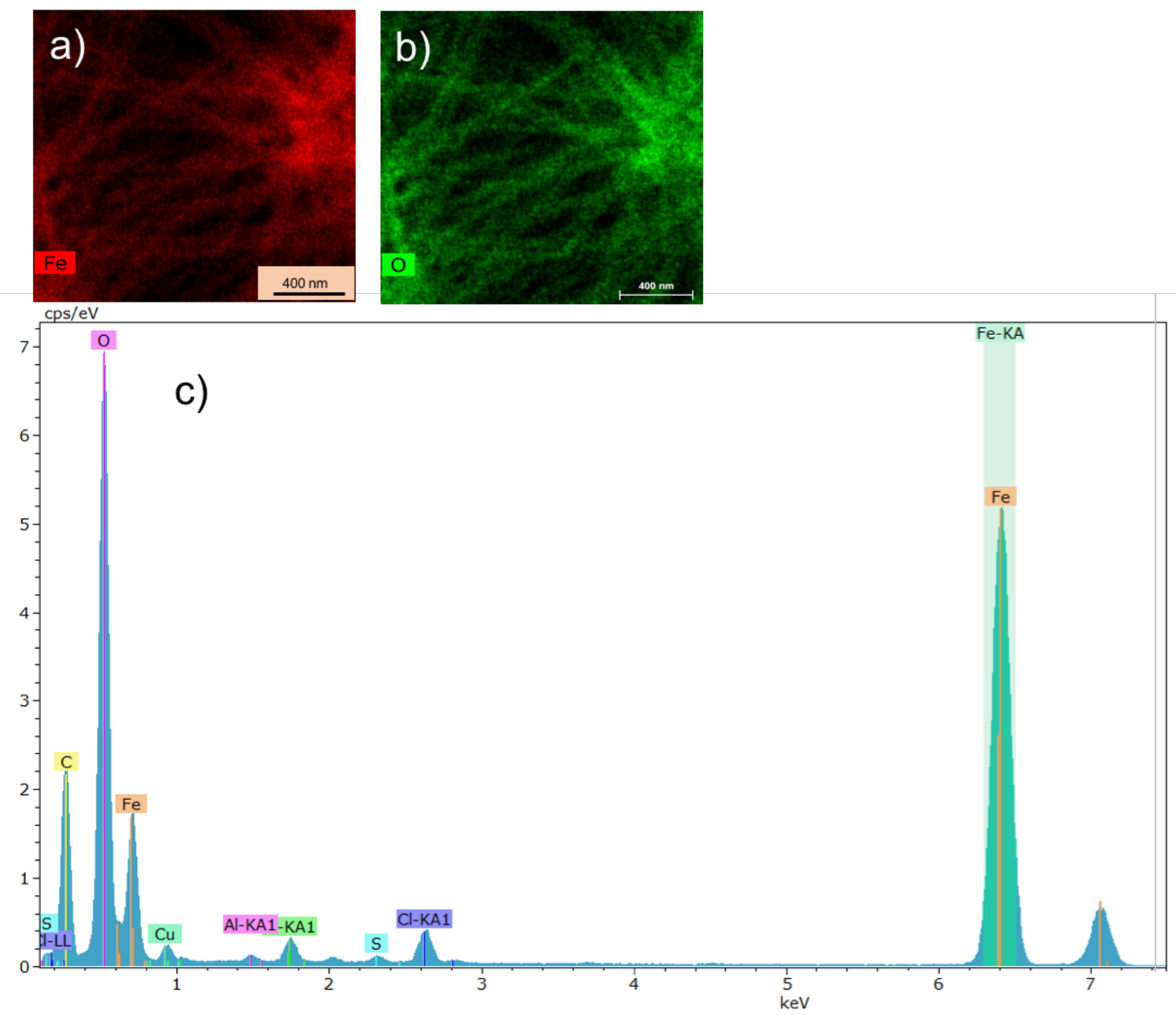

Figure 4.4: EDX analysis of synthesised nanowires.

(a) and (b) $\mathrm{Fe}_{3} \mathrm{O}_{4}$ nanowires embedded within the polymeric PCL lattice showing the $\mathrm{Fe}$ (red) and Oxygen (green) elements present and (c) EDX analysis confirming Fe (0.7174 and $6.3967 \mathrm{keV}), \mathrm{O}(0.5274 \mathrm{keV})$ and $\mathrm{C}$ from the polymeric lattice $(0.2674 \mathrm{keV})$ 
(d-f) show the horizontal configuration. The red arrows show aligned clusters of nanowires in the surface of the polymer and white arrows show the direction of alignment of superficial nanowires.

If we compare the chitosan single component scaffold (a) with any of the nanocomposites (b-f), a different pore size is apparent due to chitosan interactions with Fe from the nanowires. Chitosan is known to be a good chelating agent and forms complexes with Fe, due to the presence of $-\mathrm{NH}_{2}$ groups 268 of the polymeric chain.

It is interesting to note that the direction of alignment in fig. 4.5 (c) for the case of the $45^{\circ}$ configuration in superficial nanowires. The wires agglomerate and assemble in parallel lines. In the case of the horizontal configuration fig. 4.5(d), superficial nanowires are not so apparent. This maybe due to the lack of nanowires being deposited at the bottom of the mold before freezing, yet, we can see a certain direction of alignment in fig. 4.5 (e) and a big cluster of nanowires in (f). This kind of superficial inhomogeneity could be the responsible for such a big dispersion in the measured $\tilde{E}$.

\subsubsection{Mechano-magnetic scaffold characterisation}

\subsubsection{AFM microindentations}

The results obtained from AFM-based indentations are shown in figure 4.6. Microindentations were performed 100 times in each polymer at different positions. Using the Hertz model to fit $\mathrm{F}$ vs $\delta$ curves (see materials 4.2.4), values of $\tilde{E}$ were obtained and an average value of these 100 curves for each position are shown in (a), (b) and (c) for horizontal, $45^{\circ}$ and not-oriented configurations respectively. (d) shows the average $\mathrm{E}^{*}$ value obtained for each polymer.

It is important to note that Chitosan single component has the lowest $\mathrm{E}^{*}$ modulus. This is an expected behaviour since nanostructures in nanocomposite materials are known 

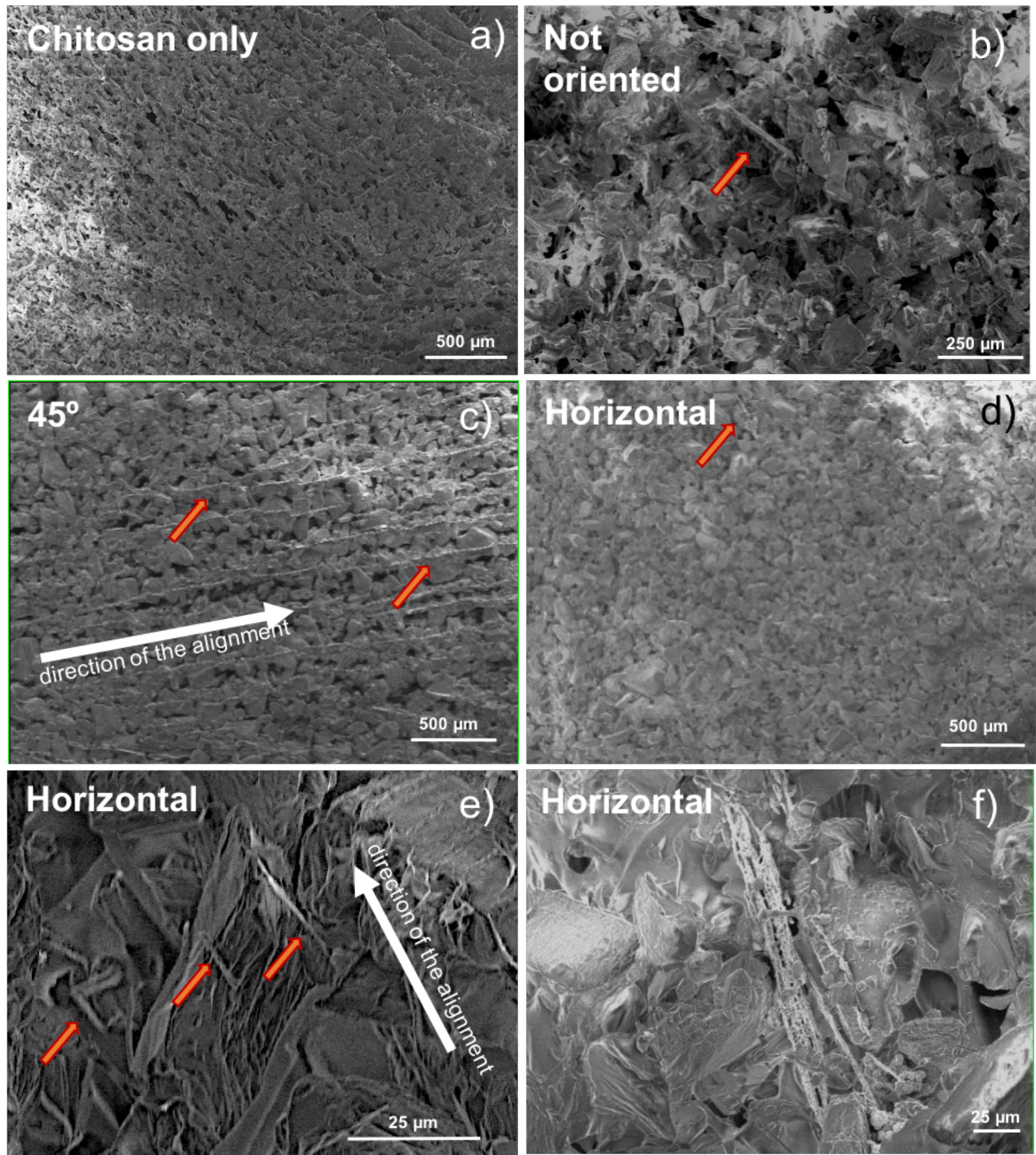

Figure 4.5: SEM images of (a) chitosan single component, (b) not oriented, (c) $45^{\circ}$ and (d-f) horizontal, taken from the bottom of the foam (see materials and methods). White arrows show the direction of alignment and red arrows show aligned clusters of nanowires in the surface of the polymer. 
to reinforce the material and increase their mechanical strength.

If we consider the average between all of the indented positions around each polymer, the difference between the measured $\tilde{E}$ in the presence and absence of the $\vec{B}$ is not significant for the cases of the nanocomposites with higher degree of orientation due to the $\vec{B}$ present during fabrication (horizontal and $45^{\circ}$ ). On the other hand, for the not-oriented $\mathrm{Fe}_{3} \mathrm{O}_{4}$ nanocomposite, the change of the reduced Young's modulus when the magnet is removed $\left(\Delta E^{*}=E_{B \neq 0}^{*}-E_{B=0}^{*}\right)$ is significant and about $15 \mathrm{kPa}$ (approx. $60 \%$ increase), $10 \%$ increase more in comparison to Chitosan single component constructs discussed previously (chapter 3, section 3.2).

\subsubsection{Oscillatory Shear Rheometry}

Figure 4.7 shows oscillatory shear rheometry results of experiments described in chapter 3. section 3.2 .2 .3 for the nanocomposite scaffolds fabricated with different $\mathrm{Fe}_{3} \mathrm{O}_{4}$ configurations.

In order to quantify the difference in $G^{\prime}$ and $G^{\prime \prime}$ given the presence and absence of the field, the difference of the mean values of the three measurements in each polymer, at 1 Hz were calculated as $\Delta G=G_{B \neq 0}-G_{B=0}$ and summarised in table 4.1 .

\begin{tabular}{|c|ccccc|}
\hline$(\mathrm{kPa})$ & Horizontal & $45^{\circ}$ & Not-oriented & Chitosan only & PDMS \\
\hline$\Delta \mathrm{G}^{\prime}$ & 4.3 & -2.11 & 7.2 & 1.5 & no significant \\
$\Delta \mathrm{G}^{\prime \prime}$ & 1.38 & no significant & -1.7 & -0.5 & no significant \\
\hline
\end{tabular}

Table 4.1: Average difference of the loss $G^{\prime \prime}$ and storage modulus $G^{\prime}$ when the magnet is removed $\left(\Delta G=G_{B \neq 0}-G_{B=0}\right)$ measured at $1 \mathrm{~Hz}$ for all nanocomposites, Chitosan single component and PDMS controls.

Table 4.1 shows the overall behaviours of all of the fabricated materials. A higher moduli is observed in the absence of the field for the horizontal construct for both $G^{\prime}$ and $G^{\prime \prime}$ as well as for $G^{\prime}$ chitosan single component and not oriented constructs. On the other hand, the moduli of the material when the magnet is removed is smaller for $G^{\prime \prime}$ 
a)

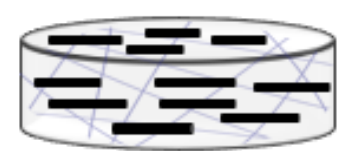

Horizontal

b)

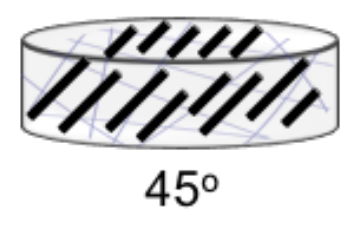

c)

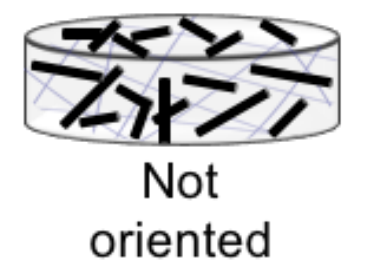

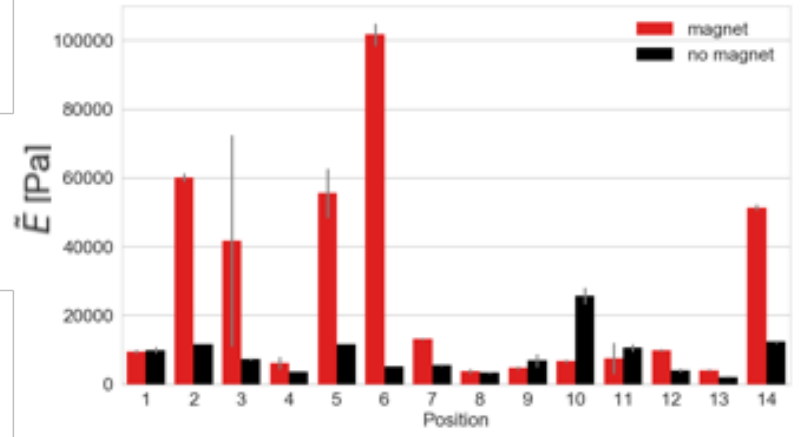
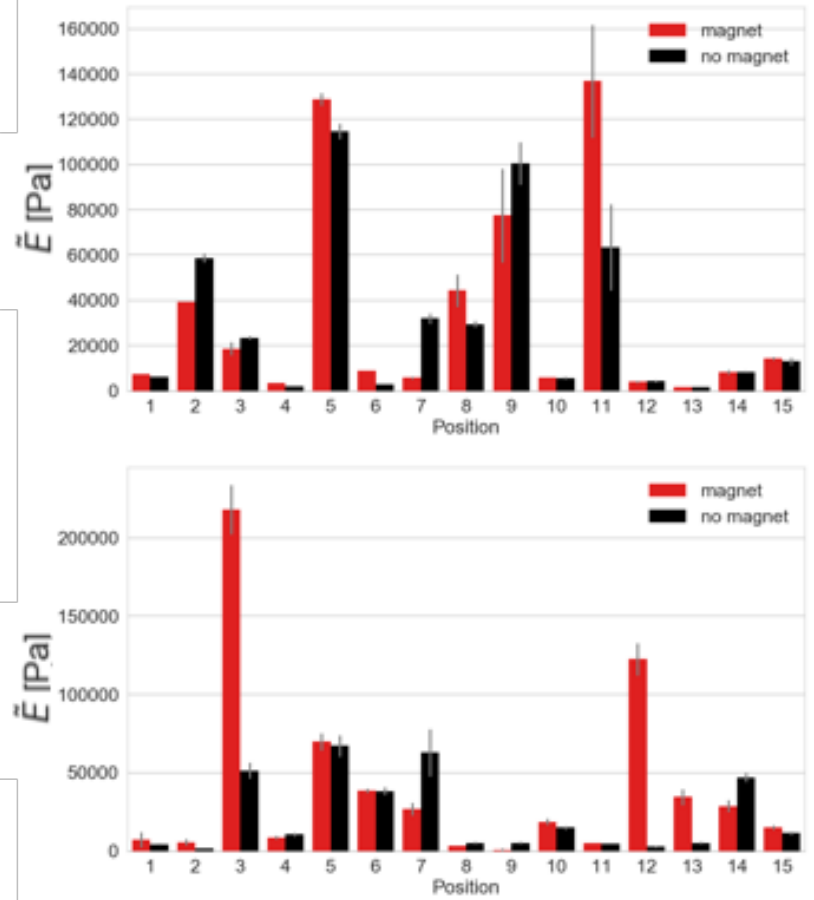

d)

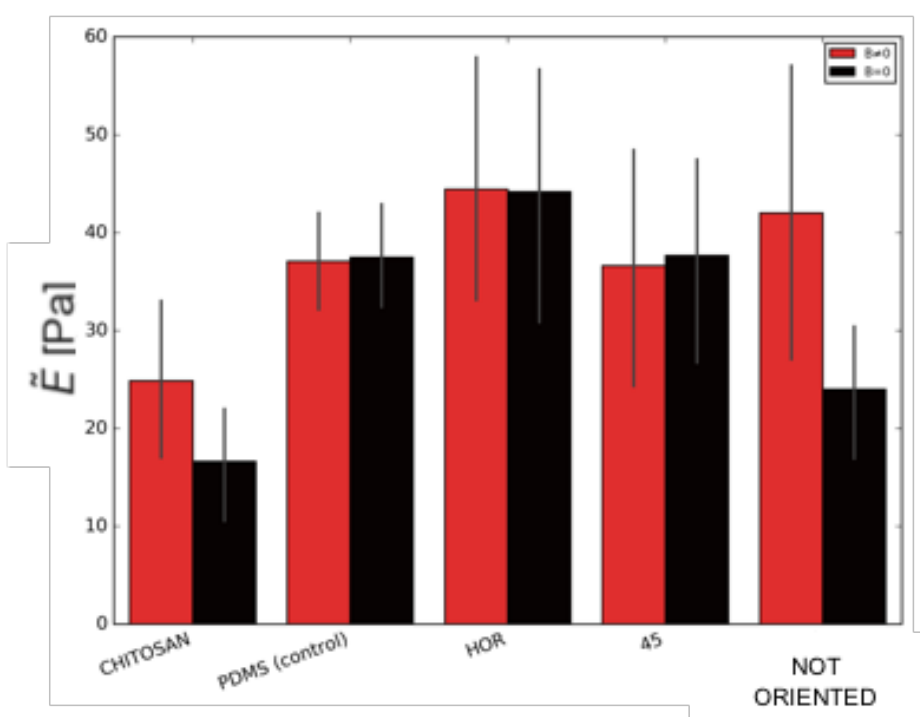

Figure 4.6: Chitosan nanocomposite polymers AFM microindentations. (a), (b) and (c) show the mean $\tilde{E}$ value of 100 curves recorded at each position in horizontal, $45^{\circ}$ and not-oriented polymers with a schematic representation of the fabricated materials. (d) Mean $\tilde{E}$ of Chitosan single component, PDMS control, horizontal, $45^{\circ}$ and notoriented configurations comprising all of the indented points. 
$\mathrm{G}^{\prime}$

a)

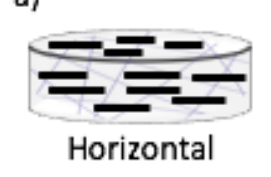

b)

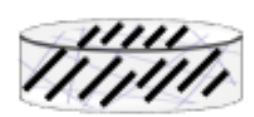

$45^{\circ}$

c)

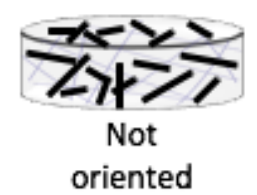

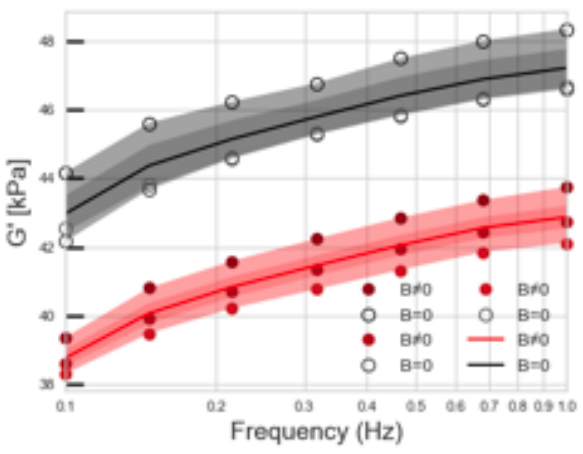
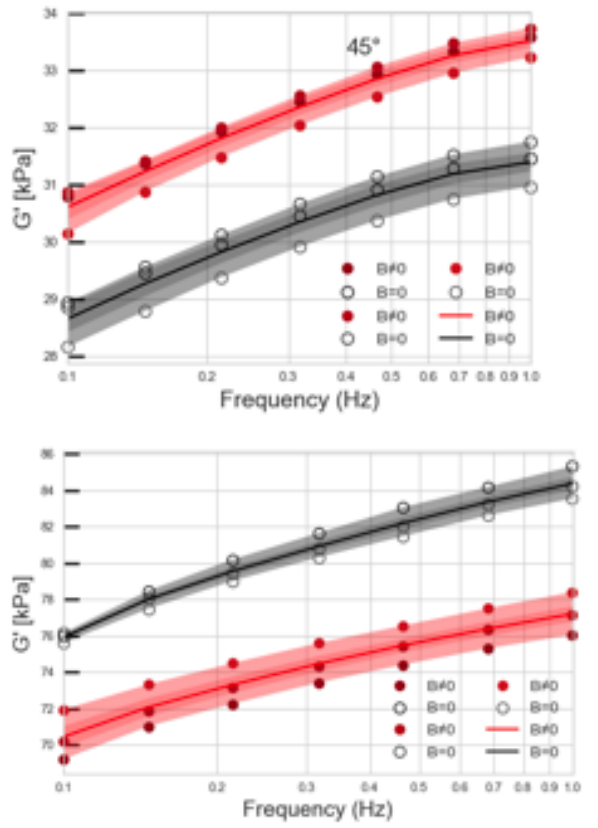

$\mathrm{G}^{\prime \prime}$
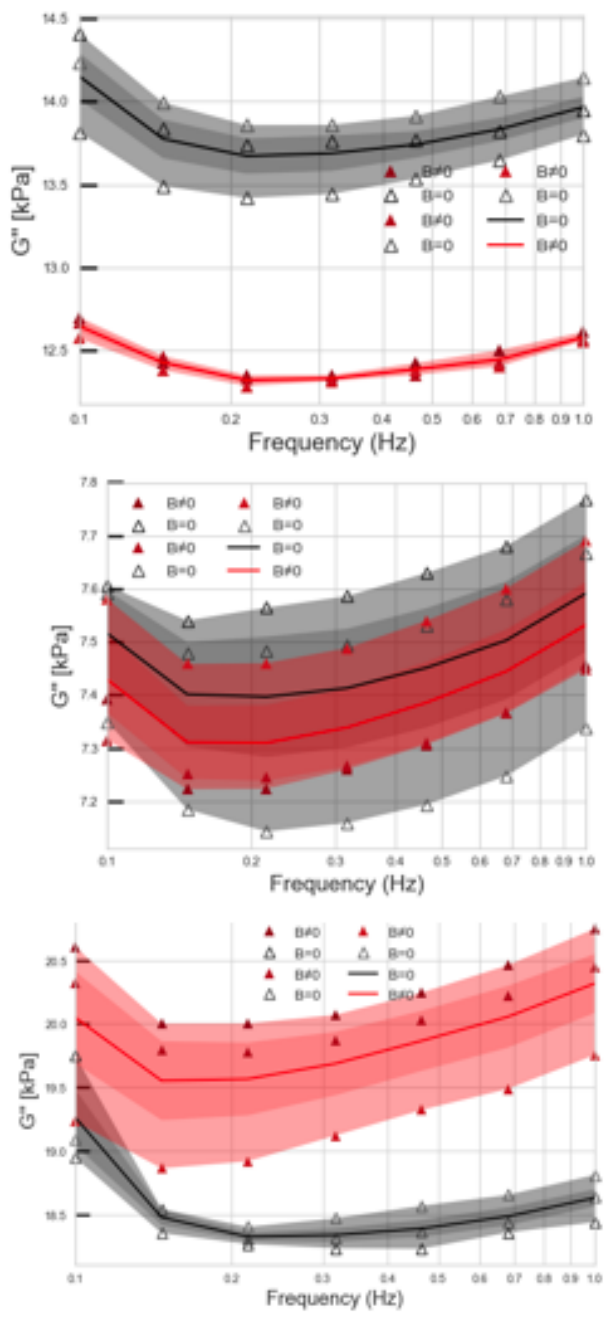

Figure 4.7: Bulk oscillatory shear rheometry experiments on chitosan nanocomposites. Semilog plot of $G^{\prime}$ and $G^{\prime \prime}$ obtained for (a) horizontal, (b) $45^{\circ}$ and (c) notoriented nanocomposite configurations. The black and red markers correspond to repetitions of frequency sweeps done in the absence and in presence of the $\vec{B}$, respectively. The black and the red lines represent the averages of the different repetitions. The shaded areas are confidence intervals of 68,95 and $99.7 \%$. 
not-oriented, chitosan single component constructs and $G^{\prime} 45^{\circ}$ construct.

Interestingly, Chitosan single component and the not-oriented nanocomposite scaffolds have similar behaviours. For both polymers, the loss modulus gets higher and the storage

modulus lower when the $\vec{B}$ is present. Yet, $G^{\prime}$ of the not-oriented composite is almost five times lower and $G^{\prime \prime}$ at least three times higher than Chitosan single component constructs.

\subsection{Discussion and conclusions}

In this chapter we have described the synthesis of $\mathrm{Fe}_{3} \mathrm{O}_{4}$ magnetic nanowires by a hydrolysis reaction. These nanowires were structurally characterised via AFM, SEM and TEM as well as in their composition via EDX which confirmed the presence of Fe and $\mathrm{O}$ in the crystalline structure. Future magnetic characterisation is suggested since the used reaction has reported superparamagnetism of the nanowires [267].

This nanowires were then used in the fabrication of nanocomposite hydrogels composed of a chitosan polymeric mesh with $\mathrm{Fe}_{3} \mathrm{O}_{4}$ nanowire clusters inside the polymer. These were fabricated in the presence of a $\vec{B}$ in different orientations (horizontal, $45^{\circ}$ ) to give the nanowires a certain degree of orientation and no $\vec{B}$ (not-oriented). SEM morphological characterisation (shown in figure 4.5) of horizontal (e) and $45^{\circ}$ (c) configurations show clusters of nanowires aligned in the surface of the chitosan.

Furthermore, chitosan single component and the other nanocomposite scaffolds show a difference in pore size and morphology maybe due to the presence and interactions of the polymer with the $\mathrm{Fe}_{3} \mathrm{O}_{4}$ nanowires, since chitosan is known to make complexes with $\mathrm{Fe}$ [268].

Local and bulk mechanical characterisation of all the nanocomposites was done by AFM microindentations and shear rheometry, respectively.

The maximum magnetic moment of the nanowires inside the scaffold is dependent on 
the viscosity and crosslinking of the gel.

Local AFM mechanical characterisation shows an overall not significant change for oriented matrices but a significant change of the reduced modulus of the not-oriented nanocomposite of about $15 \mathrm{kPa}$ (approx. 60\% increase), $10 \%$ increase more in comparison to chitosan single component constructs when the $\vec{B}$ is removed $\left(\Delta E^{*}=E_{B \neq 0}^{*}-E_{B=0}^{*}\right)$. Furthermore, we proof the mechanical strengthening of the reinforced chitosan scaffolds by embedding nanowires in the polymeric lattice.

Bulk rheometry mechanical characterisation shows similar behaviours between the notoriented nanocomposite and chitosan single component scaffolds. Not-oriented nanocomposite shows an enhanced behaviour of 3 times higher loss modulus observed for chitosan single component and the storage modulus getting 5 times lower in the presence of the $\vec{B}$.

As described before in chapter 3, chitosan's mechano-magnetic coupling could be explained by magnetoydrodynamic effects and the material polarisation by a mechanical deformation in the presence of a $\vec{B}$. If an electrically conductive medium flows through the porous hydrogel, it creates a Lorentz force due to an external $\vec{B}$ which, in turn, affects the mechanical properties of the piezoelectric hydrogel.

It is interesting to note that shear rheometry shows that for the not-oriented nanocomposite and chitosan single component hydrogels, the presence of the $\vec{B}$ results in an increase of the viscous behaviour (loss modulus) and decrease of the elastic behaviour (storage modulus). This behaviour maybe due to the polarisation of the material and a magnetohydrodynamic effect as discussed in the previous chapter. Furthermore, the presence of the $\vec{B}$ increases the Young's modulus of the material (measured by AFM) probably due to tensing the polymeric mesh. These behaviours measured by these two experimental approaches cannot be directly compared, despite that they confirm a magneto-mechanical coupling in the materials. AFM microindentions measure the Young's Modulus or tensile elasticity of the material via a normal deformation whereas shear oscillatory rheometry measures the 
oscillatory shear stress and determines the material's elastic and viscous responses.

In order to clarify the observed behaviour, future experiments could include AFM mapping of storage and loss modulus via dynamic microindentations which would rely on applying an oscillatory normal force at different frequencies to assess time dependence of the moduli in the micron scale, as well as axial DMA (dynamic mechanical analysis) to study the viscoelastic macro-scale behaviour applying normal forces.

In the case of these nanocomposite materials, the enhanced magneto-mechanical coupling observed might result in magnetostriction (change of dimensions of a material due to the presence of a $\vec{B}$ ) of the hydrogel. This was observed during AFM microindentation experiments by the movement of the piezo when the cantilever was in contact with the sample and a change in $\vec{B}$ was applied. A higher intensity of the applied $\vec{B}$ is necessary for any future experiments in order to quantify this behaviour.

Drawbacks of these materials are inhomogeneities in the measured mechanical properties intrinsic of porous materials and enhanced due to the presence of not perfectly dispersed nanostructures within the polymer. These problems can be addressed by the use of stronger and more homogeneous magnetic fields to align the nanowires further and avoid the formation of clusters.

Our results show a greater mechano-magnetic coupling behaviour of nanocomposite constructs in comparison to chitosan single component scaffolds. Magnetically controlled mechanical properties of biomaterials used in TE could offer the possibility to direct cellular differentiation on demand by changing the desired stiffness of the scaffold given a magnetic field $(\vec{B})$. Additionally, magnetically actuating the material could improve the diffusion of nutrients, drugs and growth factors. 


\section{Chapter 5}

\section{High-speed rotating microparticles}

The work presented in this chapter is being prepared for submission to the 'ACS Applied Materials and Interfaces' Journal for publication.

The synthesis protocols of the microellipsoids have been patented in the United Kingdom (No. 1616191.1) and an international application (application No. PCT/GB2017/052852) has been filled in September 2017.

Polymeric magnetic spherical microparticles are employed as sensors/actuators in lab-ona-chip applications, in small-scale robotics and in biomedical assays. Much of their future potential depends on the precise control of their motion and stability with low magnetic fields; this requires magnetic anisotropy, which is difficult to achieve in spheres.

In this chapter, I describe a simple method to synthesise anisotropic ellipsoidal particles

by applying a $\vec{B}$ during synthesis, using a composite of polycaprolactone (PCL) and $\mathrm{Fe}_{3} \mathrm{O}_{4}$ nanowires (average eccentricity of $0.60 \pm 0.14$ ). The particles are thoroughly characterised using optical microscopy and SEM. Their suitability for magnetic control is demonstrated by analysing their rotation in low magnetic fields $(0.1,1,5,10$ and $20 \mathrm{mT})$ at varying 
rotational frequencies $(1 \mathrm{~Hz}$ and $5 \mathrm{~Hz})$.

The microellipsoids clearly outperform spherical commercial microparticles at following rotating fields. Furthermore, only $23 \%$ of commercial particles analysed rotated at $1 \mathrm{~Hz}$ and $26 \%$ at $5 \mathrm{~Hz}$; whereas $77 \%$ of our ellipsoidal particles rotated at $1 \mathrm{~Hz}$, and $74 \%$ did at $5 \mathrm{~Hz}$.

\subsection{Introduction}

Magnetic polymer composites (MPCs) are widely used in microsystems and microrobotics as they simultaneously feature the processability and facile chemical functionalisation of polymers, and the possibility of using magnetic fields for actuation by exploiting the magnetic properties of the embedded nanostructures 227,269271 .

Magnetic fields are ideal external force generators due to their non-invasiveness, biological inertness, and better performance in comparison to other methods such as acoustic waves (including ultrasound) and electric fields [100]. MPC-based microstructures can be used to perform tasks in handling and assembling small objects and biological molecules, or sensing physical, biological or chemical molecules or functions $269,270,272,273$. They are also used for generating and measuring forces at the micrometre scale in biophysical studies 28, 145, 274], for microrheology of complex fluids and for magnetic tweezer studies 146,147 .

Magnetic spherical beads have been preferred as a favourite architecture for small-scale mechanical technologies using soft materials due to the ease of fabrication, versatility and suitability for theoretical modelling.

Magnetic beads already play an important role in microfluidics; they can be made to link to target species and can be used for the manipulation and/or detection in labon-a-chip systems $158,275,276$ and protein and biomolecular purification and mixing. 
Magnetic microparticles have been used to study the bacterial flagellar motor in torquegenerating assays [155], cellular mechanotransduction and microrheology with magnetic twisting cytometry 277, 278], cellular, protein and nucleic acid manipulation in separation assays as immunomagnetic isolation [274], immunoassays [279], magnetic flow cytometry [280] or in magnetic separation in lab-on-a-chip microfluidic systems [281] and other microfabricated devices, as well as in directed hyperthermia applications [160, 161 and targeted drug delivery [159]. Most applications use commercial spherical MPC microparticles or colloids consisting of a polymeric matrix that confines magnetic spherical nanoparticles (NPs), mainly superparamagnetic $\mathrm{Fe}_{3} \mathrm{O}_{4}$ NPs.

One of the main drawbacks of using spherical microparticles confining NPs is that they have very little shape anisotropy. Relatively high magnetic fields are needed to align all of the magnetic moments of the NPs inside of them [161]. To obtain a more controlled magnetic response several strategies aimed at programming magnetic anisotropy in the structure have been developed $[270,282]$. Often these have involved top-down approaches such as inkjet printing [270 or photopatterning [282].

In this thesis, we describe the synthesis of magnetic nanocomposite microellipsoids based on a matrix of PCL by a simple oil-in-water emulsion method. The polymer microellipsoids are formed in the emulsion in the presence of magnetite nanowires $\left(\mathrm{Fe}_{3} \mathrm{O}_{4}\right)$, which are trapped in the particle matrix. During the synthesis, DC magnetic fields are used to align the nanowires and create magnetic anisotropy. Interestingly, the interplay between nanowire-polymer interactions, and the torques generated on the nanowires by applying external magnetic fields during microellipsoid synthesis and hardening, results in the formation of magnetic clusters with nanowires aligned in the same direction, which in turn cause the particles to become ellipsoidal. The particles are characterised using optical microscopy and SEM. Furthermore, the superior rotation and torque by rotating magnetic fields at low intensity in comparison to commercially available microparticles is 
demonstrated using rotating magnetic fields with field strengths of $0.1,1,5,10$ and 20 $\mathrm{mT}$, at varying rotation frequencies $(1 \mathrm{~Hz}$ and $5 \mathrm{~Hz})$. At higher magnetic fields, the performance of the ellipsoidal particles is superior or equal to that of the commercial spherical particles. At the low $\vec{B}$ strengths $(0.1 \mathrm{mT}$ and $1 \mathrm{mT})$ that are required for most practical applications, our particles clearly outperform the commercial ones.

\subsubsection{Magnetic shape anisotropy in colloidal composites}

Composite spherical superparamagnetic microparticles with embedded NPs have been the preferred commercial strategy to prepare magnetic actuators at the micron scale. This approach results in the need of high magnetic fields given their little shape anisotropy as shown in figure 5.1 a). In the absence of a $\vec{B}(\vec{B}=0)$, the magnetic moment of each one of the embedded NPs rotates freely in all directions called the Neel relaxation. In the presence of a field $(H \neq 0)$, the moments of the NPs fluctuate around the direction of the external field and when the applied field is strong enough, the magnetic moments of the NPs align completely to the direction of the applied external field [161]. The magnetic response of the colloid can be represented with a magnetic dipole aligned to the external field (depicted as the blue arrow on top of the microparticle).

Figure 5.1 b) shows a schematic representation of the microellipsoidal composites with nanowires semi-aligned into clusters by the application of a $\vec{B}$ during synthesis, resulting on a ellipsoidal shape, hence higher magnetic shape anisotropy which results on a faster alignment of the ellipsoid to the longitudinal easy axis of magnetisation, in comparison to the spherical commercial counterparts. 
a)

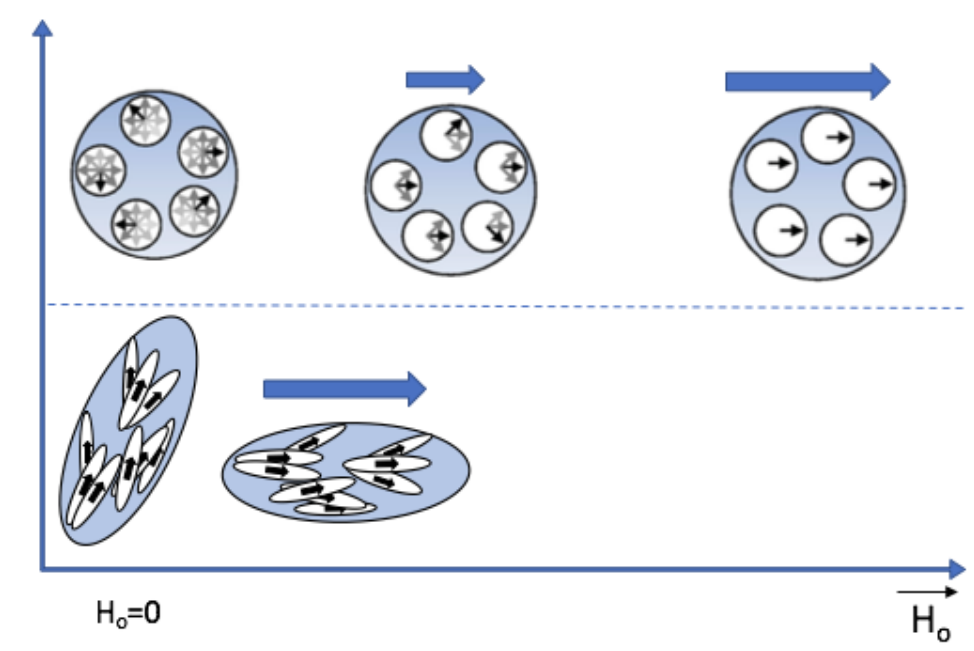

Figure 5.1: Schematic representation of the magnetic response of ellipsoidal colloids that correspond to the magnetic dipole aligned to the external field (shown as a blue arrow on top). (a) Spherical polymeric colloids with embedded NPs. The intensity of the magnetic response increases with the intensity of the applied field and is zero in the absence of external field. (Modified from [161]) (b) Ellipsoidal polymeric colloids with clusterized nanowires. The magnetic response is stronger at smaller applied fields given the ellipsoidal colloidal shape.

\subsection{Materials and methods}

\subsubsection{Synthesis of ellipsoidal microparticles}

Microellipsoids were fabricated by using the oil-in-water emulsion method as depicted in 5.2 Fig. 1. The oil phase comprised a dilution of $0.5 \mathrm{mM}$ PCL (average molecular weight: 80,000) and $2.5 \% \mathrm{w} / \mathrm{v}$ Luperox (benzoyl peroxide), which acted as the initiator of PCL crosslinking, both dissolved in dichloromethane.

Synthesised $\mathrm{Fe}_{3} \mathrm{O}_{4}$ nanowires (described above) were dispersed in the PCL solution at a concentration of $0.5 \%(\mathrm{w} / \mathrm{v})$ which is at least 20 times smaller concentration than commercial particles ( $\approx 10$ to $39 \%$ of $\mathrm{Fe}_{3} \mathrm{O}_{4}$ content depending on the manufacturer). In order to achieve a homogeneous dispersion of nanowires in the oil phase, the solution was sonicated in a water bath for 5 minutes. 
The water phase consisted of soluble poly(vinyl alcohol) (PVA) $(1.5 \% \mathrm{w} / \mathrm{v})$ as a nonsurfactant stabiliser. The oil phase was emulsified by adding it dropwise into the water phase while stirring at 3,000rpm (MS1 Minishaker IKA), and emulsified for 10 minutes at this speed. Liquid agar $(1 \% \mathrm{w} / \mathrm{v})$ was immediately added and mixed for 3 minutes. The gel was settled in the freezer at $-20^{\circ} \mathrm{C}$ for 10 minutes to immobilise the microellipsoids during

hardening. A static $\vec{B}$ of $0.25 \mathrm{~T}$ was applied during hardening to modify the orientation of the nanowires embedded within the polymeric structure. The gel was aged at room temperature overnight to allow particle hardening. Finally, microellipsoids were cleaned and sorted by size by means of density gradient centrifugation with a glucose column at 3,000 rpm for 7 minutes. The microellipsoids were cleaned 3 times with ultrapure deionized (DI) water (Millipore MilliQ) and kept at $4^{\circ} \mathrm{C}$.

\subsubsection{Microparticle morphology and structural characterisation}

The microstructure and phase analyses of the microparticles and nanowires were performed by TEM on a FEI Talos F200X as described in chapter 4 , section 4.2.3.

SEM were acquired by a secondary electron detector in Zeiss ULTRA 55. The sample was dispersed on a Si wafer and dried.

\subsubsection{Microparticle rotational experiments}

The rotational motion of microparticles was characterised using a commercial magnetic actuation system (MFG-100-i, Magnebotix, Switzerland) mounted on optical microscope (Olympus IX, Olympus). The magnetic actuation system consists of 4 pairs of solenoid coils, which allow 3D manipulation of small-scale magnetic objects. Experiments were carried out in ultrapure DI water deionized water (Millipore MilliQ) applying different rotating magnetic fields $(0.1,1,5,10$ and $20 \mathrm{mT})$ at different frequencies (1 and $5 \mathrm{~Hz})$. Commercial 


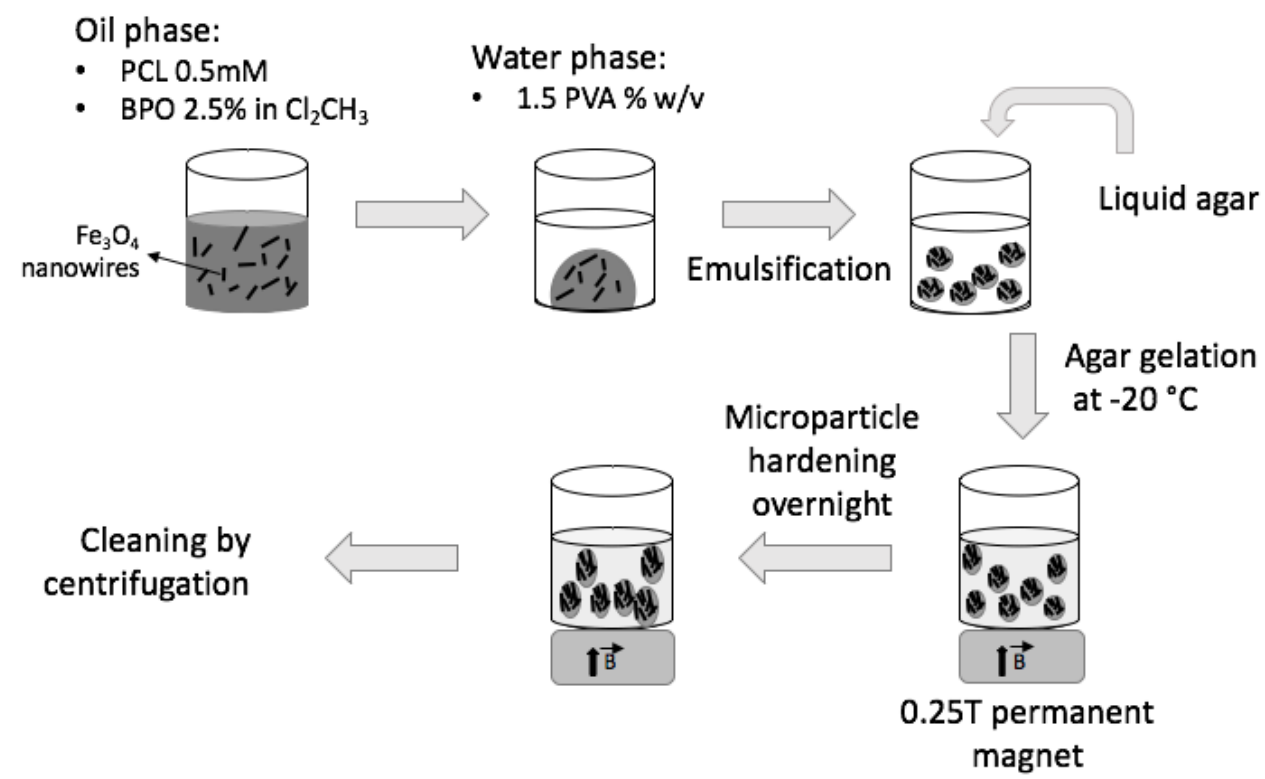

Figure 5.2: Schematic representation of the oil-in-water emulsion fabrication of the microellipsoids. The oil phase contains benzoyl peroxide $(2.5 \% \mathrm{w} / \mathrm{v})$ and $0.5 \mathrm{mM}$ PCL dissolved in dichloromethane with $0.5 \% \mathrm{Fe}_{3} \mathrm{O}_{4}$ nanowires dispersed. The water phase consists of PVA $1.5 \% \mathrm{w} / \mathrm{v}$. The oil phase was emulsified into the water phase by stirring. Liquid agar $(1 \% \mathrm{w} / \mathrm{v})$ was immediately added and settled at $-20^{\circ} \mathrm{C}$ during microellipsoid hardening in the presence of a static $\vec{B}$ of $0.25 \mathrm{~T}$. Once agar is not liquid, particles were left at room temperature overnight to allow PCL hardening while inducing orientation of the nanowires and creating the ellipsoidal shape. 
superparamagnetic spherical beads (SPHERO Magnetic Particles: PM-20) were assessed as a control experiment. Particle motion was traced from the image sequences, recorded using charge coupled device (SCA 1400, Basler) at 30 frames per second (fps).

\subsubsection{Analysis of rotational experiments}

The image processing to analyse the rotation of the microellipsoids was performed by Dr. Isabel Llorente at University College London. A suite of image processing algorithms for single particle rotational tracking were custom-written in Matlab by Dr. Llorente to quantitatively extract the orientation of all particles in each acquired video sequence.

The analysis calculates the major $a$ and minor $b$ axis of each particle and links the particles found on subsequent frames by comparing the pair-wise distances between each particle.

From these calculations, the number of rotations per second for all of the particles that gave a $360^{\circ}$ turn for 0.1 and $1 \mathrm{mT}$ and 1 and $5 \mathrm{~Hz}$. These rotations were calculated by counting the number of turns of the long axis a and dividing by the number of seconds in a video.

Furthermore, taking in account the moment when each particle (microellipsoid and microparticle) was rotating at a similar frequency than the applied one, the angular velocity was calculated and the exerted torque was approximated (results for torque calculations are shown in appendix B .

\subsection{Results and discussion}

\subsubsection{Morphology and structural characterisation}

Ellipsoidal microparticles were fabricated as described in section 5.2.1. Figure 5.3 (a) shows two typical microellipsoids imaged by SEM showing clear ellipsoidal shape with different 
eccentricities $(\varepsilon)$. The two particles present a different eccentricity $\varepsilon$; that represents how ellipsoidal $(\varepsilon=1)$ or spherical $(\varepsilon=0)$ a particle is, it can be calculated using the following formula:

$$
\varepsilon=\sqrt{\frac{a^{2}-b^{2}}{a^{2}}}, 0<\varepsilon<1
$$

for prolate ellipsoids with semiaxis $a, b$.

PCL microparticles fabricated without nanowires and in the absence of a $\vec{B}$ using this method present a spherical shape, as shown in figure 5.4 .

TEM was used to identify the arrangement of the $\mathrm{Fe}_{3} \mathrm{O}_{4}$ nanowires within the particle (Fig. 5.3 b). The lower panel of Fig 5.3 (b) shows clusters of nanowires. The presence of a $\vec{B}$ during synthesis, results in the formation of clusters (shown in (c)) which eventually determines the final ellipsoidal shape of the particle. It is possible that the $\vec{B}$ applied during the synthesis is not sufficient to overcome other forces arising from the interaction between polymers and nanowires during crosslinking and colloid hardening, preventing nanowires from aligning completely to the applied $\vec{B}$, but being enough to orient clusters of wires into an ellipsoidal shape.

The presence of the $\vec{B}$ unequivocally results in particle elongation, ellipsoidal shape and hence colloid magnetic shape anisotropy. Figure 5.4 shows colloids consisting of PCL fabricated by the same oil-in-water emulsion method as described in the microellipsoid synthesis section (oil phase consisting of Luperox $2.5 \% \mathrm{w} / \mathrm{v}$ and $0.5 \mathrm{mM}$ PCL dissolved in dichloromethane; water phase PVA $1.5 \% \mathrm{w} / \mathrm{v}$; no nanowires embedded). These particles show higher sphericity in comparison to the ellipsoidal microparticles described in the main text (with $0.5 \% \mathrm{w} / \mathrm{v}$ magnetic $\mathrm{Fe}_{3} \mathrm{O}_{4}$ nanowires embedded in the polymeric matrix and aligned in the presence of a $0.25 \mathrm{~T} \vec{B}$ during hardening).

In order to quantify $\varepsilon$ for the fabricated microellipsoids, the major and minor axis of 


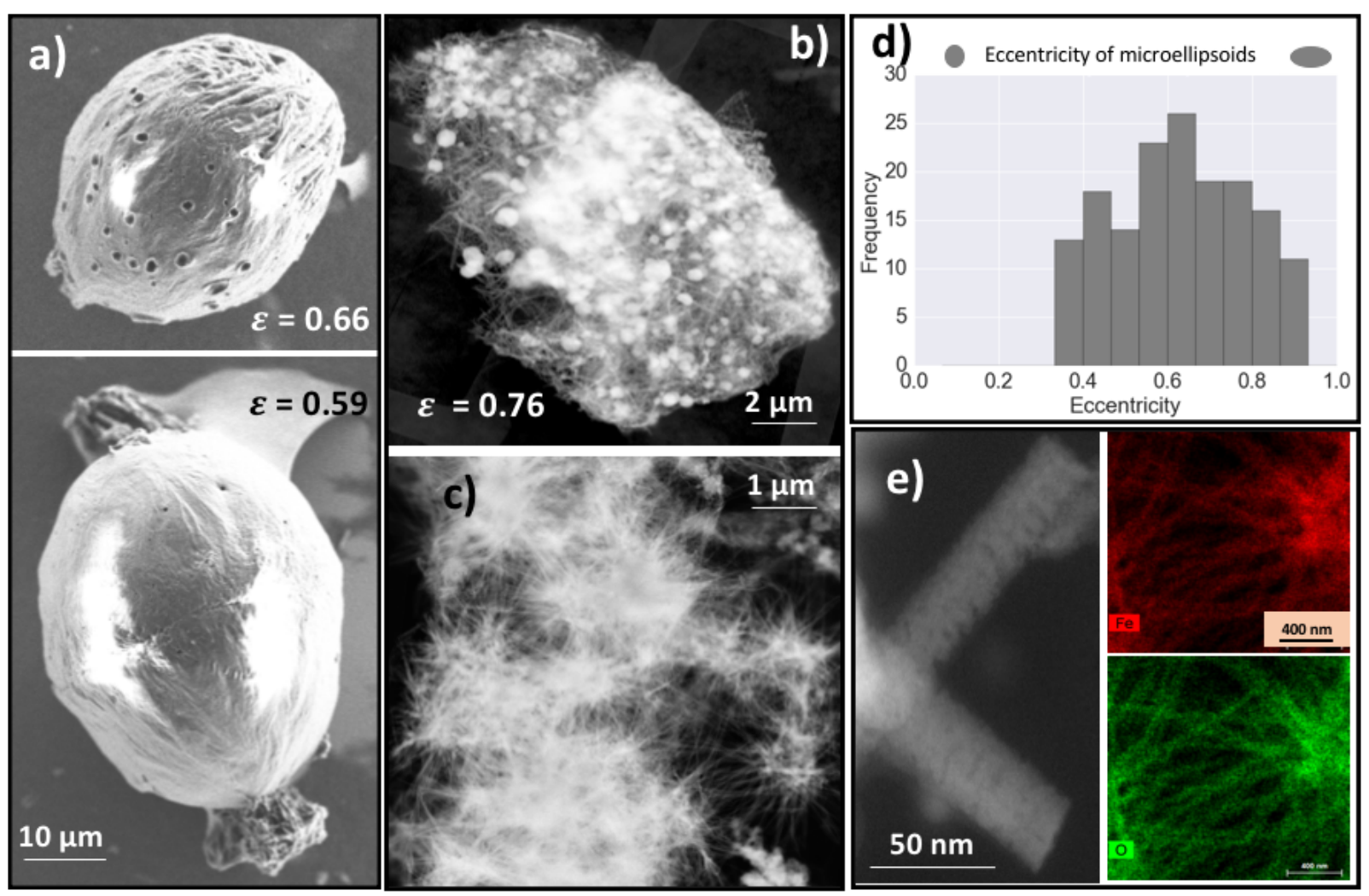

Figure 5.3: (a) SEM images of fabricated magnetic ellipsoidal particles with different eccentricities $\varepsilon$. (b) TEM images of microellipsoids showing the nanowire structure embedded within the polymer matrix. (c) Nanowire cluster formation due to the presence of a $\vec{B}$ during microparticle hardening. (d) Histogram of $\varepsilon$ values of the microellipsoids. (e) TEM image of fabricated $\mathrm{Fe}_{3} \mathrm{O}_{4}$ nanowires and EDX analysis showing the structural composition of the nanowires (see fig. 4.4)

the particles were measured as described above (see 5.2.3.1 section). The distribution of $\varepsilon$ is shown in figure 5.3 (d), for a total of 159 colloids analysed, showing that the synthesis method clearly leads to ellipsoidal particles.

Finally, figure 5.3 (e) shows a TEM analysis of individual nanowires. EDX analysis of the nanowires embedded within the polymeric PCL lattice is also shown in (e) where iron content is depicted in red and oxygen in green (see fig. 4.4 for details). 


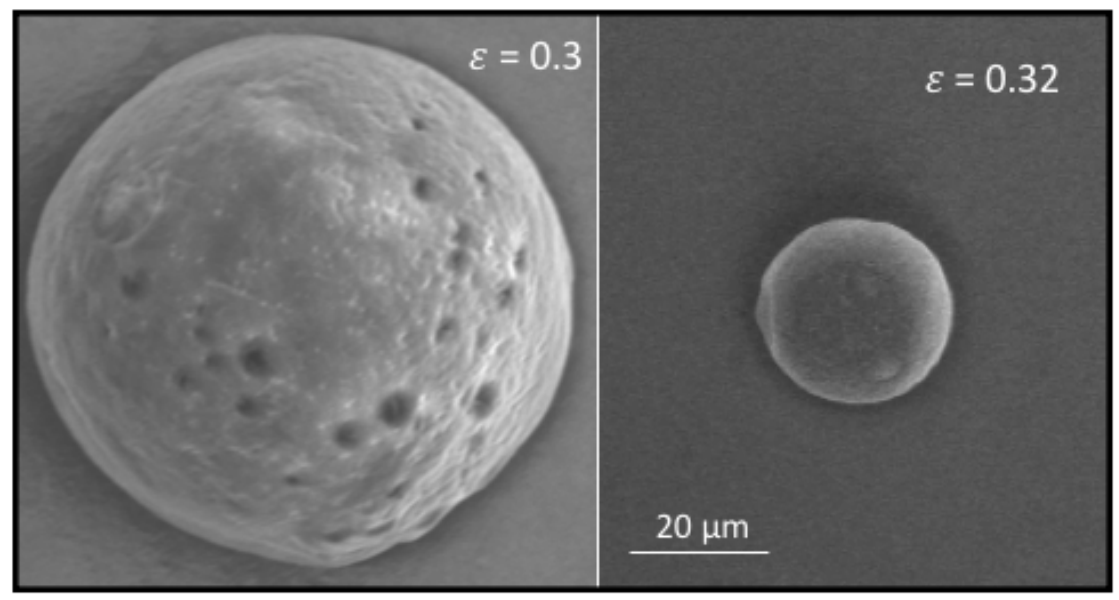

Figure 5.4: PCL only colloids

SEM images of PCL colloids without nanowires and without $\vec{B}$ applied during the synthesis showing higher sphericity.

\subsubsection{Rotations per second calculation}

Figure 5.5 (Dr. Isabel Llorente) (a) and (c) show a time sequence for the rotation of one of the microellipsoids in the presence of a $10 \mathrm{mT} \vec{B}$ rotating at a frequency of $1 \mathrm{~Hz}$ and $5 \mathrm{~Hz}$. The initial orientation angle obtained is between -90 degrees and +90 degrees, and particle rotation is indicated by the clear periodicity of the angle versus time (blue traces in Figs. 5.5 (b) and (d). Further processing of the angle is carried out by automatically detecting orientation jumps in the data and assuming anti-clockwise rotation to generate a monotonically increasing positive angle (red traces in Figs. 3b and 3d). This can be fitted to a line (solid black lines in Figs. 3b and 3d) to obtain from the slope the angular velocity and frequency of rotation of the microellipsoid. Some of the particles analysed stop intermittently during rotation (likely due to interactions with the substrate). In these cases, only the linear segments of actual periodic rotation are detected automatically in the angle-versus-time data and analysed to obtain the slope for each segment. An average rotation frequency is calculated as the mean of the frequencies corresponding to all rotation segments in these cases. Particle trajectories with less than 10 frames and rotation segments 
with less than 5 frames are excluded from the analysis. Only isolated particles are analysed (particles that clump together into large clusters are discarded).

Figure 5.6 shows violin plots of the number of rotations per second for each particle that gave at least one $360^{\circ}$ with an applied intensity of the field of $0.1 \mathrm{mT}$ and $1 \mathrm{mT}$ and 1 and $5 \mathrm{~Hz}$.

At the lowest intensity of the field, $0.1 \mathrm{mT}, 1 \mathrm{~Hz}$, the microellipsoids rotated at $0.35 \pm 0.19$ Hz. At $5 \mathrm{~Hz}$ applied field none of the microellipsoids rotated at more than $0.5 \mathrm{~Hz}$, yet, no commercial microparticle managed to give at least one $360^{\circ}$ turn.

In the case of $1 \mathrm{mT}, 1 \mathrm{~Hz}$, microellipsoids rotated in average $0.61 \pm 0.27 \mathrm{~Hz}$ with the best one rotating at $0.96 \mathrm{~Hz}$. With a $5 \mathrm{~Hz}$ external field, the maximum frequency of microellipsoids was $4.79 \mathrm{~Hz}$ compared to $2.57 \mathrm{~Hz}$ of the commercial particles. These results show that the synthesised ellipsoidal colloids outperformed the spherical commercial microparticles.

A comparison of microparticles and microellipsoids subject to a $1 \mathrm{mT}, 1 \mathrm{~Hz} \vec{B}$ is shown in figure 5.7. (a) shows the orientation angle vs. time graph for the particles that rotated closest to the applied frequency for each case (fabricated microellipsoid and commercial). The number of rotations per second are shown at the bottom of each colloid's graph. (b) Shows a comparison of the angle before post-processing for the same particles. A clear oscillation of the commercial microparticle is observed and circled in black. Several oscillations repeat throughout the 5 second video, lowering the rotations per second to $0.48 \mathrm{~Hz}$ in comparison to $0.94 \mathrm{~Hz}$ of the fabricated microellipsoid.

\subsubsection{Angular velocity calculation}

Using the method described above, the rotation of the microellipsoids in the presence of a rotating $\vec{B}$ of different intensities $(0.1,1,5,10$ and $20 \mathrm{mT})$ and varying the rotational frequency $(1 \mathrm{~Hz}$ and $5 \mathrm{~Hz})$ was measured by analysing optical microscopy videos of the particles as they were being actuated by a rotating $\vec{B}$, following the procedure described 
a)

polymeric magnetic micro-ellipsoid rotating in $10 \mathrm{mT}, 1 \mathrm{~Hz}$ magnetic field

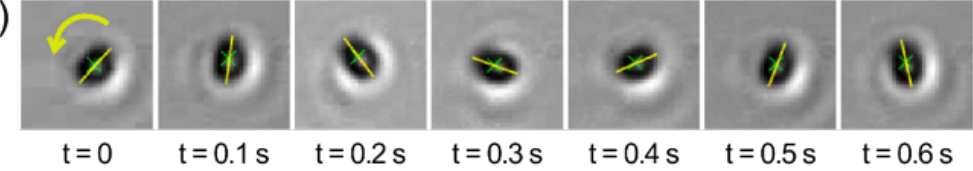

b)

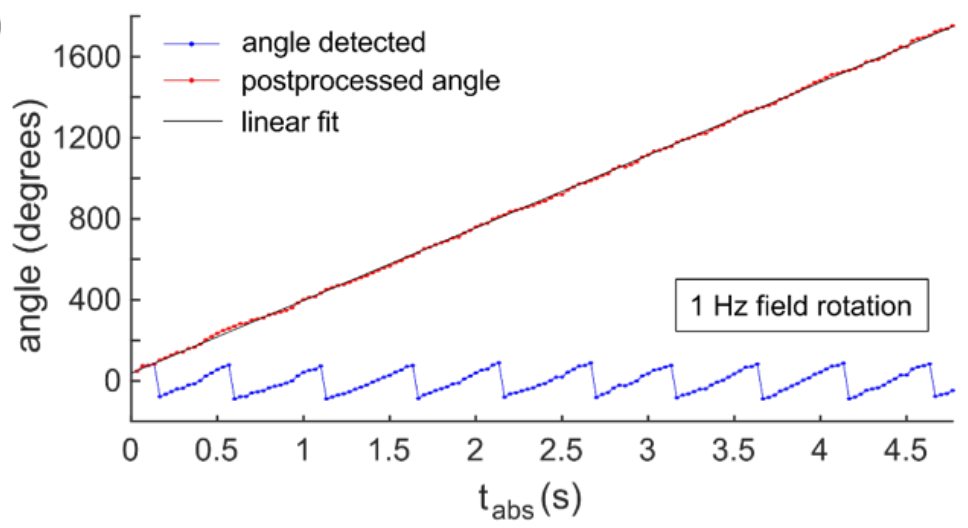

c)

polymeric magnetic micro-ellipsoid rotating in $10 \mathrm{mT}, 5 \mathrm{~Hz}$ magnetic field

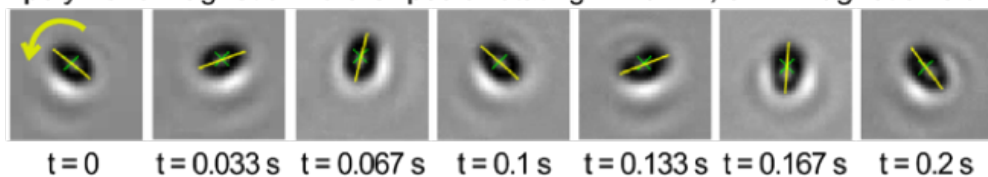

d)

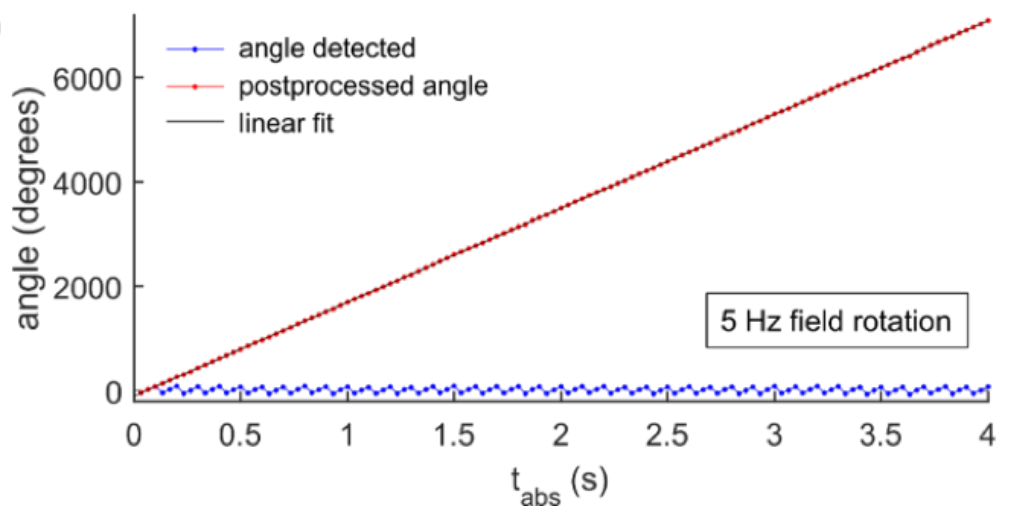

Figure 5.5: Quantitative extraction of particle rotation angle and frequency via image processing. (a) Sequence of image frames acquired for one of our polymeric magnetic microellipsoids rotating in a $10 \mathrm{mT}, 1 \mathrm{~Hz}$ rotating $\vec{B}$. Every third frame acquired is shown. Yellow lines indicate the angle found for the particle. Green crosses indicate the found centre-of-mass. The rotation of the field and particle is counterclockwise as indicated by the rotation arrow on the first frame. (b) Initial orientation angle versus time (blue line) for a $\sim 5$-second-long rotation sequence corresponding to the particle shown in a). The fit of the post-processed angle (red line, see text) yields a particle rotation frequency of $(0.996 \pm 0.001) \mathrm{Hz}$, very close to the field-rotation frequency. (c) Rotation time sequence for one of our particles in the presence of a 10 $\mathrm{mT}, 5 \mathrm{~Hz}$ rotating $\vec{B}$. Every frame acquired is shown. (d) Orientation versus time for the particle rotating in the $5 \mathrm{~Hz}$ field. The linear fit (black line on red post-processed angle) yields a particle rotation frequency of $(4.995 \pm 0.001) \mathrm{Hz}$ (Dr. Isabel Llorente) 


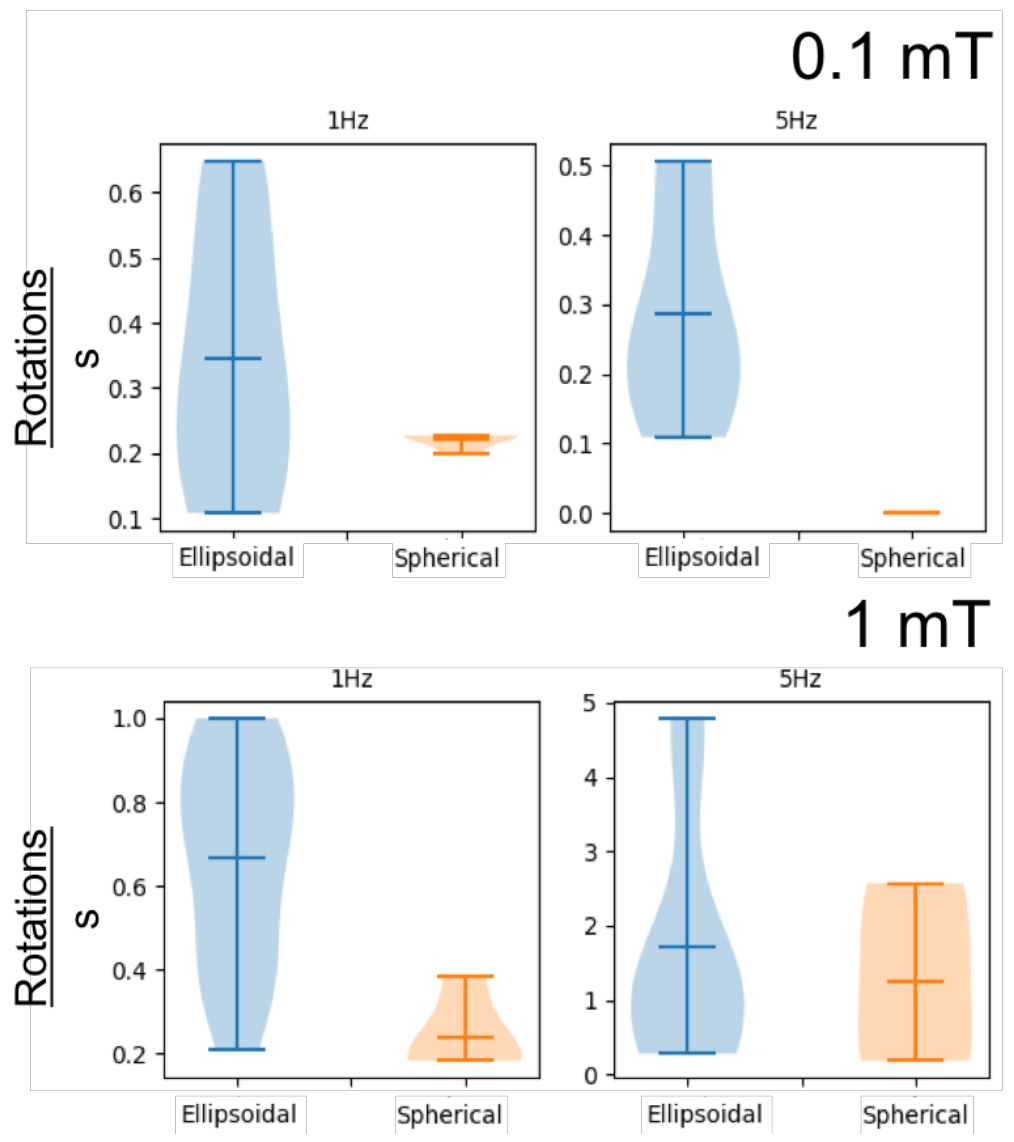

Figure 5.6: Violin-plots of rotations per second of microparticles (red) and microellipsoids (blue) in the presence of a $\vec{B}=0.1 \mathrm{mT}$ (top) and $1 \mathrm{mT}$ (bottom) with an angular velocity of 1 and $5 \mathrm{~Hz}$. 
$1 \mathrm{mT}, 1 \mathrm{~Hz}$

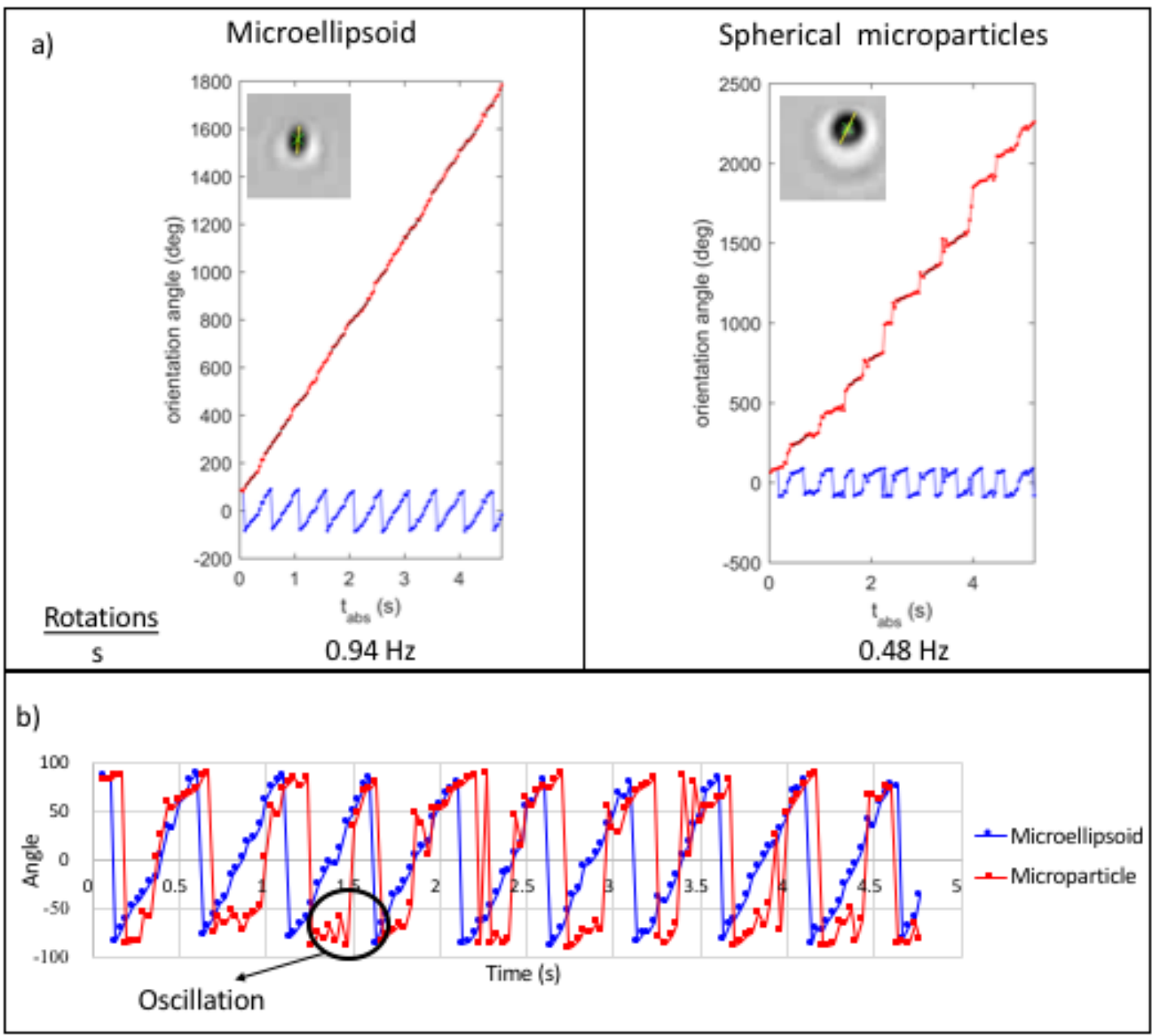

Figure 5.7: Comparison of spherical (commercial) microparticles and microellipsoids rotating at $1 \mathrm{~Hz}$ with a $\vec{B}$ of $1 \mathrm{mT}$. (a) Orientation angle vs. time of ellipsoidal (left) and spherical (right) colloids showing examples of rotating colloids. Numbers at the bottom are the observed rotations per second. (b) Comparison of orientation angle of the best ellipsoidal (blue) and spherical (red) colloids also depicted in a). An example of oscillations from spherical microparticles is circled in black. No oscillations are observed for the ellipsoidal colloids. 
in the materials and methods section and above.

Figure 5.9 shows the comparison of the angular velocity calculated for microellipsoids and the commercial spherical microparticles at $1 \mathrm{~Hz}$ (a) and $5 \mathrm{~Hz}$ (b) respectively when the particles were rotating at a frequency close to the applied one. Oscillations were not taken in consideration. Higher magnetic fields result in the particles' angular velocity to follow and match the angular velocity of the applied $\vec{B}$ for both kinds of particles because of a higher magnetisation resulting from more magnetic moments aligning in the direction of the $\vec{B}$, due to a stronger magnetic field. Even though, in all cases the ellipsoidal particles show an equal (at $20 \mathrm{mT}$ ) or better performance.

This better performance could be due to a combination of the intrinsic shape anisotropy of microellipsoids, along with the fact that these fabricated colloids might be lighter since they contain 20 times less concentration of $\mathrm{Fe}_{3} \mathrm{O}_{4}$. Because of this and differences in shape, spherical colloids might be subject to higher friction than ellipsoidal ones, making them lag behind and rotate slightly slower than the applied field.

A total of 400 particles were analysed for the results presented in Fig. 5.9. Although over a hundred particles were analysed for each condition, only data for a few particles was useful for quantitative analysis, since only particles able to perform at least a complete $360^{\circ}$ turn were considered. The graphs indicate the fraction of particles that fulfil this criterion. It is important to note that a larger number of ellipsoidal particles were able to rotate individually in comparison to the majority of the commercial particles that tend to agglomerate in the presence of a $\vec{B}$ especially at higher magnetic fields (as shown in figure 5.8), making it harder to get a good sample number of particles rotating.

Considering all of the colloids assessed at all the intensities of the $\vec{B}$, only $23 \%$ of commercial particles analysed rotated $360^{\circ}$ and individually at $1 \mathrm{~Hz}$ and $26 \%$ rotated at 5 $\mathrm{Hz}$; whereas $77 \%$ of ellipsoidal particles rotated at $1 \mathrm{~Hz}$, and $74 \%$ did it at $5 \mathrm{~Hz}$. Out of 10 commercial spherical particles assessed at $0.1 \mathrm{mT}$, only 4 turned $360^{\circ}$ at $1 \mathrm{~Hz}$, and none 

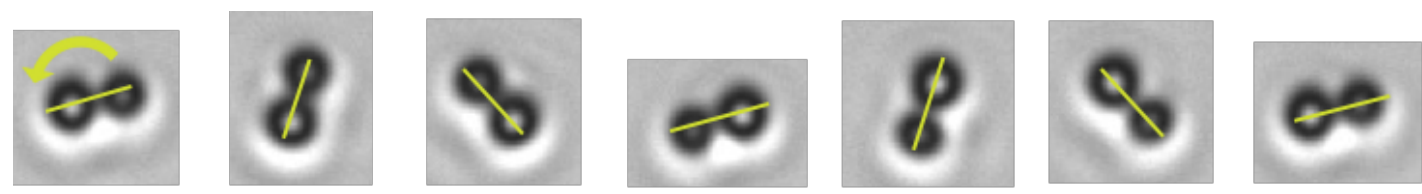

$$
t=0 \quad t=0.033 s \quad t=0.067 s \quad t=0.1 s \quad t=0.133 s \quad t=0.167 s \quad t=0.2 s
$$

Figure 5.8: Sequence of image frames acquired for two microparticles rotating together counter-clockwise (as indicated by the rotation arrow on the first frame) in a $20 \mathrm{mT}, 5 \mathrm{~Hz}$ rotating $\vec{B}$. Every third frame is shown. Yellow lines are the angle of the particle.
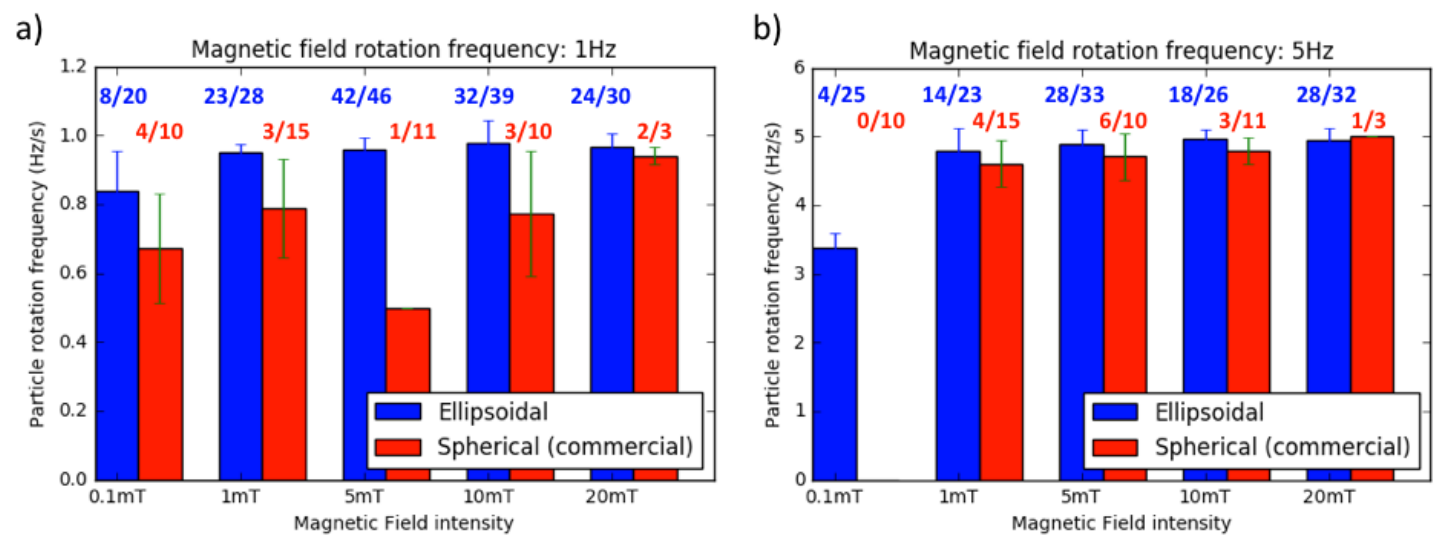

Figure 5.9: Angular velocities calculated for colloids when rotating at different intensities of the field with an applied $\vec{B}$ rotating at (a) $1 \mathrm{~Hz}$ and (b) $5 \mathrm{~Hz}$ respectively. These results do not take in account oscillations. Synthesised ellipsoidal particles are shown in blue and commercial spherical particles are shown in red. Fractions in the top of the bars represent the number of particles that completed at least one $360^{\circ}$ cycle divided by the total number of particles which were not agglomerated and considered in the analysis for each intensity of the field

at $5 \mathrm{~Hz}$.

\subsection{Conclusions}

Following a simple procedure based on the oil in water emulsion method we have been able to synthesise ellipsoidal polymeric magnetic microparticles based on a lattice of biocompatible PCL with embedded $\mathrm{Fe}_{3} \mathrm{O}_{4}$ nanowires. The microellipsoids are formed in the presence of a $\vec{B}$, hence the orientation of the nanowires inside the particle is not random but arising 
from the competing effect of nanowire-polymer interactions and nanowire-magnetic field interactions.

The result is a microparticle with ellipsoidal geometry, which shape anisotropy allows them to rotate at a given frequency of the external $\vec{B}$, as compared with spherical $\mathrm{Fe}_{3} \mathrm{O}_{4}$ microparticles.

It is important to mention that commercial spherical microparticles oscillate more than micorellipsoids at lower magnetic fields. This was quantified by obtaining the number of turns per second of all the particles that rotated at least $360^{\circ}$. The mean number of rotations per second resulted higher for microellipsoids in all cases $(0.1 / 1 \mathrm{mT}$ and $1 / 5 \mathrm{~Hz})$ than for commercial microparticles (Fig 5.6).

Our results show the superior rotation performance of the ellipsoidal particles in rotating magnetic fields in comparison to spherical commercial particles as it would be expected from their intrinsic shape anisotropy even though their magnetic material content is at least 20 times smaller than the commercial particles'.

The high symmetry of the ellipsoidal shape makes the particles amenable to simpler computational tracking and to theoretical calculations and modelling, which are key considerations for designing and fine-tuning future applications. The method of synthesis can be easily adapted with other polymeric matrices and particles. 


\section{Chapter 6}

\section{Conclusions and further work}

\subsection{Conclusions}

Polymers have been extensively used in biomedical applications because of their versatility of design. This thesis describes some applications of polymers in biomedicine, divided in two parts: the first one focuses on natural and composite hydrogel scaffolds for TE specially focusing on the importance of the mechanical design and characterisation and the second on magnetically responsive nanocomposites as force generators at the micron scale.

\subsubsection{Alginate microbead stability}

Chapter 3 mainly focuses on the mechanical characterisation of a couple of widely used materials for TE: Alginate 3.1 and Chitosan 3.2 .

The stability of alginate beads for modular TE was assessed in DMEM medium for a period of two weeks. Alginate is known to go through ionic exchange of $\mathrm{Ca}^{2+}$ from the bead's polymeric lattice with $\mathrm{Na}^{+}$ions on DMEM medium, which is widely used as a cell culture medium [188, 189]. This instability could alter the bead mechanically and make beads dissolve releasing the cells. Because of this, using AFM microindentation technique, 
we quantified the $\tilde{E}$ modulus using Hertz model and correlated it, at different time points, with the beads' swelling behaviour. We then compared freshly made beads with freeze dried beads.

Freeze drying is a process that could potentially be further employed in biomedical materials for preservation and storage, yet it is essential to understand how does the material changes mechanically due to this process.

Our study showed a higher $\tilde{E}$ modulus and a smaller diameter of freeze dried beads in comparison to freshly made beads. This is likely be due to higher porosity in the freeze drying process, increment of density and crosslinking of the material, that would result in longer ion exchange equilibrating time (from day 10). Future experiments, should involve the assessment of porosity change due to the freeze drying process.

Freshly made beads showed mechanical stability during the assessment period, yet freeze dried beads, showed a significant decrease after day 8 possibly due to the degradation which could potentially be solved by studies on freeze drying process optimisation. Our results can provide information on freshly made bead stability for microcarrier culture and cell encapsulation and the need of freeze drying optimisation.

\subsubsection{Chitosan hydrogel and nanocomposite}

In chapters 3 section 3.2 and 4 , we discuss hydrogels that are able to change their mechanical properties by the influence of a magnetic field. These polymers are proposed for TE applications since cellular differentiation can be directed via underlying scaffold stiffness [40, 52 54 and being able to change the substrate stiffness on demand could potentially be used to create different kinds of tissue in the same scaffold at different times and gives the opportunity to recreate native microstructure in healthy tissue. Furthermore, magnetically actuating the scaffold could improve the diffusion of nutrients, drugs and growth factors, or create vascularisation which has been a re-incurring challenge in the TE field. 
As described before in chapter 3, chitosan's mechano-magnetic coupling has never been reported before and is likely to expand and improve current applications (i.e. fabrication of drug delivery systems, tissue engineering scaffolds, water filtration) and inspire new uses in direct actuation and to improve the fabrication and performance of chitosan ferrogels and magnetic composites.

This coupling could be explained by magnetic hydrodynamic effects due to the presence of a magnetic field as well as an electrically conductive medium flowing through the pores of a piezoelectric polymer in the presence of a $\vec{B}$. The fluid could create a Lorentz force with the $\vec{B}$, affecting the mechanical properties of the hydrogel.

Because of this, our experiments indirectly support previous observations that indicate that chitosan presents piezoelectric behaviour [283]. Piezoelectricity refers to the accumulation of an electric charge, in response to applied mechanical stress. In our case, there is a $\vec{B}$ (which is always coupled with an electric field) that renders different mechanical properties due to a deformation and an electrolite flow given a magnetohydrodynamic effect.

The nanocomposite chitosan hydrogels, have magnetic nanowires embedded throughout the material. These magnetic nanowires might influence the produced Lorentz force, especially if the nanowires are oriented, enhancing the magneto-mechanical coupling.

There are still deficiencies in the proposed magneto-mechanical coupled materials, mainly, there is a need to homogenise the dispersion of nanowires and avoid cluster formation in addition to improve nanowire alignment by the use of stronger magnetic fields. Yet the overall behaviour could potentially have a positive impact on the TE field.

All of the local mechanical characterisations described in this thesis were performed at the micrometer scale since this is the scale of human cells ranging from $2-120 \mu \mathrm{m}$. By using an attached spherical indenter of 20 and $49.5 \mu \mathrm{m}$ to the AFM cantilever, we measured the $\tilde{E}$ modulus felt at the cellular scale. This is particularly important since polymeric 
materials are intrinsically inhomogeneous and special attention most be payed in order to design and synthesise materials for TE. This problem is specially enhanced in the case of nanocomposite materials where the nanostructures embedded within the polymer tend to agglomerate and form clusters.

In the case of the nanocomposite hydrogel synthesised in this thesis, we have superficial agglomerations of nanowires forming in the tens of micros scale. These might be the reason why local microindentation experiments resulted with such a big dispersion in $\tilde{E}$ modulus calculations presented in chapter 4

\subsubsection{Microellipsoids}

Lastly, in chapter 5, I describe another use of magnetically responsive nanocomposite, this time in the form of microellipsoids.

Magnetically responsive microellipsoids could be used in TE applications in for instance targeted modular TE. Furthermore, rotating microellipsoids could potentially be the bottom-up approach of rotational bioreactors widely used in the field.

These microellipsoids present enhanced capabilities to follow rotating magnetic fields for force and torque generation assays. The procedure to synthesise these ellipsoids is simple and could easily be modified to be used with other polymers.

Our results show the superior rotation performance of the ellipsoidal particles rotating at low magnetic fields ( 0.1 and $1 \mathrm{mT})$, although comparable to spherical commercial particles at higher fields, despite the magnetic content of the microellipsoids being at least 20 times less. Synthesised microellipsoides were also less prone to agglomeration when rotating at any intensity of the field, in contrast to the commercial particles that aggregated especially at higher intensities of the field.

Furthermore, commercial particles were more prone to oscillate at low $\vec{B}$. The number of turns per second is much less that in the case of the ellipsoidal ones. 
The idea and fabrication protocol have been patented since the microparticles are sold for several biomedical applications. The capacity to rotate to higher frequencies, using lower magnetic fields with simpler computational tracking and to theoretical calculations makes them attractive for widening some experimental borderlines in many applications, such as magnetic tweezers, hence commercially desirable.

\subsection{Further work}

\subsection{1 $\quad \mathrm{Fe}_{3} \mathrm{O}_{4}$ nanowires}

$\mathrm{Fe}_{3} \mathrm{O}_{4}$ magnetic nanowires were successfully synthesised and characterised as described in section 4.2.1. AFM, SEM, TEM and EDX microscopy were performed on them to characterise their shape, size and composition. AFM image 4.3 (b) and (d) shows a large dispersion of sizes in the nanowires from $\mu m$ to hundreds of $\mathrm{nm}$.

Figure 5.3 (b) shows one microparticle synthesised with $\mathrm{Fe}_{3} \mathrm{O}_{4}$ nanowires embedded inside, under a magnetic field as described in the materials and methods. It is evident the presence of spherical nanostructures from approximately $20 \mathrm{~nm}$ to $300 \mathrm{~nm}$ in diameter. Figures 4.4 and 6.1 show the EDX analysis of an area comprising only nanowires and one which contains a spherical structure of the order of approx. $150 \mathrm{~nm}$ respectively in diameter. Both images confirm the presence of $\mathrm{Fe}$ in the structure because of peaks at 0.7174 and $6.3967 \mathrm{keV}$.

This indicates an inefficient synthesis and that the magnetic field used during the synthesis is not strong enough as reported by Wang et al [140]. They demonstrate that by increasing the magnetic field intensity, the ratio of nanowires increased in comparison to the formation of porous nanostructures and squared particles.

The performance of both of the magnetic responsive nanocomposites described in this thesis: the microparticles (chapter 5) and the hydrogels for TE (chapter 4 could be en- 


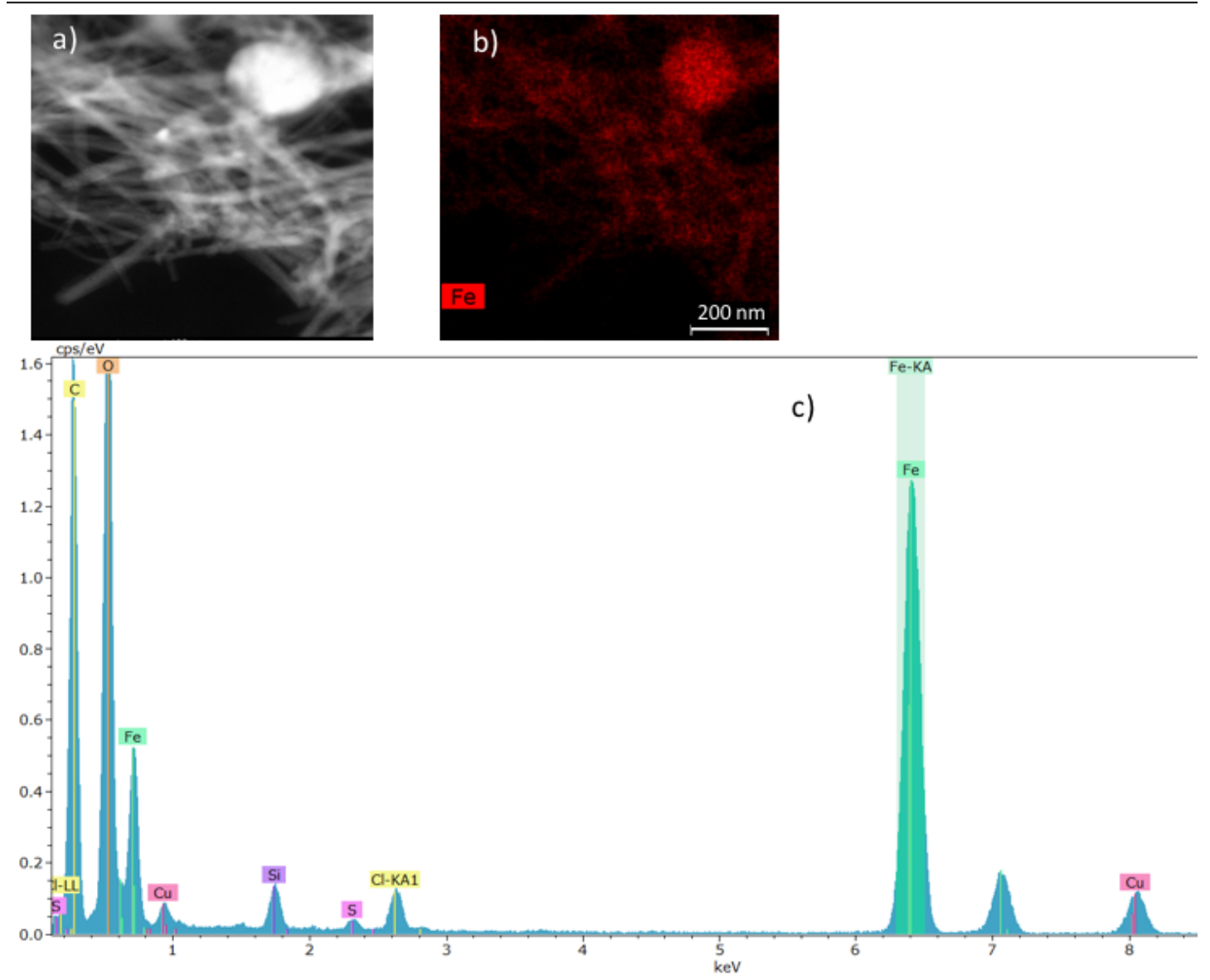

Figure 6.1: $\mathrm{Fe}_{3} \mathrm{O}_{4}$ nanostructures embedded within the polymeric PCL lattice showing the EDX analysis area (a), the Fe (red) content (b) and (c) the EDX analysis confirming $\mathrm{Fe}(0.7174$ and $6.3967 \mathrm{keV})$. 
hanced if these particles were not present in the nanocomposites. Future experiments should focus on the synthesis of perfectly monodispersed nanowires or the purification of nanowires from other nanostructures.

Furthermore, the synthesis reaction presented in chapter 4 has been reported to result in superparamagnetic nanowires. The EDX results presented above show the presence of Oxygen and Iron but SQUID magnetic characterisation is still necessary to characterise the magnetisation of the nanowires.

SQUID magnetometry is used to measure extremely subtle magnetic fields based on superconducting loops. By obtaining the magnetic susceptibility (proportionality constant that indicates the magnetisation response of a material subjected to a $\vec{B}$ ) as a function of temperature (ZFC-FC curves) and the magnetisation as a function of the applied $\vec{B}(\mathrm{M} / \mathrm{H}$ isotherms), we can assess the remanence and coercitivity of the nanowires, expecting no remanence and low coercitivity which are intrinsic characteristics of superparamagnets.

\subsubsection{Chitosan hydrogel scaffolds and nanocomposites}

Fig. (c, d) and (e-g) show the scaffolds synthesised in $\vec{B} 45^{\circ}$ and horizontal, respectively. Especially in $45^{\circ}$ (c, d), there is a clear direction of alignment (highlighted with white arrows) of clusters of nanowires (highlighted with red arrows) in the surface of the scaffold. (g) shows a big cluster of nanowires in the surface which explains the even higher inhomogeneities in the measured mechanical properties of nanocomposites.

Despite an apparent superficial nanowire alignment, further TEM experiments described in chapter 5, using similar intensities of the $\vec{B}$ to orient $\mathrm{Fe}_{3} \mathrm{O}_{4}$ nanowires inside microellipsoid instead of inside chitosan hydrogels, show nanowire cluster formation (figure 5.3 b). This may be due to competing polymer crosslinking forces that overcome nanowire$\vec{B}$ orienting forces. These behaviour is most likely to be similar in the case of the scaffolds, since forces created during the crystallisation of the water present in the scaffold before 
freeze drying, might overcome $\vec{B}$ induced forces on the nanowire orientation. In order to have a better nanowire alignment, more homogeneous and stronger magnetic fields should be used in both composite scaffolds and microellipsoids.

Despite the already discussed enhancement of inhomogeneity effects due to clusters of nanowires in the scaffold surface, there is an intrinsic effect observed in chitosan-only scaffolds due to the porous materials and as shown in figure 3.7 (a), where indentations on different positions in the polymer without a $\vec{B}$ vary from $2 \mathrm{kPa}$ (position 9 ), to as high as $62 \mathrm{kPa}$ (position 5). This big dispersion makes it hard to give an overall or mean value of $\Delta E^{*}$ because of a $\vec{B}$, yet changes are still visible at each position (Fig. 3.7 a) which does not happen for the PDMS control.

Another important aspect that would be important to work further on, is the change in porosity due to the presence of a $\vec{B}$. This could have implications in nutrient transport in scaffolds for TE.

Furthermore, an interesting behaviour of the hydrogels to assess is magnetostriction (a change in shape or dimensions during magnetization). This on account of observed expansion or contraction of the piezo when in contact with the sample and the application of a $\vec{B}$. Magnetostriction could broaden chitosan scaffolds or nanocomposites applications in fields as drug delivery.

Despite the interesting characteristics of these magneto-mechanical materials, there are still deficiencies on their design. Mainly, there is a need to homogenise the dispersion of nanowires and avoid cluster formation in addition to improve nanowire alignment by the use of stronger magnetic fields. Yet the overall behaviour could potentially have a positive impact on the TE field. 


\subsubsection{Microparticles}

One of the main problems regarding these described microellipsoids is the lack on orientation of the nanowires inside the bead.

Several factors influence the need of stronger $\vec{B}$, such as the viscosity of the oil phase where the nanowires are immersed in, as well as the competing crosslinking/hardening forces that might be stronger than the field and disrupt the orientation. Lastly, the centrifugal forces created by the emulsification process (vortexing) to form the microparticles might have an influence on the spherical nanowire clusters observed in figure 5.3 (b) and (c). Each microellipsoid might be a bigger cluster of smaller oil droplets agglomerating before the gelling of the agar. The fabrication of the microellipsoids by other methods such as 3D printing might reduce the formation of these clusters and enhance nanowire alignment as well as render monodisperse particle.

Furthermore, higher magnetic fields could be used during microparticle hardening and by optimising the crosslinking velocity of the polymer, wires could be oriented in a way that enhances even more the magnetic anisotropy already observed. 


\section{Appendix A}

\section{Medium charge and Lorentz force}

In order to assess the charges from the medium needed to change the mechanical properties of chitosan, we assume to have a change accelerated due to the normal force applied by the AFM cantilever that generates a Lorentz force. There is a change in the force necessary to obtain the same displacement of the material in a force vs indentation curve ( $\mathrm{F}$ vs $\delta$ ) in the presence and absence of the $\vec{B}$ ( $F_{M}$ and $F_{N M}$, respectively).

$$
\begin{gathered}
\Delta F=F_{M}-F_{N M} \neq 0 \\
F_{M}-F_{L}=F_{N M}
\end{gathered}
$$

where $F_{L}$ is the Lorentz force.

In order to estimate the Lorentz force, we use the following equation as a first approximation that does not take into account the effect of water, which is also conductive

$$
\overrightarrow{F_{L}}=q(\vec{v} \times \vec{B})
$$

where $\mathrm{q}$ is a charge moving at a velocity $\vec{v}$. 
If we assume a homogeneous $\vec{B}$,

$$
\overrightarrow{F_{L}} \hat{z}=q\left(v_{x} B_{y}-v_{y} B_{x}\right) \hat{z}
$$

If we also assume that the velocity of the charges, accelerated by the normal force applied to the hydrogel, will be smaller than the velocity of the indentation, then $\left(v_{x} B_{y}-\right.$ $\left.v_{y} B_{x}\right) \leq v_{\text {indentation }}\left(B_{y}-B_{x}\right)$ and

$$
\leq q 2 V_{\text {indent }} B_{\max }
$$

Solving this with a F vs $\delta$ curve obtained experimentally,

$$
q \geq \frac{\Delta F}{v_{i} B_{M}} \geq 0.017 C
$$

which corresponds to a number of charges $(\mathrm{N})$ :

$$
N=\frac{q}{e} \simeq 10^{17}
$$

where e is the elementary electronic charge and also corresponds to a number of moles $(n)$ :

$$
n=\frac{N}{N_{A}} \approx 10^{-6} \mathrm{~mol}
$$

In order to compare this theoretical value to the ion content in the medium we used, we calculated the amount of ions in $1 \mathrm{xPBS}$ pH $7.4(0.138 \mathrm{M} \mathrm{NaCl}, 0.0027 \mathrm{M} \mathrm{KCl}, 0.010 \mathrm{M}$ $\left.\mathrm{Na}_{2} \mathrm{HPO}_{4}, 0.0018 \mathrm{M} \mathrm{KH} \mathrm{PO}_{4}\right)$.

First, we calculate $\mathrm{H}_{3} \mathrm{O}^{+}$and $\mathrm{OH}^{-}$ions that result in a $\mathrm{pH}=7.4$ 


$$
\begin{array}{r}
p H=-\log _{10}\left[\mathrm{H}_{3} \mathrm{O}^{+}\right] \\
{\left[\mathrm{H}_{3} \mathrm{O}^{+}\right]=3.98 \times 10^{-8} M} \\
p O H=-\log _{10}\left[\mathrm{OH}^{-}\right] \\
{\left[\mathrm{OH}^{-}\right]=2.51 \times 10^{-7} M}
\end{array}
$$

Then, if we consider that the solute dissociates in the form:

$$
X_{A} Y_{B} \rightarrow X_{A}^{\alpha+}+Y_{B}^{\beta-}
$$

we can obtain the ion to solute ratio:

$$
\frac{\text { ion }}{\text { solute }}=\frac{X_{A}^{\alpha+}[\mathrm{mol}]}{X Y[\mathrm{~mol}]}
$$

In order to find ion molarity $(\mathrm{M})$ :

$$
M_{X_{A}^{\alpha+}}=M_{X Y}\left(\frac{X_{A}^{\alpha+}}{\text { solute }}\right)
$$

Solving for all the solutes in $1 x P B S$ :

- For $\mathrm{NaCl} \rightarrow \mathrm{Na}^{+}+\mathrm{Cl}^{-}[0.138 \mathrm{M}]$

$$
\begin{gathered}
\frac{\mathrm{Na}^{+}}{\text {solute }}=\frac{\mathrm{Cl}^{-}}{\text {solute }}=\frac{1 m o l \mathrm{Na}^{+}}{1 m o l \mathrm{Cl}^{-}} \\
M_{\mathrm{Na}^{+}}=M_{\mathrm{Cl}^{-}}=M_{N a C l}\left(\frac{1 m o l \mathrm{Na}^{+}}{1 m o l \mathrm{NaCl}}\right)=138 m M
\end{gathered}
$$


- For $\mathrm{KCl} \rightarrow \mathrm{K}^{+}+\mathrm{Cl}^{-}[0.0027 \mathrm{M}]$

$$
M_{\mathrm{K}^{+}}=M_{\mathrm{Cl}^{-}}=2.7 m M
$$

- For $\mathrm{Na}_{2} \mathrm{HPO}_{4} \rightarrow 2 \mathrm{Na}^{+}+\mathrm{HPO}_{4}{ }^{2-}[10 \mathrm{mM}]$

$$
\frac{\mathrm{Na}^{+}}{\text {solute }}=\frac{2 m o l \mathrm{Na}^{+}}{1 m o l \mathrm{Na}_{2} \mathrm{HPO}_{4}}=20 m M
$$

and

$$
\frac{\mathrm{HPO}_{4}{ }^{2-}}{\text { solute }}=\frac{1 \mathrm{~mol} \mathrm{HPO}_{4}{ }^{2-}}{1 m o l \mathrm{Na}_{2} \mathrm{HPO}_{4}}=10 \mathrm{mM}
$$

- For $\mathrm{KH}_{2} \mathrm{PO}_{4} \rightarrow \mathrm{K}^{+}+\mathrm{H}_{2} \mathrm{PO}_{4}^{-}[0.0018 \mathrm{M}]$

$$
M_{\mathrm{K}^{+}}=M_{\mathrm{H}_{2} \mathrm{PO}_{4}}=1.8 m M
$$

If we assume the volume displaced by the AFM probe due to the indentation to be in the order of $1 \mathrm{~mm}^{3}$, the moles can be calculated as:

$$
n=M\left(\frac{k m o l}{m^{3}}\right) V\left(m^{3}\right)
$$

For each type of ions present in the medium we obtain

$n_{\mathrm{OH}^{-}}=2.51 \times 10^{-7} \cdot 10^{3} \cdot 10^{-9} \propto 10^{-13} \mathrm{~mol}$

Similarly,

$$
\begin{aligned}
& n_{\mathrm{H}_{3} \mathrm{O}^{+}} \propto 10^{-14} \mathrm{~mol} \\
& n_{\mathrm{Na}^{+}} \propto 10^{-7} \mathrm{~mol} \\
& n_{\mathrm{Cl}^{-}} \propto 10^{-7} \mathrm{~mol} \\
& n_{\mathrm{K}^{+}} \propto 10^{-9} \mathrm{~mol} \\
& n_{\mathrm{HPO}_{4}{ }^{2-}} \propto 10^{-8} \mathrm{~mol}
\end{aligned}
$$


$n_{\mathrm{H}_{2} \mathrm{PO}_{4}-} \propto 10^{-9} \mathrm{~mol}$

If we consider that

$$
N=n N_{A}
$$

we can calculate the number of charges in the medium.

$$
\begin{aligned}
& N_{\mathrm{OH}^{-}}=10^{-13} \cdot 10^{23} \propto 10^{10} \\
& N_{\mathrm{H}_{3} \mathrm{O}^{+}} \propto 1^{9} \\
& N_{\mathrm{Na}^{+}} \propto 10^{16} \\
& N_{\mathrm{Cl}^{-}} \propto 10^{16} \\
& N_{\mathrm{K}^{+}} \propto 10^{14} \\
& N_{\mathrm{HPO}_{4}{ }^{2-}} \propto 10^{15} \\
& N_{\mathrm{H}_{2} \mathrm{PO}_{4}} \propto 10^{14}
\end{aligned}
$$

The total ion concentration is given by the sum of the contributions, resulting in the order of $10^{16}$.

The concentration of ions present in the used 1xPBS $\left(N_{\text {total }} \propto 10^{16}\right)$ is only one order of magnitude smaller compared to the calculated for the Lorentz force (equation A.5) $\left(N_{L}=10^{17}\right)$ yet, the presented calculation is not complete, since it is not considering the water present in the medium and the chitosan polarisation effect that is not easy to determine without further experiments. All of these components are likely to result in a higher number of charges present in the system. Despite this, the previous calculation is a very good approximation that could confirm a Lorentz Force resultant of 1xPBS medium flow as described in chapter 3

West et. al fabricated continuous flow microreactors where the integrated micropumping mechanism obviates the use of moving parts by acting locally on the electrolyte via a magnetohydrodynamic effect. They attained fluid velocities in the order of $300 \mu \mathrm{m} \mathrm{s}^{-1}$ when applying $1 \mathrm{kHz}$ magnetic fields from $10.8-13.5 \mathrm{mT}$, a current of $156 \mathrm{~mA}$ and using 
a $500 \mathrm{mM} \mathrm{KCl}$ electrolyte 254 $10^{17}$ ion concentration (calculated as described above considering $1 \mathrm{~mm}^{3}$ volume), as the concentration calculated for the 1XPBS electrolyte used in our experimental setup. The application of a voltage in West microreactors, will enhance the magnetohydrodynamic effect compared to our set up, yet it gives us an insight on magnetohydrodynamic applications on microfluidics and ionic currents producing Lorentz forces. 


\section{Appendix B}

\section{Torque calculation for rotating microellipsoids}

Microellipsoids were synthesised as described in section 5.2.1 and their performance was assessed by a 3D magnetic manipulation system described in 5.2.3. Particles were subject to rotating magnetic fields $(0.1,1,5,10$ and $20 \mathrm{mT})$ at different frequencies $(1$ and $5 \mathrm{~Hz})$. The angular velocity was obtained from the videos analysed by image processing.

Torque of both, spherical commercial microparticles and synthesised microellipsoids were calculated with Equation B.1 describes the angular motion of a particle that experiences an external torque in solution:

$$
I \ddot{\varphi}=-C \dot{\varphi}+T_{m a g}+\sqrt{2 k_{B} T C} W(t) .
$$

Here, $I$ is the moment of inertia of the particle and $\ddot{\varphi}$ is the angular acceleration, $\dot{\varphi}$ is the angular velocity of the particle, this whole inertial term can be neglected micron-sized particles in solution and small Reynold's number in comparison to the viscous drag $-C \dot{\varphi}$. $\varphi$ is the orientation angle of the particle, $C$ is the rotational friction coefficient, $T_{\text {mag }}$ is the torque and the last term corresponds to the stochastic rotational Brownian fluctuations 
at temperature $T ; k_{B}$ is the Boltzmann's constant and $W(t)$ is a random variable with Gaussian distribution of zero mean and unity variance [284].

As a first approximation, the rotational Brownian fluctuations can be neglected because the time scale of the directed rotational motion of the microparticles is much faster than the time scale of Brownian diffusive motion. Therefore, from the balance of magnetic torque and viscous drag, the magnetic torque on the particles can be approximately calculated as $T=C \dot{\varphi}$. Considering that our micro-particles are prolate ellipsoids with semi-axes $b \approx c<a$ and that they rotate around the short axis $\mathrm{b}$, we can calculate the rotational friction coefficient C using the formula [285]:

$$
C=\frac{32 \pi}{3} \eta \frac{\left(a^{4}-b^{4}\right)}{\left(2 a^{2}-b^{2}\right) S_{\text {prolate }}-2 a},
$$

where $\eta$ is the dynamic viscosity of water $\left(\eta=8.90 x 10^{-4}\right.$ Pa.s $)$. For prolate ellipsoids the geometrical factor $S_{\text {prolate }}$ can be approximated as:

$$
\left.S_{\text {prolate }}=\frac{2}{\sqrt{a^{2}-b^{2}}} \ln \frac{a+\sqrt{a^{2}-b^{2}}}{b}\right) .
$$

For the commercial spherical microparticles, the magnetic torque was calculated using the rotational friction coefficient corresponding to a $C_{\text {sphere }}$ [285]:

$$
C_{\text {sphere }}=\pi 8 a^{3} \eta
$$

The magnetic torque was calculated for all of elliptical and spherical commercial microparticles that at rotated at least $360^{\circ}$ once. 


\section{B.0.1 Torque produced by weak magnetic fields}

The torque exerted on each particle was calculated for all the particles that turned at least $360^{\circ}$ (Fig. B.1) using the procedure described in the Materials and Methods section. The torque was normalised to the volume calculated for the spherical $\left(\frac{4}{3} \pi a^{3}\right)$ or prolate ellipsoidal $\left(\frac{4}{3} \pi a b^{2}\right)$ particles, in order to compare particles of different volumes. Fig. B.1 shows that the increment of the applied magnetic field intensity results in a greater torque being applied to the microparticles, making them rotate closer to the applied rotational frequency as also shown in Fig. 5.9. The exerted torque for the commercial particles than the ellipsoidal synthesised ones is similar if we consider the error bars in nearly all cases. Despite this, it is important to mention that commercial spherical microparticles oscillate more at lower magnetic fields. The calculated angular velocity and torque are a result of the quantification of the particle's rotation when it gives a $360^{\circ}$ turn, yet, the number of turns per second of ellipsoidal particles is significantly higher than for commercial microparticles.

The calculated rotational friction coefficient $\mathrm{C}$, resulted higher for the spherical microparticles $\left(9.21 \times 10^{-19} \pm 2.72 \times 10^{-19}\right.$ N.m.s $)$ than the microellipsoids $\left(7.22 \times 10^{-19} \pm\right.$ $6.23 \times 10^{-19}$ N.m.s) for similar sized particles.

It is important to note that the magnetic content of the synthesised ellipsoidal microparticles is at least 20 times smaller for the spherical commercial microparticles which present a $10-15 \%$ weight in volume ratio. 

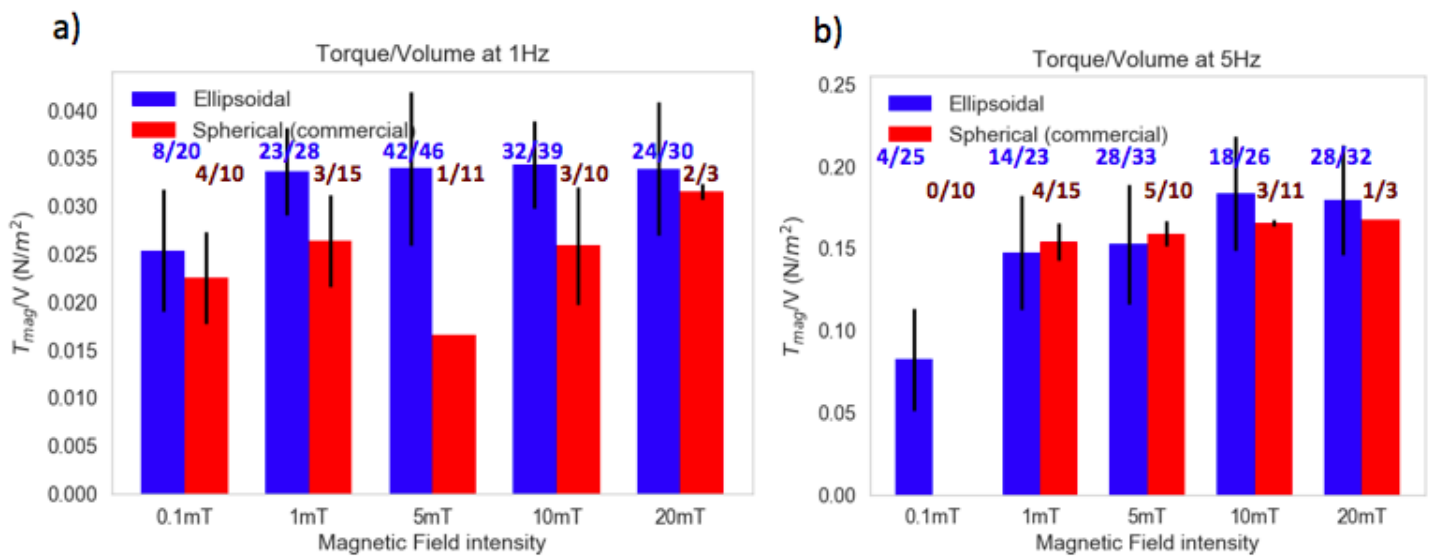

Figure B.1: Torque normalised by volume of all the particles that at least gave a $360^{\circ}$ turn, rotating at $1 \mathrm{~Hz}$ (left) and $5 \mathrm{~Hz}$ (right) and varying the intensity of the magnetic field. Ellipsoidal microparticles are plotted in blue and spherical in red with a line indicating the standard deviation 


\section{Bibliography}

[1] C. Mason, P. Dunnill, Regenerative Medicine 2008, 3, 1-5.

[2] C. A. Vacanti, Journal of Cellular and Molecular Medicine 2018, 10, 569-576.

[3] R. Lanza, R. Langer, J. Vacanti, Principles of tissue engineering, Elsevier Academic Press, 2007, p. 1307.

[4] A. Atala, S. B. Bauer, S. Soker, J. J. Yoo, A. B. Retik, Lancet Apr. 2006, 367, 1241-1246.

[5] The Department of Communication Karolinska University Hospital, First Successful Transplantation of a Synthetic Tissue Engineered Windpipe, 2011.

[6] M. E. Khosroshahi, Applications of biophotonics and nanobiomaterials in biomedical engineering, $\mathbf{2 0 1 7}$

[7] J. W. Nichol, A. Khademhosseini, Soft Matter 2009, 5, 1312-1319.

[8] J. K. Mouw, G. Ou, V. M. Weaver, Nature Publishing Group 2014, 15, 771.

[9] P. Birk D. E., Bruckner, The extracellular matrix : an overview, Springer, 2011, p. 425.

[10] Y. Kim, H. Ko, I. K. Kwon, I. K. Kwon, K. Shin, International neurourology journal May 2016, 20, S23-29.

[11] C. Bonnans, J. Chou, Z. Werb, Nature Reviews Molecular Cell Biology 2014, 15, 786-801.

[12] T. A. Wynn, T. R. Ramalingam, Mechanisms of fibrosis: Therapeutic translation for fibrotic disease, July 2012.

[13] P. Lu, V. M. Weaver, Z. Werb, The extracellular matrix: A dynamic niche in cancer progression, Feb. 2012.

[14] E. C. Chan, S.-M. Kuo, A. M. Kong, W. A. Morrison, G. J. Dusting, G. M. Mitchell, S. Y. Lim, G.-S. Liu, PLOS ONE Feb. 2016, 11, (Ed.: J.-Y. Lai), e0149799.

[15] H. Bramfeld, G. Sabra, V. Centis, P. Vermette, Current Medicinal Chemistry Nov. 2010, 17, $3944-3967$.

[16] M. Lovett, K. Lee, A. Edwards, D. L. Kaplan, Tissue engineering. Part B Reviews Sept. 2009, 15, 353-70.

[17] X. Xiao, W. Wang, D. Liu, H. Zhang, P. Gao, L. Geng, Y. Yuan, J. Lu, Z. Wang, Scientific Reports Mar. 2015, 5, 9409 .

[18] H. Miyoshi in Smart Materials for Tissue Engineering: Fundamental Principles, 2017, pp. 1-24.

[19] D. F. Williams, Biomaterials 2008, 29, 2941-2953.

[20] W. Elshahawy, Advances in Ceramics Electric and Magnetic Ceramics Bioceramics Ceramics and Environment 2011.

[21] H. H. Gustafson, D. Holt-Casper, D. W. Grainger, H. Ghandehari, Nano Today 2015, 10, 487-510.

[22] T. Kusaka, M. Nakayama, K. Nakamura, M. Ishimiya, E. Furusawa, PLoS ONE 2014, 9, 92634.

[23] H. L. Karlsson, J. Gustafsson, P. Cronholm, L. Möller, Toxicology Letters 2009, 188, $112-118$.

[24] M. M. Song, W. J. Song, H. Bi, J. Wang, W. L. Wu, J. Sun, M. Yu, Biomaterials 2010, 31, $1509-1517$.

[25] M. Safi, M. Yan, M.-A. Guedeau-Boudeville, H. Conjeaud, V. Garnier-Thibaud, N. Boggetto, A. Baeza-Squiban, F. Niedergang, D. Averbeck, J.-F. Berret, ACS Nano 2011, 5, 5354-5364.

[26] L. H. Reddy, J. L. Arias, J. Nicolas, P. Couvreur, American Chemical Society 2012, DOI 10.1021/cr300068p.

[27] R. G. Breuls, T. U. Jiya, T. H. Smit, The Open Orthopaedics Journal May 2008, 2, $103-109$.

[28] E. K. Paluch, C. M. Nelson, N. Biais, B. Fabry, J. Moeller, B. L. Pruitt, C. Wollnik, G. Kudryasheva, F. Rehfeldt, W. Federle, BioMed Central Biology 2015, 1-14.

[29] V. Vogel, M. P. Sheetz, Current Opinion in Cell Biology 2009, 21, 38-46. 
[30] A. L. Plant, K. Bhadriraju, T. A. Spurlin, J. T. Elliott, Biochimica et Biophysica Acta - Molecular Cell Research 2009, 1793, 893-902.

[31] K. A. Jansen, P. Atherton, C. Ballestrem, Seminars in Cell and Developmental Biology 2017, 71, 75-83.

[32] C. M. Lo, M. Wang, H. B.and Dembo, Y. L. Wang, Biophysical Journal 2000, 79, $144-152$.

[33] F. Grinnell, M. A. Carlson, F. Grinnell, M. Zhu, M. A. Carlson, J. M. Abrams, 1999, 619, 608-619.

[34] D. E. Ingber, Circulation Research 2002, 91, 877-887.

[35] E. Y. Lee, The Journal of Cell Biology 1984, 98, 146-155.

[36] M. Chiquet, Matrix Biology 1999, 18, 417-426.

[37] M. J. Paszek, V. M. Weaver, Journal of Mammary Gland Biology and Neoplasia 2004, 9, 325-342.

[38] L. K. Hansen, J. Wilhelm, J. T. Fassett, Current Topics in Developmental Biology 2005, 72, $205-236$.

[39] S. B. H. Timraz, R. Rezgui, S. M. Boularaoui, J. C. M. Teo, Procedia Engineering 2015, 110, $29-36$.

[40] A. J. Engler, S. Sen, H. L. Sweeney, D. E. Discher, Cell 2006, 126, 677-689.

[41] A. J. Engler, M. A. Griffin, S. Sen, C. G. Bönnemann, H. L. Sweeney, D. Discher, The Journal of Cell Biology 2004, 166, 345-348.

[42] L. J. Alberts B, Johnson A, Integrins, 4th, Garland Science, 2002.

[43] K. A. Jansen, D. M. Donato, H. E. Balcioglu, T. Schmidt, E. H. Danen, G. H. Koenderink, Biochimica et Biophysica Acta - Molecular Cell Research 2015, 1853, 3043-3052.

[44] OpenStax, Anatomy and Physiology, 2016, p. 128.

[45] K. S. Saladin, Anatomy \& physiology : the unity of form and function, McGraw-Hill, 2012.

[46] J. T. Finer, R. M. Simmons, J. A. Spudich, Nature Mar. 1994, 368, 113-9.

[47] S. Chanet, A. C. Martin, Progress in Molecular Biology and Translational Science 2014, 126, 317-352.

[48] L. K. Chin, Y. Xia, D. E. Discher, P. A. Janmey, Current Opinion in Chemical Engineering 2016, $11,77-84$.

[49] L. Kass, J. T. Erler, M. Dembo, V. M. Weaver, International Journal of Biochemistry and Cell Biology 2007, 39, 1987-1994.

[50] E. L. Baker, J. Lu, D. Yu, R. T. Bonnecaze, M. H. Zaman, Biophysical Journal 2010, 99, $2048-2057$.

[51] V. W. Wong, M. T. Longaker, G. C. Gurtner, Seminars in Cell and Developmental Biology 2012, $23,981-986$.

[52] L. Ghasemi-Mobarakeh, World Journal of Stem Cells 2015, 7, 728.

[53] D. E. Discher, Science 2005, 310, 1139-1143.

[54] K. Saha, A. J. Keung, E. F. Irwin, Y. Li, L. Little, D. V. Schaffer, K. E. Healy, Biophysical Journal 2008, 95, 4426-4438.

[55] R. Parenteau-Bareil, R. Gauvin, F. Berthod, Materials 2010, 3, 1863-1887.

[56] L. Cen, W. Liu, L. Cui, W. Zhang, Y. Cao, Pediatric Research 2008, 63, 492-496.

[57] A. M. Ferreira, P. Gentile, V. Chiono, G. Ciardelli, Acta Biomaterialia 2012, 8, 3191-3200.

[58] B. An, Y. S. Lin, B. Brodsky, Advanced Drug Delivery Reviews 2016, 97, 69-84.

[59] M. Jorfi, E. J. Foster, Journal of Applied Polymer Science 2015, 132, 1-19.

[60] S. Saravanan, S. Nethala, S. Pattnaik, A. Tripathi, A. Moorthi, N. Selvamurugan, International Journal of Biological Macromolecules Aug. 2011, 49, 188-193.

[61] L. Fu, P. Zhou, S. Zhang, G. Yang, Materials Science and Engineering C July 2013, 33, $2995-3000$.

[62] R. A. Pérez, J. E. Won, J. C. Knowles, H. W. Kim, Advanced Drug Delivery Reviews 2013, 65, 471-496.

[63] E. M. Ahmed, Hydrogel: Preparation, characterization, and applications: A review, Mar. 2015.

[64] D. I. Rudyardjo, S. Wijayanto in Journal of Physics: Conference Series, Vol. 853, 2017.

[65] A. S. Hoffman, Advanced drug delivery reviews Sept. 2012, 43, 3-12.

[66] R. L. Reis, N. M. Neves, J. F. Mano, M. E. Gomes, A. P. Marques, H. S. Azevedo, Natural-Based Polymers for Biomedical Applications, 2008, pp. 27-31.

[67] J. A. Rowley, D. J. Mooney, Journal of Biomedical Materials Research 2002, 60, $217-223$. 
[68] P. R. Hari, T. Chandy, C. P. Sharma, Journal of Applied Polymer Science 1996, 59, 1795-1801.

[69] J. L. Wilson, T. C. Mcdevitt, Biotechnology and Bioengineering 2013, 110, 667-682.

[70] S. J. Bidarra, C. C. Barrias, P. L. Granja, Acta Biomaterialia 2014, 10, 1646-1662.

[71] S. Demiroglu, V. Singaravelu, Fiber Technology for Fiber-Reinforced Composites Jan. 2017, 277-297.

[72] M. Savioli Lopes, A. L. Jardini, R. Maciel Filho in Procedia Engineering, Vol. 42, Elsevier, Jan. 2012, pp. 14021413.

[73] D. J. Mooney, C. L. Mazzoni, C. Breuer, K. McNamara, D. Hern, J. P. Vacanti, R. Langer in The Biomaterials: Silver Jubilee Compendium, Vol. 17, 2, Elsevier, Jan. 2006, pp. 129-138.

[74] D. Mondal, M. Griffith, S. S. Venkatraman, International Journal of Polymeric Materials and Polymeric Biomaterials Mar. 2016, 65, 255-265.

[75] E. Malikmammadov, T. E. Tanir, A. Kiziltay, V. Hasirci, N. Hasirci, Journal of Biomaterials Science Polymer Edition 2018, 29, 863-893.

[76] S. C. Chen, M. H. Yang, T. W. Chung, T. S. Jhuang, J. D. Yang, K. C. Chen, W. J. Chen, Y. F. Huang, S. B. Jong, W. C. Tsai, P. C. Lin, Y. C. Tyan, BioMed Research International Jan. 2017, 2017, 1-8.

[77] T. Laemthong, H. H. Kim, K. Dunlap, C. Brocker, D. Barua, D. Forciniti, Y.-W. Huang, S. Barua, Nanotechnology Jan. 2017, 28, 045601.

[78] Y. Chi, S. Zhu, C. Wang, L. Zhou, L. Zhang, Z. Li, Y. Dai, Journal of drug targeting 2016, 24, 224-32.

[79] C. Romagnoli, R. Zonefrati, G. Galli, D. Puppi, A. Pirosa, F. Chiellini, F. S. Martelli, A. Tanini, M. L. Brandi, BioMed Research International Oct. 2015, 2015, 1-12.

[80] J. Lee, J. Kim, M. Bae, S. Park, D. Balikov, H.-j. Sung, H. Jeon, H. Park, S. Um, K. Lee, I. Kwon, Polymers Feb. 2016, 8, 49 .

[81] A. Prabhakar, A. P. Lynch, M. Ahearne, Artificial Organs Apr. 2016, 40, 376-384.

[82] M. Karimi, A. Ghasemi, P. Sahandi Zangabad, R. Rahighi, S. M. Moosavi Basri, H. Mirshekari, M. Amiri, Z. Shafaei Pishabad, A. Aslani, M. Bozorgomid, D. Ghosh, A. Beyzavi, A. Vaseghi, A. R. Aref, L. Haghani, S. Bahrami, M. R. Hamblin, Smart micro/nanoparticles in stimulus-responsive drug/gene delivery systems, Vol. 45, Royal Society of Chemistry, 2016, pp. 1457-1501.

[83] R. De Santis, F. Sarracino, F. Mollica, P. A. Netti, L. Ambrosio, L. Nicolais, Composites Science and Technology 2004, 64, 861-871.

[84] F. Mirahmadi, M. Tafazzoli-Shadpour, M. A. Shokrgozar, S. Bonakdar, Materials Science and Engineering C 2013 , 33, 4786-4794.

[85] S. Yodmuang, S. L. McNamara, A. B. Nover, B. B. Mandal, M. Agarwal, T. A. N. Kelly, P. H. G. Chao, C. Hung, D. L. Kaplan, G. Vunjak-Novakovic, Acta Biomaterialia 2015, 11, 27-36.

[86] J. Venkatesan, S. K. Kim, Journal of Biomedical Nanotechnology 2014, 10, 3124-3140.

[87] R. A. MacDonald, B. F. Laurenzi, G. Viswanathan, P. M. Ajayan, J. P. Stegemann, Journal of Biomedical Materials Research - Part A 2005, 74, 489-496.

[88] K. M. Koss, L. D. Unsworth, Acta Biomaterialia 2016, 44, 2-15.

[89] M. Yamato, C. Konno, M. Utsumi, A. Kikuchi, T. Okano, Biomaterials 2002, 23, 561-567.

[90] P. Gupta, S. Sharan, P. Roy, D. Lahiri, Carbon 2015, 95, 715-724.

[91] X. Zhao, J. Kim, C. A. Cezar, N. Huebsch, K. Lee, K. Bouhadir, D. J. Mooney, Proceedings of the National Academy of Sciences 2011, 108, 67-72.

[92] C. Tapeinos, E. K. Efthimiadou, N. Boukos, G. Kordas, Colloids and Surfaces B: Biointerfaces 2016, 148, 95-103.

[93] V. Sundaresan, J. U. Menon, M. Rahimi, K. T. Nguyen, A. S. Wadajkar, International Journal of Pharmaceutics 2014, 466, 1-7.

[94] E. K. Efthimiadou, C. Tapeinos, L. A. Tziveleka, N. Boukos, G. Kordas, Materials Science and Engineering C 2014, 37, 271-277.

[95] P. Sanpui, A. Murugadoss, P. V. Prasad, S. S. Ghosh, A. Chattopadhyay, International Journal of Food Microbiology 2008, 124, 142-146.

[96] N. N. Reddy, S. Ravindra, N. M. Reddy, V. Rajinikanth, K. M. Raju, V. S. Vallabhapurapu, Journal of Magnetism and Magnetic Materials Nov. 2015, 394, 237-244.

[97] F. Patolsky, G. Zheng, C. M. Lieber, Nanomedicine 2006, 1, 51-65. 
[98] O. Yassine, A. Zah, E. Q. Li, A. Alfadhel, J. E. Perez, Scientific reports 2015, 1-7.

[99] J. Mohapatra, A. Mitra, H. Tyagi, D. Bahadur, M. Aslam, Nanoscale 2015, 7, 9174-9184.

[100] R. M. Erb, J. J. Martin, R. Soheilian, C. Pan, J. R. Barber, Advanced Functional Materials 2016, 26, 3859-3880.

[101] E. J. Peterman, F. Gittes, C. F. Schmidt, Biophysical Journal 2003, 84, 1308-1316.

[102] F. Petersson, L. Åberg, A. M. Swärd-Nilsson, T. Laurell, Analytical Chemistry 2007, 79, 5117-5123.

[103] A. Bhowmick, N. Pramanik, T. Mitra, A. Gnanamani, M. Das, P. P. Kundu, New J. Chem. 2017, 41, $190-197$.

[104] X. Y. Zhang, Y. Xue, Y. Zhang, Bioelectromagnetics Jan. 2006, 27, 1-9.

[105] M. T. Santini, G. Rainaldi, A. Ferrante, P. L. Indovina, P. Vecchia, G. Donelli, Bioelectromagnetics July 2003, 24, 327-338.

[106] J. MacFadden, L. Kirschvink, D. S. Jones, B. J., Magnetite Biomineralization and Magnetoreception in Organisms: A New Biomagnetism.

[107] M. Vahak, Ceramics Nano-Glass Ceramics: Processing, Properties and Applications, 2015.

[108] M. Molina, M. Asadian-Birjand, J. Balach, J. Bergueiro, E. Miceli, M. Calderón, Chemical Society Reviews 2015, 44, 6161-6186.

[109] S. M. Mirvakili, I. W. Hunter, Advanced Materials 2018, 30, 1-28.

[110] A. Candiani, A. Argyros, S. G. Leon-Saval, R. Lwin, S. Selleri, S. Pissadakis, Applied Physics Letters 2014, 104, DOI $10.1063 / 1.4869129$

[111] T. Hoare, B. P. Timko, J. Santamaria, G. F. Goya, S. Irusta, S. Lau, C. F. Stefanescu, D. Lin, R. Langer, D. S. Kohane, Nano Letters 2011, 11, 1395-1400.

[112] S. Palagi, P. Fischer, Nature Reviews Materials 2018, 3, 1-12.

[113] M. Khoo, C. Liu, Proceedings of the 22nd Annual International Conference of the IEEE Engineering in Medicine and Biology Society 2000, 3, 4-7.

[114] N.-T. Nguyen, X. Huang, T. K. Chuan, Journal of Fluids Engineering 2002, 124, 384.

[115] R. G. Thomas, A. R. Unnithan, M. J. Moon, S. P. Surendran, T. Batgerel, C. H. Park, C. S. Kim, Y. Y. Jeong, International Journal of Biological Macromolecules 2018, 110, 465-471.

[116] E. Amstad, J. Kohlbrecher, E. Müller, T. Schweizer, M. Textor, E. Reimhult, Nano Letters 2011, 11, 1664-1670.

[117] K. Zhang, Y. Fan, N. Dunne, X. Li, Regenerative Biomaterials Mar. 2018, 5, 115-124.

[118] Q. L. Loh, C. Choong, Tissue Engineering Part B: Reviews 2013, 19, 485-502.

[119] V. Karageorgiou, D. Kaplan, Biomaterials 2005, 26, 5474-5491.

[120] G. Lu, G. Q. Lu, Z. M. Xiao, Journal of Porous Materials 1999, 6, 359-368.

[121] N. Annabi, J. W. Nichol, X. Zhong, C. Ji, S. Koshy, A. Khademhosseini, F. Dehghani, Tissue Engineering Part B: Reviews Aug. 2010, 16, 371-383.

[122] J. Lannutti, D. Reneker, T. Ma, D. Tomasko, D. F. Farson, Materials Science \& Engineering C-Biomimetic and Supramolecular Systems 2007, 27, 504.

[123] Labconco, 2010, DOI 10.1017/CB09781107415324.004

[124] F. Khan, M. Tanaka, International Journal of Molecular Sciences 2018, 19, 1-14.

[125] B. L. Strand, A. E. Coron, G. Skjak-Braek, Stem cells translational medicine Apr. 2017, 6, 1053-1058.

[126] B. M. Leung, M. V. Sefton, Annals of Biomedical Engineering Nov. 2007, 35, 2039-2049.

[127] S. Lin, L. Gu, Materials 2015, 8, 551-560.

[128] A. Menyhárd, P. Suba, Z. László, H. M. Fekete, O. Mester, Z. Horváth, G. Vörös, J. Varga, J. Móczó, Express Polymer Letters 2015, 9, 308-320.

[129] Z. Gong, S. E. Szczesny, S. R. Caliari, E. E. Charrier, O. Chaudhuri, X. Cao, Y. Lin, R. L. Mauck, P. A. Janmey, J. A. Burdick, V. B. Shenoy, Proceedings of the National Academy of Sciences Mar. 2018, 115, 201716620.

[130] E. H. Barriga, R. Mayor, Adjustable viscoelasticity allows for efficient collective cell migration, June 2018.

[131] F. D. Liu, N. Pishesha, Z. Poon, T. Kaushik, K. J. Van Vliet, ACS Biomaterials Science and Engineering 2017, 3, 3292-3306.

[132] M. Capurro, F. Barberis, Evaluating the mechanical properties of biomaterials, Woodhead Publishing Limited, 2014, pp. 270-323. 
[133] C. Wang, C. Cowen, Z. Zhang, C. Thomas, Chemical Engineering Science Dec. 2005, 60, 6649-6657.

[134] I. Chasiotis, Q. Chen, G. M. Odegard, T. S. Gates, Experimental Mechanics 2005, 45, 507-516.

[135] D. M. Ebenstein, L. A. Pruitt, Nano Today Aug. 2006, 1, 26-33.

[136] T. G. Kuznetsova, M. N. Starodubtseva, N. I. Yegorenkov, S. A. Chizhik, R. I. Zhdanov, Micron Dec. 2007, 38, $824-833$

[137] Y. Zhu, Z. Dong, U. C. Wejinya, S. Jin, K. Ye, Journal of Biomechanics Sept. 2011, 44, $2356-2361$.

[138] S. Tomkoria, K. Masuda, J. Mao, Proceedings of the Institution of Mechanical Engineers Part H: Journal of Engineering in Medicine 2007, 221, 467-473.

[139] B. Janković, J. Pelipenko, M. Škarabot, I. Muševič, J. Kristl, International Journal of Pharmaceutics Oct. 2013, 455, 338-347.

[140] B. J. Wang, Q. Chen, C. Zeng, Advanced Materials 2004, 2002-2005.

[141] S. Chowdhury, V. Thomas, D. Dean, S. A. Catledge, Y. K. Vohra, Journal of Nanoscience and Nanotechnology Nov. 2005, 5, 1816-1820.

[142] T. K. L. Meyvis, B. G. Stubbe, M. J. Van Steenbergen, W. E. Hennink, S. C. De Smedt, J. Demeester, International journal of pharmaceutics Sept. 2002, 244, 163-8.

[143] X. Shi, J. L. Hudson, P. P. Spicer, J. M. Tour, R. Krishnamoorti, A. G. Mikos, Nanotechnology July 2005, 16, S531-S538.

[144] W. Zhu, V. C. Mow, T. J. Koob, D. R. Eyre, Journal of Orthopaedic Research Nov. 1993, 11, 771-781.

[145] W. J. Polacheck, C. S. Chen, Nature methods 2016, 13, 415-423.

[146] A. H. B. D. Vries, B. E. Krenn, R. V. Driel, J. S. Kanger, Biophysical Journal 2005, 88, 2137-2144.

[147] C. Haber, D. Wirtz, Review of Scientific Instruments 2000, 4561.

[148] J. Ali, U. K. Cheang, Y. Liu, H. Kim, L. Rogowski, S. Sheckman, P. Patel, W. Sun, M. J. Kim, J. Ali, U. K. Cheang, Y. Liu, H. Kim, L. Rogowski, AIP Advances 2016.

[149] T. Xu, J. Yu, X. Yan, H. Choi, L. Zhang, Micromachines 2015, 6, 1346-1364.

[150] A. Rida, M. A. M. Gijs, Analytical Chemistry 2004, 76, 6239-6246.

[151] C. Wilhelm, J. Browaeys, A. Ponton, J. Bacri, Physical Review Letters 2003, DOI 10.1103/PhysRevE.67.011504

[152] J. Lipfert, J. W. J. Kerssemakers, T. Jager, Nature methods 2010, 7, DOI 10.1038/nmeth.1520

[153] Z. Ye, M. Sitti, Lab on a chip 2014, 2177-2182.

[154] R. S. M. Rikken, R. J. M. Nolte, J. C. Maan, J. C. M. V. Hest, D. A. Wilson, P. C. M. Christianen, Soft Matter 2014, 10, 1295-1308.

[155] M. M. Van Oene, L. E. Dickinson, B. Cross, F. Pedaci, J. Lipfert, N. H. Dekker, Scientific Reports 2017 , 7, 1-12.

[156] Lluis M. Martinez, Biomagnetic Separation: Thinking Bigger, Part II, 2016.

[157] M. Puig-De-Morales, M. Grabulosa, J. Alcaraz, J. Mullol, G. N. Maksym, J. J. Fredberg, D. Navajas, Journal of Applied Physiology 2001, 91, 1152-1159.

[158] N. Pamme, Lab on a chip 2006, 1, DOI 10.1039/b513005k

[159] S.-w. Cao, Y.-j. Zhu, M.-y. Ma, L. Li, L. Zhang, Journal of Physical Chemistry 2008, 112, 1851-1856.

[160] C. Lin, H. Zhang, L. Li, Y. Yang, X. Liu, B. Xu, Journal of Applied Polymer Science 2015, DOI 10.1002/app.42617

[161] J. Faraudo, J. S. Andreu, C. Calero, J. Camacho, Advanced Functional Materials 2016, 26 , $3837-3858$.

[162] V. J. Morris, a. R. Kirby, a. P. Gunning, Atomic force microscopy for biologists, 2010.

[163] Y. M. Efremov, W.-H. Wang, S. D. Hardy, R. L. Geahlen, A. Raman, Scientific reports May $2017,7,1541$.

[164] L. M. Rebelo, J. S. De Sousa, J. Mendes Filho, M. Radmacher, Nanotechnology 2013, 24, DOI 10.1088/09574484/24/5/055102

[165] P. Attard, Measurement and interpretation of elastic and viscoelastic properties with the atomic force microscope, 2007.

[166] H.-J. Butt, B. Cappella, M. Kappl, Surface Science Reports 2005, 59, 1-152.

[167] P. P. Database, ADHESION CONTACT MECHANICS, 2015.

[168] L. D. Landau, E. M. Lifshitz, Course of Theoretical Physics 1986, 3, 109. 
[169] J. J. Abbott, M. P. Kummer, A. M. Hirt, B. J. Nelson, IEEE TRANSACTIONS ON ROBOTICS 2007, 23, 12471252 .

[170] M. Kopycinska-Müller, R. H. Geiss, D. C. Hurley, Ultramicroscopy 2006, 106, 466-474.

[171] U. D. Schwarz, O. Zwörner, P. Köster, R. Wiesendanger, Physical Review B Sept. 1997, 56, 6987-6996.

[172] X. Li, Y. H. An, Y.-D. Wu, Y. C. Song, Y. J. Chao, C.-H. Chien, Journal of Biomedical Materials Research Part B: Applied Biomaterials 2005, 83, 340-344.

[173] I. Titushkin, M. Cho, Biophysical Journal 2007, 93, 3693-3702.

[174] Andrei, DC, Briscoe, BJ, Luckham, PF, Williams, DR, J. Chim. Phys. 1996, 93, 960-976.

[175] D. Weitz, H. Wyss, R. Larsen, GIT laboratory journal Europe 2007, 11, 68-70.

[176] D. McMullan, Scanning Dec. 2006, 17, 175-185.

[177] A. Bogner, P. H. Jouneau, G. Thollet, D. Basset, C. Gauthier, Micron 2007, 38, 390-401.

[178] K. Havancsák, High-Resolution Scanning Electron Microscopy.

[179] A. World, Transmission electron microscope(TEM) - Principle of TEM.

[180] P. B. Malafaya, G. A. Silva, R. L. Reis, Advanced Drug Delivery Reviews 2007, 59, 207-233.

[181] J. Sun, H. Tan, Materials 2013, 1285-1309.

[182] T. R. Hoare, D. S. Kohane, Polymer 2008, 49, 1993-2007.

[183] G. Orive, R. Maria Hernández, A. Rodrï£·ï · i£·ï · guez Gascón, R. Calafiore, T. M. Swi Chang, P. D. Vos, G. Hortelano, D. Hunkeler, I. Lacï£· i $£ \cdot i £ \cdot i j \cdot k$, J. Luis Pedraz, Trends in Biotechnology Feb. 2004, 22, 87-92.

[184] P. J. Morris, Trends in Biotechnology 1996, 14, 163-167.

[185] S. N. A. Rahim, A. Sulaiman, F. Hamzah, K. H. K. Hamid, M. N. M. Rodhi, M. Musa, N. A. Edama, Procedia Engineering 2013, 68, 411-417.

[186] K. Narra, U. Dhanalekshmi, G. Rangaraj, D. Raja, C. S. Kumar, P. N. Reddy, A. B. Mandal, Iranian Journal of Pharmaceutical Research 2012, 11, 715-721.

[187] K. Y. Lee, D. J. Mooney, Progress in polymer science Jan. 2012, 37, 106-126.

[188] L. F. Tay, L. K. Khoh, C. S. Loh, E. Khor, Biotechnology and bioengineering 1993, 42, 449-54.

[189] M. D. Darrabie, W. F. Kendall, E. C. Opara, Journal of microencapsulation Feb. 2006, 23, 29-37.

[190] R. M. Kleinberger, N. a. D. Burke, K. Dalnoki-Veress, H. D. H. Stöver, Materials Science and Engineering C 2013, 33, 4295-4304.

[191] F. Guilak, D. M. Cohen, B. T. Estes, J. M. Gimble, W. Liedtke, C. S. Chen, Cell Stem Cell 2009, 5, 17-26.

[192] S. Bajpai, S. Sharma, Reactive and Functional Polymers May 2004, 59, 129-140.

[193] P. Yuan, Y. Jia, L. Zhang, J. Zhang, W. Hu, C. Wang, Journal of Wuhan University of Technology-Mater. Sci. Ed. July 2012, 27, 669-674.

[194] G. Pasparakis, N. Bouropoulos, International Journal of Pharmaceutics Oct. 2006, 323, 34-42.

[195] E.-S. Chan, T.-K. Lim, W.-P. Voo, R. Pogaku, B. T. Tey, Z. Zhang, Particuology June 2011, 9, $228-234$.

[196] A. Gaumann, M. Laudes, B. Jacob, R. Pommersheim, C. Laue, W. Vogt, J. Schrezenmeir, 2000, 21, 1911-1917.

[197] R. Gugerli, E. Cantana, C. Heinzen, U. V. Stockar, I. W. Marison, Journal of Microencapsulation 2002, 19, 571590.

[198] C. D. Markert, X. Guo, A. Skardal, Z. Wang, S. Bharadwaj, Y. Zhang, K. Bonin, M. Guthold, Journal of the Mechanical Behavior of Biomedical Materials 2013, 27, 115-127.

[199] M. Cavo, M. Fato, L. Peñuela, F. Beltrame, R. Raiteri, S. Scaglione, Scientific Reports 2016, 6, 35367.

[200] N. Helfricht, E. Doblhofer, V. Bieber, P. Lommes, V. Sieber, T. Scheibel, G. Papastavrou, Soft Matter 2017, 13, $578-589$.

[201] N. Patel, D. Lalwani, S. Gollmer, E. Injeti, Y. Sari, J. Nesamony, Progress in Biomaterials 2016, 5, $117-133$.

[202] M. Lekka, D. Sainz-Serp, A. J. Kulik, C. Wandrey, Langmuir : the ACS journal of surfaces and colloids 2004, 20, 9968-9977.

[203] J. L. Drury, D. J. Mooney, Biomaterials Nov. 2003, 24, 4337-4351.

[204] L. Gasperini, D. Maniglio, C. Migliaresi, Journal of Bioactive and Compatible Polymers Aug. 2013, $28,413-425$. 
[205] R. Freshney in Culture of Animal Cells: A Manual of Basic Technique and Specialized Applications, 2011.

[206] M. Szczypka, D. Splan, H. Woolls, H. Brandwein, BioProcess International 2014, 12, 54-64.

[207] A. K.-L. Chen, S. Reuveny, S. K. W. Oh, Biotechnology Advances 2013, 31, 1032-1046.

[208] E. Markvicheva, C. Grandfils in Fundamentals of Cell Immobilisation Biotechnology, 2004, pp. 141-161.

[209] D. Guo, J. Li, G. Xie, Y. Wang, J. Luo, Langmuir 2014, 30, 7206-7212.

[210] C. S. D. Lee, H. R. Moyer, R. a. Gittens I., J. K. Williams, A. L. Boskey, B. D. Boyan, Z. Schwartz, Biomaterials 2010, 31, 4926-4934.

[211] M. Serra, C. Correia, R. Malpique, C. Brito, J. Jensen, P. Bjorquist, M. J. T. Carrondo, P. M. Alves, PLoS ONE Aug. 2011, 6, (Ed.: C. Chan), e23212.

[212] C. Lechanteur, Journal of Stem Cell Research \& Therapy 2014, 04, 1-11.

[213] J. Barley, Basic Principles of Freeze Drying.

[214] G. Liu, Z. Hu, R. Guan, Y. Zhao, H. Zhang, B. Zhang, Korean Journal of Chemical Engineering 2016, 33, 31413148 .

[215] J.-H. Choi, H. Y. Lee, J.-C. Kim, Polymers and Polymer Composites 2013, 21, 449-456.

[216] J. L. Hutter, J. Bechhoefer, Review of Scientific Instruments 1993, 64, 1868-1873.

[217] N. Gavara, Scientific Reports 2016, 6, -13.

[218] B. S. Kim, Y. H. Choi, C. Y. Choi, B. G. Kim, Biotechnology Techniques 1992, 6, 347-352.

[219] G. T. Grant, E. R. Morris, D. a. Rees, P. J. C. Smith, D. Thom, FEBS Letters 1973, 32, 195-198.

[220] B. L. Strand, O. Gåserød, B. Kulseng, T. Espevik, G. Skjåk-Baek, Journal of microencapsulation 2002, 19, 615-30.

[221] Ä. A. Mørch, I. Donati, B. L. Strand, G. Skja, 2006, 1471-1480.

[222] E. A. Nunamaker, E. K. Purcell, D. R. Kipke, Journal of Biomedical Materials Research - Part A Dec. 2007, 83, $1128-1137$.

[223] E. Dintwa, E. Tijskens, H. Ramon, Granular Matter 2008, 10, 209-221.

[224] S. Kim, S. Lee, J. Lee, B. J. Nelson, L. Zhang, H. Choi, Scientific Reports 2016, 6, 1-9.

[225] D. Ivaneyko, V. Toshchevikov, M. Saphiannikova, G. Heinrich, Soft Matter 2014, 10, 2213-2225.

[226] S. Gil, J. F. Mano, Biomaterials Science 2014, 2, 812-818.

[227] J. Thevenot, H. Oliveira, O. Sandre, S. Lecommandoux, Chemical Society Reviews 2013, 42, 7099-7116.

[228] P. Sorlier, C. Viton, A. Domard, Biomacromolecules 2002, 2, 1336-1342.

[229] J. Desbrieres, E. Guibal, Polymer International 2018, 67, 7-14.

[230] I. Aranaz, N. Acosta, C. Civera, B. Elorza, J. Mingo, C. Castro, M. Gandia, A. H. Caballero, Polymers 2018, 10, DOI $10.3390 /$ polym10020213

[231] H. X. Wang, J. Qan, F. Y. Ding, Journal of Agricultural and Food Chemistry 2018, 66, 395-413.

[232] M. Malerba, R. Cerana, Polymers 2018, 10, DOI 10.3390/polym10020118

[233] R. Singh, K. Shitiz, A. Singh, International Wound Journal 2017, 14, 1276-1289.

[234] A. Oryan, S. Sahvieh, Effectiveness of chitosan scaffold in skin, bone and cartilage healing, Nov. 2017.

[235] G. L. Huang, Y. Liu, L. Chen, Drug Delivery 2017, 24, 108-113.

[236] Y. Yang, S. P. Wang, Y. T. Wang, X. H. Wang, Q. Wang, M. W. Chen, Biotechnology Advances 2014, 32, 13011316.

[237] K. Balagangadharan, S. Dhivya, N. Selvamurugan, International Journal of Biological Macromolecules 2017, 104, $1372-1382$.

[238] H. Liu, C. Y. Wang, C. Li, Y. G. Qin, Z. H. Wang, F. Yang, Z. H. Li, J. C. Wang, Rsc Advances 2018, 8, 7533-7549.

[239] T. K. Giri, A. Thakur, A. Alexander, Ajazuddin, H. Badwaik, D. K. Tripathi, Acta Pharmaceutica Sinica B Oct. 2012, 2, 439-449.

[240] F. Croisier, C. Jérôme, European Polymer Journal 2013, 49, 780-792.

[241] R. A. Muzzarelli, Genipin-crosslinked chitosan hydrogels as biomedical and pharmaceutical aids, May 2009.

[242] J. Jin, M. Song, D. J. Hourston, Biomacromolecules 2004, 5, 162-168. 
[243] L. C. Lins, G. C. Bazzo, P. L. Barreto, A. T. Pires, Journal of the Brazilian Chemical Society 2014, 25, $1462-1471$.

[244] M. Ahmad, K. Manzoor, S. Ikram, International Journal of Biological Macromolecules 2017, 105, 190-203.

[245] K. D. Yao, W. G. Liu, Z. Lin, X. H. Qiu, Polymer International 1999, 48, 794-798.

[246] D. Verma, K. S. Katti, D. R. Katti, B. Mohanty, Materials Science and Engineering C Apr. 2008, $28,399-405$.

[247] M. J. Moura, M. M. Figueiredo, M. H. Gil, Biomacromolecules 2007, 8, 3823-3829.

[248] A. Hänninen, S. Rajala, T. Salpavaara, M. Kellomäki, S. Tuukkanen, Procedia Engineering 2016, 168, 1176-1179.

[249] H. Alfvén, Existence of electromagnetic-hydrodynamic waves, Oct. 1942.

[250] L. D. Landau, L. P. Pitaevskii, E.M. Lifshitz, Electrodynamics of continuous media, Butterworth-Heinemann, 1984, p. 460.

[251] S. Qian, H. H. Bau, Mechanics Research Communications Jan. 2009, 36, 10-21.

[252] L. T. Cheah, I. Fritsch, S. J. Haswell, J. Greenman, Biotechnology and Bioengineering July 2012, 109, $1827-1834$.

[253] M. Zubair Akbar, M. Ashraf, M. Farooq Iqbal, K. Ali, AIP Advances Apr. 2016, 6, 045222.

[254] J. West, B. Karamata, B. Lillis, J. P. Gleeson, J. Alderman, J. K. Collins, W. Lane, A. Mathewson, H. Berney, Lab on a Chip Nov. 2002, 2, 224.

[255] J.-k. Xu, F.-f. Zhang, J.-j. Sun, J. Sheng, F. Wang, M. Sun, Molecules 2014, 19, 21506-21528.

[256] H.-P. Peng, R.-P. Liang, L. Zhang, J.-D. Qiu, Biosensors and Bioelectronics Apr. 2013, 42, 293-299.

[257] M. Baghayeri, E. Nazarzadeh Zare, M. Mansour Lakouraj, Biosensors \& bioelectronics May 2014, 55, 259-65.

[258] Y. Liu, M. Yuan, L. Qiao, R. Guo, Biosensors and Bioelectronics Feb. 2014, 52, 391-396.

[259] C. Sun, O. Veiseh, J. Gunn, C. Fang, S. Hansen, D. Lee, R. Sze, R. G. Ellenbogen, J. Olson, M. Zhang, Small Mar. 2008, 4, 372-379.

[260] R. Singh, S. Singh, P. Parihar, V. P. Singh, S. M. Prasad, Ecotoxicology and Environmental Safety 2015, 112, $247-270$

[261] M. Sadat, R. Patel, J. Sookoor, S. L. Bud'ko, R. C. Ewing, J. Zhang, H. Xu, Y. Wang, G. M. Pauletti, D. B. Mast, D. Shi, Materials Science and Engineering: $C$ Sept. 2014, 42, 52-63.

[262] S. Kumari, R. P. Singh, International Journal of Biological Macromolecules July 2012, 51, 76-82.

[263] A. Alfadhel, B. Li, A. Zaher, O. Yassine, J. Kosel, Lab Chip Oct. 2014, 14, 4362-4369.

[264] A. Alfadhel, J. Kosel, Advanced Materials Dec. 2015, 27, 7888-7892.

[265] W.-S. Lin, H.-M. Lin, H.-H. Chen, Y.-K. Hwu, Y.-J. Chiou, Journal of Nanomaterials 2013, 2013, 1-6.

[266] M. F. Contreras, R. Sougrat, A. Zaher, T. Ravasi, J. Kosel, International journal of nanomedicine 2015, 10, 214153.

[267] S. Lian, E. Wang, Z. Kang, Y. Bai, L. Gao, M. Jiang, C. Hu, L. Xu, Solid State Communications 2004, 129, $485-490$

[268] S. C. Bhatia, N. Ravi, Biomacromolecules 2000, 1, 413-417.

[269] W.-l. Chiang, C.-j. Ke, Z.-x. Liao, S.-y. Chen, F.-r. Chen, C.-Y. Tsai, Y. Xia, H.-W. Sung, Small 2012, 8, 35843588 .

[270] O. Ergeneman, S. Gervasoni, B. Ozkale, P. Fatio, V. J. Cadarso, B. J. Nelson, Nanoscale 2014, 6, DOI 10.1039/ C3NR06442E

[271] M. Suter, O. Ergeneman, J. Zürcher, C. Moitzi, S. Pané, T. Rudin, S. E. Pratsinis, B. J. Nelson, C. Hierold, Sensors and Actuators B: Chemical 2011, 156, 433-443.

[272] A. Butykai, A. Orbán, V. Kocsis, D. Szaller, S. Bordács, E. Tátrai-Szekeres, L. F. Kiss, A. Bóta, B. G. Vértessy, T. Zelles, I. Kézsmárki, Scientific Reports 2013, 3, 1-10.

[273] S. Tottori, L. Zhang, F. Qiu, K. K. Krawczyk, A. Franco-Obregõn, B. J. Nelson, Advanced Materials 2012, 24, 811-816.

[274] C. Sun, H. Hassanisaber, R. Yu, S. Ma, S. S. Verbridge, C. Lu, Scientific reports 2016, 1-9.

[275] M. A. M. Gijs, Microfluid Nanoflui 2004, 1, 22-40.

[276] V. Reenen, D. Jong, D. Toonder, M. Prins, Lab on a chip 2014, 12, DOI 10.1039/C3LC51454D

[277] M. Puig-de-morales, M. Grabulosa, J. Alcaraz, J. Mullol, G. N. Maksym, J. J. Fredberg, D. Navajas, M. Grabulosa, J. Al-, J. Mullol, G. N. Maksym, J. Jeffrey, D. Navajas, Journal of Applied Physiology 2001, 91, 1152-1159. 
[278] N. Wang, J. P. Butler, D. E. Ingber, Science 1993, 260, 1124-1128.

[279] H.-s. Lin, J. R. Carey, Journal of Nanoscience and Nanotechnology 2014, 14, 363-377.

[280] A. Vila, V. C. Martins, A. Chícharo, C. Rodriguez-abreu, A. C. Fernandes, F. A. Cardoso, S. Cardoso, J. Rivas, P. Freitas, IEEE TRANSACTIONS ON MAGNETICS 2014, 50, 18-21.

[281] L. Clime, B. L. Drogoff, T. Veres, IEEE TRANSACTIONS ON MAGNETICS 2007, 43, $2929-2931$.

[282] J. Kim, S. E. Chung, S.-e. Choi, H. Lee, J. Kim, S. Kwon, Nature Materials 2011, 10, 747-752.

[283] E. Praveen, S. Murugan, K. Jayakumar, RSC Advances July 2017, 7, 35490-35495.

[284] J. Nguyen, S. Contera, I. Llorente Garcia, RSC Advances 2016, 6, 46643-46653.

[285] F. Perrin, Le journal de physique et le radium 1934, 7. 